Research Article

\title{
Multipopulation Ensemble Particle Swarm Optimizer for Engineering Design Problems
}

\author{
Ziang Liu $\mathbb{D}^{1}$ and Tatsushi Nishi $\mathbb{D}^{2}$ \\ ${ }^{1}$ Graduate School of Engineering Science, Osaka University, 1-3 Machikaneyama-Cho, Toyonaka 560-8531, Japan \\ ${ }^{2}$ Graduate School of Natural Science and Technology, Okayama University, 3-1-1 Tsushima-Naka, Kita-Ku, \\ Okayama 700-8530, Japan
}

Correspondence should be addressed to Ziang Liu; ziang@inulab.sys.es.osaka-u.ac.jp and Tatsushi Nishi; nishi.tatsushi@okayama-u.ac.jp

Received 3 August 2020; Revised 19 September 2020; Accepted 6 October 2020; Published 11 November 2020

Academic Editor: Peter Dabnichki

Copyright (C) 2020 Ziang Liu and Tatsushi Nishi. This is an open access article distributed under the Creative Commons Attribution License, which permits unrestricted use, distribution, and reproduction in any medium, provided the original work is properly cited.

Particle swarm optimization (PSO) is an efficient optimization algorithm and has been applied to solve various real-world problems. However, the performance of PSO on a specific problem highly depends on the velocity updating strategy. For a realworld engineering problem, the function landscapes are usually very complex and problem-specific knowledge is sometimes unavailable. To respond to this challenge, we propose a multipopulation ensemble particle swarm optimizer (MPEPSO). The proposed algorithm consists of three existing efficient and simple PSO searching strategies. The particles are divided into four subpopulations including three indicator subpopulations and one reward subpopulation. Particles in the three indicator subpopulations update their velocities by different strategies. During every learning period, the improved function values of the three strategies are recorded. At the end of a learning period, the reward subpopulation is allocated to the best-performed strategy. Therefore, the appropriate PSO searching strategy can have more computational expense. The performance of MPEPSO is evaluated by the CEC 2014 test suite and compared with six other efficient PSO variants. These results suggest that MPEPSO ranks the first among these algorithms. Moreover, MPEPSO is applied to solve four engineering design problems. The results show the advantages of MPEPSO. The MATLAB source codes of MPEPSO are available at https:/github.com/zi-ang-liu/MPEPSO.

\section{Introduction}

Particle swarm optimization (PSO), proposed by Kennedy and Eberhart [1], is a stochastic and population-based optimization algorithm. PSO is one of the most popular optimization algorithms since its fast convergence rate, easy implementation, and effective performance for many optimization problems [2]. PSO has been successfully applied in various fields, such as engineering design problems [3-6], feature selection problems [7-9], scheduling problems [10-12], and supply chain management [13-15]. To further improve the performance of PSO, many PSO variants have been proposed by adapting the control parameters [16-18], designing new search strategies [19-22], introducing new population topologies $[23,24]$, and hybridizing PSO with other algorithms [25-28], etc.
The performance of PSO on a specific problem highly depends on the velocity updating strategy [29]. For example, PSO with time-varying acceleration coefficients (PSOTAVC) [30] performs well on unimodal problems, while performs poorly on multimodal problems. On the other hand, the distance-based locally informed PSO (LIPS) [31] is specially designed for solving multimodal problems. Furthermore, it has been illustrated that using different PSO searching strategies for a specific problem at different stages may improve the performance of the algorithm [29]. Therefore, it is important to find suitable searching strategies for a certain problem at different stages.

However, real-world engineering problems are usually very complex and problem-specific knowledge is sometimes unavailable. As a result, it is hard to analytically find the appropriate strategy for these problems. One way to select a 
suitable PSO searching strategy for real-world engineering problems is to adopt a trial-and-error approach. However, this approach is usually very time-consuming. On the other hand, by incorporating several different PSO searching strategies into one algorithm, and let the algorithm dynamically change the searching strategies during the computation process may enable us to solve various problems efficiently.

Motivated by this idea, a multipopulation ensemble particle swarm optimizer (MPEPSO) is proposed to solve real-world engineering problems when problem-specific knowledge is unavailable. Recently, the ensemble strategy is getting popular and many novel PSO algorithms have been proposed such as heterogeneous PSO (HPSO) [32], selfadaptive learning-based PSO (SL-PSO) [29], Heterogeneous comprehensive learning PSO (HCLPSO) [33], and ensemble PSO (EPSO) [34]. These previously proposed algorithms $[29,32-34]$ have been tested on classical benchmark functions or the CEC 2005 test suite. However, few of them have been applied to solve engineering design problems. In HPSO, the particles randomly change their search strategies. In HCLPSO, the proportion of strategies keeps the same. SLPSO uses a probability model to update the adoption probability of a strategy, and EPSO uses a success rate to update the adoption probability of a strategy. Instead of using these methods, the novelty of this paper is that MPEPSO dynamically assigns the particles in the reward subpopulation to the different PSO strategies based on their performances.

The purpose of this paper is to propose a novel PSO algorithm based on the ensemble approach and examine the performance of this algorithm in solving four well-known engineering design problems including the tension/compression spring design problem, the pressure vessel design problem, the cantilever beam design problem, and the gear train design problem. The tension/compression spring design problem is a nonlinear programming problem and the pressure vessel design problem is a mixed-integer nonlinear programming problem. The presence of integer variables along with continuous variables often occurs in engineering design and that adds to the complexity of the optimization problem [35].

In this paper, three existing simple and efficient PSO search strategies, linearly decreasing inertia weight PSO (LDWPSO) [16], unified PSO (UPSO) [36], and comprehensive learning PSO (CLPSO) [20], are incorporated into the proposed MPEPSO algorithm. These three strategies are selected because their searching behaviors are different yet complementary, so that they can support each other during the computational process. LDWPSO performs well on unimodal problems, but suffers from the premature problem and usually converge to the local optimum quickly. CLPSO has better performance on multimodal problems and is able to maintain the population diversity, while performs poorly on unimodal problems [34]. Different from the former two algorithms, a population topology is used in UPSO, so that the particles are guided by their neighbors. Also, UPSO has balanced exploration and exploitation ability, by combining the global PSO and local PSO [36]. In MPEPSO, the particles are divided into four subpopulations including three indicator subpopulations and one reward subpopulation. The fundamental idea of this ensemble method is similar to the approaches in $[37,38]$. The indicator subpopulations have a relatively small population size compared with the reward subpopulation. Particles in the three indicator subpopulations update their velocities by LDWPSO, UPSO, and CLPSO searching strategies, respectively. A certain number of iterations is defined as a learning period. MPEPSO keeps records of the improved function values by the three indicator subpopulations during the learning period. At the end of one learning period, the reward subpopulation is allocated to the PSO strategy that has the best performance during the learning period.

The performance of MPEPSO is examined on the CEC 2014 test suite on both 10-dimensional and 30-dimensional problems. Furthermore, MPEPSO is applied to solve four real-world engineering design problems. The results suggest that the proposed MPEPSO performs well in solving various problems by assembling the different features of these three algorithms.

This study makes several contributions to the current literature. First, a novel multipopulation ensemble particle swarm optimizer is proposed. This is the first time that the multipopulation ensemble strategy is used to develop a new PSO algorithm. Second, most previous studies of the ensemble approach-based PSO algorithms have focused on solving unconstrained benchmark problems, while our proposed algorithm is applied to solve four constrained engineering design problems. Third, the proposed algorithm is evaluated by comparing it with other algorithms on the CEC 2014 test suite. The mean, best, worst, and standard deviation values of the function error values are provided, and the Wilcoxon signed-rank test is conducted to determine the significance of the difference between the proposed algorithm and other algorithms.

The remaining part of the paper proceeds as follows: Section 2 gives a review of the related work. Section 3 presents a detailed introduction of MPEPSO. Computation results are reported in Section 4. Finally, Section 5 summarizes the conclusions.

\section{Related Work}

2.1. Particle Swarm Optimization. PSO is a population-based algorithm. In PSO, the position of a particle represents a solution for the problem. Particles update their velocities by social and cognitive experience. The velocity $V_{i}^{d}$ and position $X_{i}^{d}$ are usually updated by the following equations [2]:

$$
\begin{aligned}
V_{i}^{d}= & V_{i}^{d}+c_{1} \times \operatorname{rand}_{i}^{d} \times\left(\text { pbest }_{i}^{d}-X_{i}^{d}\right)+c_{2} \times \operatorname{rand}_{i}^{d} \\
& \times\left(g \text { best }^{d}-X_{i}^{d}\right), \\
X_{i}^{d}= & X_{i}^{d}+V_{i}^{d},
\end{aligned}
$$

where $i(i=1,2, \ldots, N)$ represents the index of particles, $N$ represents the population size, $d(d=1,2, \ldots, D)$ represents the dimension index, $D$ represents the dimensionality of the 
problem, $\operatorname{rand} 1_{i}^{d}$ and $\operatorname{rand} 2_{i}^{d}$ are two different random numbers within $[0,1], c_{1}$ and $c_{2}$ are the accelerate coefficients, pbest $t_{i}^{d}$ represents the personal best position of particle $i$ in dimension $d$, and $g$ best $^{d}$ represents the global best position of the swarm in dimension $d$.

Only the accelerate coefficients need to be tuned in PSO. $c_{1}$ and $c_{2}$ are the weights of the two directions, pbest and $g$ best. These two accelerate coefficients are set to 2 or 1.49445 in many studies [2]. The time-varying acceleration coefficients approach is another way to set these two parameters [30]. With a large $c_{1}$ and small $c_{2}$ at the early stage, the global exploration ability of particles is enhanced. By setting a small $c_{1}$ and large $c_{2}$ at the latter stage, the local exploitation ability of particles is improved.

2.2. Ensemble Approaches in PSO Algorithms. Ensemble approaches have been widely applied in population-based algorithms. See [39] for a detailed review of ensemble approaches in population-based algorithms. PSO algorithm is one of the popular population-based algorithms. Several researchers have also proposed the improved PSO variants based on the ensemble approach.

Wang et al. [29] incorporate four PSO searching strategies into their proposed algorithm. The execution probabilities of the four strategies are obtained by their proposed self-adaptive learning mechanism. Furthermore, their proposed algorithm has been applied to solve the economic load dispatch problem. The results indicate that this algorithm is able to solve complex large-scale economic load dispatch problem.

Lynn and Suganthan [33] divided all the particles into two subpopulations, an explorative subpopulation and an exploitative subpopulation. Particles in the explorative subpopulation are guided by the personal best experiences of particles in this subpopulation, while particles in the exploitative subpopulation are guided by the personal best experiences of all the particles. The experimental results indicate that their proposed algorithm performs well on the CEC 2005 benchmark functions.

Later, Lynn and Suganthan [34] incorporated five PSO searching strategies into their algorithm. The successful memory and the failure memory of each strategy are recorded during the learning period. The PSO strategy with a higher success rate is expected to have a higher adaptation probability. The computational results suggest that their proposed algorithm performs well on four types of benchmark functions in the CEC 2005 test suite.

The former studies that applied ensemble approaches in the PSO algorithm have shown that incorporating PSO searching strategies with different features into one algorithm may improve the performance of the PSO algorithm.

Several ensemble approaches have been used to develop new PSO variants. Wang et al. [29] used a probability model to control the probability of a strategy. Lynn and Suganthan [34] record the success rate of a strategy to control the adoption probability of a strategy. Different from the former studies, our proposed algorithm contains three indicator subpopulations. By evaluating the performance of the three subpopulations, the reward subpopulation is dynamically allocated to the good performed strategy. Also, few of the former proposed ensemble approach-based PSO variants have been applied to solve engineering design problems. To fill this gap, in this paper, the proposed algorithm is also applied to solve four real-world engineering design problems.

\section{Multipopulation Ensemble Particle Swarm Optimizer}

The main idea of MPEPSO is to incorporate PSO searching strategies with different features into one algorithm and dynamically allocate particles to the best-performed searching strategy. In this way, the advantaged PSO searching strategy can use more computational resources to improve the performance of the proposed algorithm.

Particles in MPEPSO are divided into three indicator subpopulations and one reward subpopulation. Each indicator subpopulation contains the same small number of particles, and particles in different indicator subpopulations update their velocity by different searching strategies. Indicator subpopulations are used to evaluate the performance of the corresponding searching strategy on a problem. The reward subpopulation is a group of particles that change their searching strategy periodically. The indicator subpopulations have a relatively small number of particles compared with the reward subpopulation. In this paper, particles in the three indicator subpopulations update their velocities by LDWPSO, UPSO, and CLPSO strategy, respectively. A learning period is defined as a fixed number of iterations. At the end of a learning period, the performance of each indicator subpopulation is evaluated to select the best-performed searching strategy, and then the reward subpopulation is allocated to the corresponding strategy. Therefore, the appropriate PSO searching strategy can have more particles to find a better solution.

Figure 1 is used as an example of a multipopulation in the proposed algorithm with twenty particles. Each indicator subpopulation has four particles and eight particles are assigned to the reward subpopulation. The particles in the reward subpopulation are dynamically allocated to the bestperformed indicator subpopulation.

3.1. PSO Variants. To enable MPEPSO to solve various problems, it is important to select the component strategies that have different capabilities. In this paper, three PSO searching strategies are incorporated in MPEPSO, including LDWPSO [16], UPSO [36], and CLPSO [20]. The reason that we select these three strategies is that their searching behaviors are different yet complementary. They can support each other during the computational process, by dynamically adopting these strategies. LDWPSO has a strong exploitation ability and it is very efficient in solving unimodal problems, while CLPSO is good at exploration and has high performance in solving multimodal problems. UPSO has a population topology that the particles are guided by their 


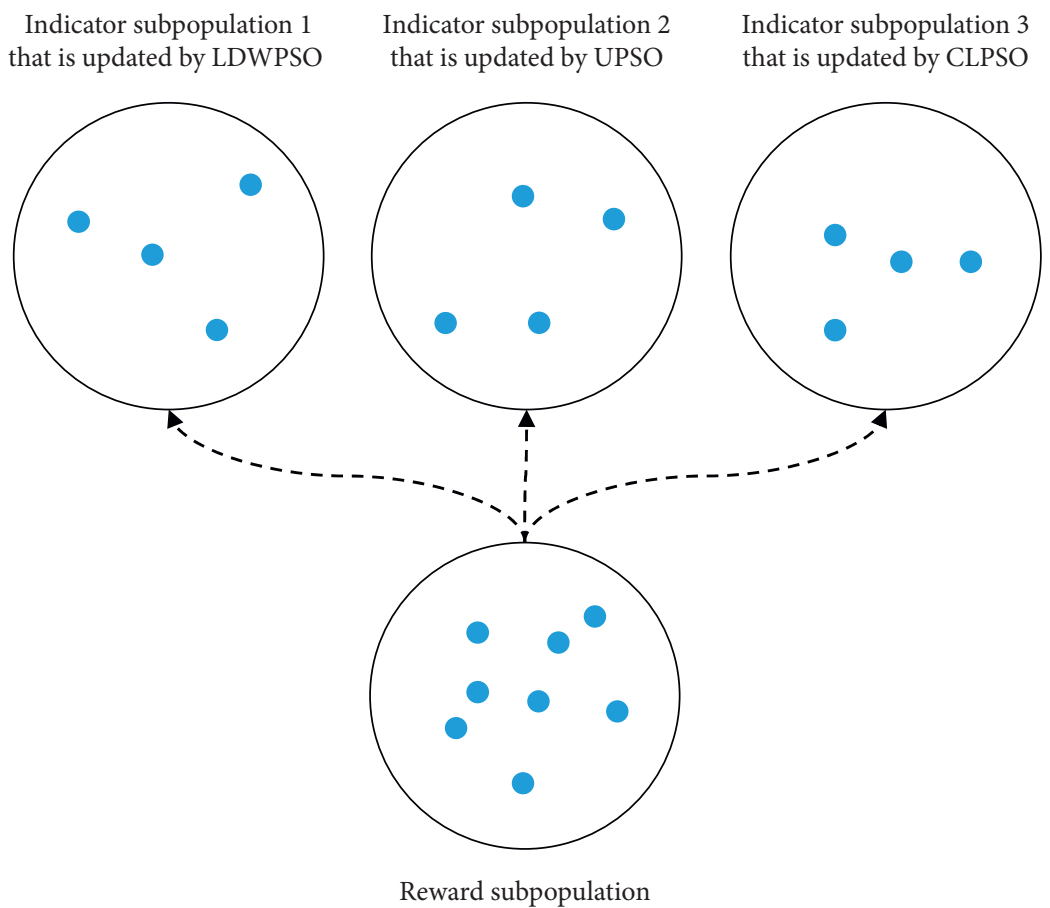

Figure 1: An example of multipopulation.

neighbors, and it combined the exploration ability of local PSO and the exploitation ability of global PSO. The velocity of a particle in UPSO is affected by both the local and global PSO variants.

3.1.1. Linearly Decreasing Inertia Weight PSO (LDWPSO). LDWPSO is one of the most famous PSO variants proposed by Shi and Eberhart [16]. The velocity $V_{i}^{d}$ in LDWPSO is updated as follows:

$$
\begin{aligned}
V_{i}^{d}= & \omega V_{i}^{d}+c_{1} \times \operatorname{rand}_{i}^{d} \times\left(\text { pbest }_{i}^{d}-X_{i}^{d}\right)+c_{2} \\
& \times \operatorname{rand}_{i}^{d} \times\left(\text { gest }^{d}-X_{i}^{d}\right) .
\end{aligned}
$$

By introducing the inertia weight $\omega$ into the original PSO algorithm, LDWPSO can improve the performance of the original PSO [2]. In this algorithm, $\omega$ is linearly decreased from $\omega_{\max }$ to $\omega_{\min }$ as follows:

$$
\omega=\omega_{\max }-\left(\omega_{\max }-\omega_{\min }\right) \frac{\mathrm{FEs}}{\mathrm{MaxFEs}},
$$

where FEs indicates the number of functions evaluations, MaxFEs indicates the maximum number of function evaluations. A large inertia weight is used for global search, and a small inertia weight is suitable for local search [20]. In many cases, $\omega$ is linearly decreased from 0.9 to 0.4 [16].

3.1.2. Unified Particle Swarm Optimization (UPSO). In the PSO algorithm, it is important to balance the exploration behavior and exploitation behavior of the particles. UPSO [36], proposed by Parsopoulos and Varhatis, has combined the local and global versions of the PSO algorithm to balance the exploration and exploitation ability of this algorithm.
In the local version of PSO, each particle only exchanges information with its neighbors. In this paper, the ring topology is adopted to decide the neighbors of a particle. In the ring topology, particle $(i-1)$ and particle $(i+1)$ are defined as the neighbors of particle $i$. In the global version of PSO, the whole population is the neighbors of particles.

The velocities of particles in the global and the local version PSO are shown as follows:

$$
\begin{aligned}
G_{i}^{d}= & X\left[V_{i}^{d}+c_{1} \times \operatorname{rand}_{i}^{d} \times\left(\text { pbest }_{i}^{d}-X_{i}^{d}\right)\right. \\
& \left.+c_{2} \times \operatorname{rand} 2_{i}^{d} \times\left(\text { gest }^{d}-X_{i}^{d}\right)\right], \\
L_{i}^{d}= & X\left[V_{i}^{d}+c_{1} \times \operatorname{rand}_{i}^{d} \times\left(\text { pest }_{i}^{d}-X_{i}^{d}\right)+c_{2} \times \operatorname{rand}_{i}^{d}\right. \\
& \left.\times\left(\text { nbest }_{i}^{d}-X_{i}^{d}\right)\right],
\end{aligned}
$$

where $\mathscr{X}$ represents the constriction factor, $n$ best $_{i}$ is the personal best position of the best neighborhood of $X_{i}$, velocity $G_{i}^{d}$ is obtained by the global version PSO, and velocity $L_{i}^{d}$ is obtained by the local version PSO. The constriction factor has been introduced to analyze the convergence behavior of the PSO algorithm, and the PSO algorithm with the constriction factor is a special case of the algorithm with inertia weight [40].

After obtaining the global velocity $G_{i}^{d}$ and the local velocity $L_{i}^{d}$ for each particle, these two directions can be unified to calculate the new velocity $U_{i}^{d}$ by the following equation:

$$
U_{i}^{d}=r_{i}^{d} \times u \times G_{i}^{d}+(1-u) \times L_{i}^{d}
$$

where

$$
r_{i}^{d} \sim N\left(\mu, \sigma^{2}\right)
$$


and $u$ represents the unification factor that balances the global and local components, $r_{i}^{d}$ follows a normal distribution. Finally, the position of the particle $i$ in $d$ dimension can be updated in the similar way to the original PSO algorithm as follows:

$$
X_{i}^{d}=X_{i}^{d}+U_{i}^{d}
$$

By unifying the local PSO and the global PSO, UPSO has combined its exploration and exploitation abilities [36].

\subsubsection{Comprehensive Learning Particle Swarm Optimizer} (CLPSO). CLPSO, designed by Liang et al. [20], is a popular and efficient PSO variant with a learning strategy that enables particles guided by the experience of the whole population. The velocity is updated as the following equation:

$$
V_{i}^{d}=\omega V_{i}^{d}+c_{1} \times \operatorname{rand}_{i}^{d} \times\left(\text { pbest }_{f i(d)}^{d}-X_{i}^{d}\right),
$$

where $f_{i}=\left[f_{i}(1), f_{i}(2), \ldots, f_{i}(D)\right]$ indicates which particles' pbest the particle $i$ should follow. Two particles are randomly selected from the population. The particle with better fitness will be used as $f_{i}$. See [20] for a detailed introduction. The decision that the particle $i$ learns from its own pbest or other's pbest depends on the learning probability $P c_{i}$. The previous study [20] indicates that the value of $P c_{i}$ affects the performance of CLPSO on multimodal problems. In CLPSO, each particle has a different value of $P c_{i}$, so that particles can have different levels of exploration and exploitation ability. The equation to obtain $P c_{i}$ is developed empirically. The learning probability $P c_{i}$ is calculated as follows:

$$
P c_{i}=a+b \times \frac{(\exp (10(i-1) / N-1)-1)}{(\exp (10)-1)},
$$

where $N$ represents the population size and $a$ and $b$ are used to tune the learning probability.

In the original PSO, particles are guided by the global best experience and their own personal best experience. However, CLPSO enables particles to learn from the personal best experience of all the particles, and the global best experience is not used. Comparing with the original PSO, particles in CLPSO are not easily be attracted to the global best experience and quickly converge to a local optimum. Therefore, this novel learning strategy keeps the diversity of the population [20]. Experimental results suggest that CLPSO has good performance in solving multimodal problems [20].

3.2. Proposed Algorithm. MPEPSO has one reward subpopulation pop $_{r}$ and three indicator subpopulations that represent by pop $_{h}$, where $h \in\{1,2,3\}$ is the index of the PSO searching strategy that corresponds to the LDWPSO, UPSO, and CLPSO. $\lambda_{h}$ is the proportion of pop $_{h}$ in the population. The value of $\lambda_{h}$ effects how many particles are in the indicator subpopulations. A large $\lambda_{h}$ makes fewer particles in the reward subpopulation, and the reward subpopulation will be less effective. On the other hand, a small $\lambda_{h}$ makes fewer particles in the indicator subpopulations, and it will be less accurate to evaluate the performance of each strategy. Empirically, we set $\lambda_{h}=0.1$ in this paper.

The population size of the indicator subpopulation $N_{h}$ is calculated as follows

$$
N_{h}=\left\lfloor N * \lambda_{h}\right\rfloor .
$$

At each iteration, $N_{h}$ particles are randomly selected from the total population and allocated to subpopulation pop $_{h}$. Particles in pop $h$ update their velocities by strategy $h$. Naturally, the number of particles in the reward subpopulation $N_{r}$ is obtained by

$$
N_{r}=N-\sum_{h=1,2,3} N_{h} .
$$

A learning period (LP) is defined as a certain number of iterations. During each learning period, if particle $i$ that uses strategy $h$ can produce a better solution comparing with its personal best position $p$ best $_{i}$, the strategy improvement $\Delta f_{h}$ will be recorded by

$$
\Delta f_{h}=\Delta f_{h}+F\left(\text { pbest }_{i}\right)-F\left(X_{i}\right), \quad i \in \text { pop }_{h} .
$$

At the end of the learning period, by comparing the value of $\Delta f_{h}$, the best-performed strategy will be selected and indexed by $k$ :

$$
k=\arg \left(\max _{h=1,2,3}\left(\frac{\Delta f_{h}}{\left\lfloor N * \lambda_{h}\right\rfloor}\right)\right) .
$$

The reward population is represented by pop $_{r}$. Particles in pop $_{r}$ will be allocated to pop $_{k}$ and update their velocities by the best-performed strategy $k$. By dynamically allocating the reward subpopulation to a good performed strategy at a different learning period, the appropriate PSO searching strategy will have more particles to find a better solution. Therefore, the performance of MPEPSO is expected to be enhanced. The learning period is used to tune the learning speed during the computation process. We empirically set the learning period at 10 in the proposed algorithm.

The pseudocode of the MPEPSO algorithm is described in Table 1. iter represents the number of iterations, $h$ is the index of strategies including LDWPSO, UPSO, and CLPSO, and $X_{\min }$ and $X_{\max }$ define the search range. rand is a $D$-dimensional vector with random numbers within $[0,1]$.

\section{Performance Evaluation}

4.1. Experimental Setting. The performance of MPEPSO is tested on the CEC 2014 benchmark functions [41, 42]. This is one of the most used test suites for evaluating the performance of evolutionary and swarm intelligence algorithms. This test suite includes 30 different types of unimodal, simple multimodal, hybrid, and composition functions, as summarized in Table 2.

According to the recommended experiment settings in [41], for each benchmark function in the CEC 2014 test suite, MPEPSO and the compared algorithms run 30 times independently, and the maximum number of function evaluations MaxFEs is set to $10,000 \mathrm{D}$. The experimental results 
TABle 1: Pseudocode of the MPEPSO algorithm.

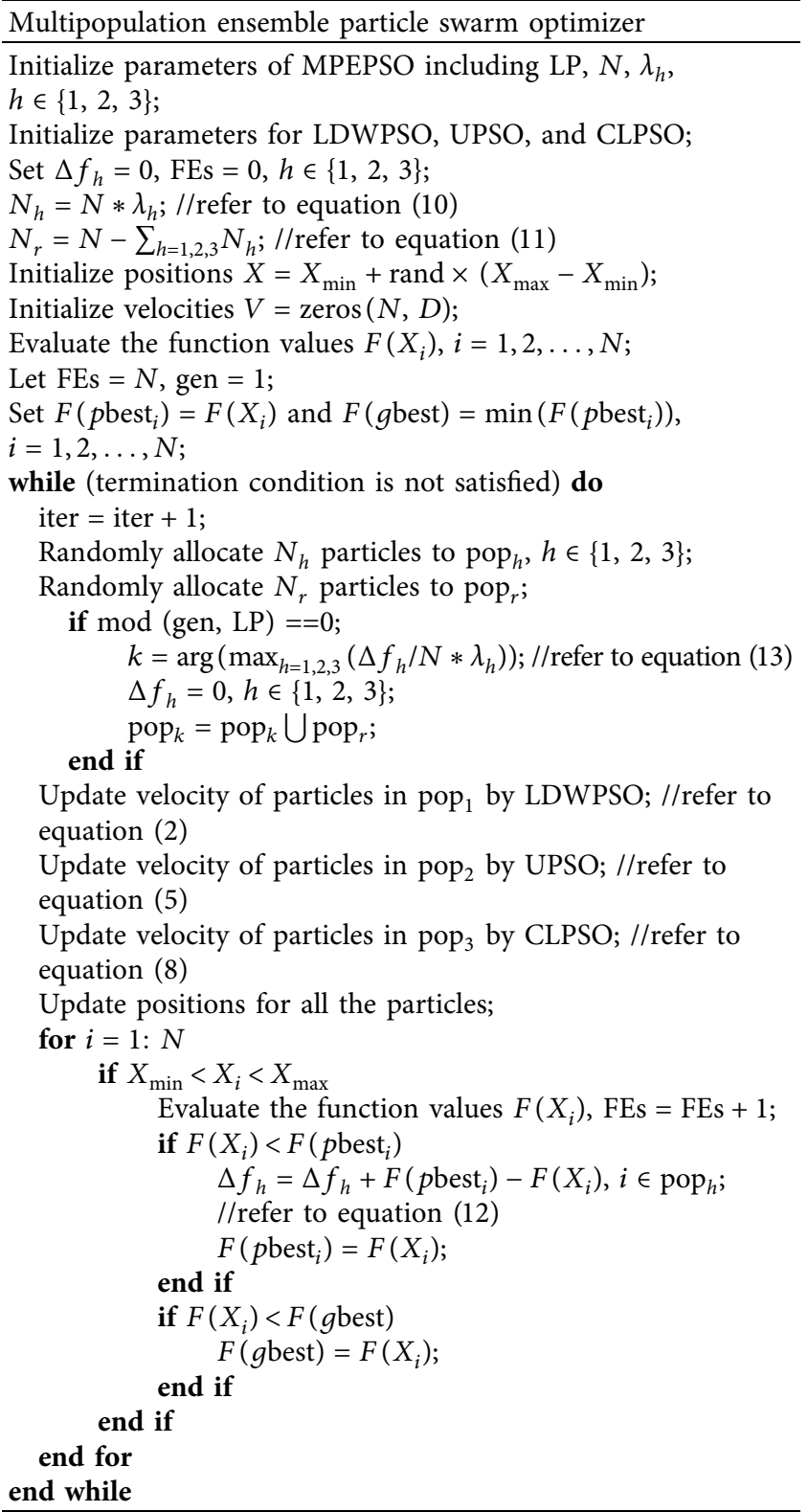

are obtained on both 10-dimensional and 30-dimensional problems.

The values of the mean error and standard deviation are recorded. Two evaluation measures are adopted in this paper. First, the algorithms are ranked based on the mean error values. For each benchmark function, the mean error values of the algorithms are ranked from the smallest to the highest. Second, the Wilcoxon signed-rank test is conducted at the $5 \%$ significance level to determine the significance of the difference between the mean error values obtained by MPEPSO and other algorithms. The null hypothesis is that the data in the vector $x$ and data in the vector $y$ come from a distribution with zero median. $h=1$ indicates a rejection of the null hypothesis, and $h=0$ indicates a failure to reject the null hypothesis at the 5\% significance level. Pairwise comparison is conducted over the results of 30 simulation runs between MPEPSO and other PSO variants. The symbol "+" represents that MPEPSO is significantly better than the compared algorithm, the symbol " $=$ " represents that there is no significant difference between MPEPSO and the compared algorithm, and the symbol "-" represents that MPEPSO is significantly worse than the compared algorithm.

MPEPSO is coded using MATLAB 2019b and executed on a computer with Intel Core i7-8565U CPU $(1.80 \mathrm{GHz})$, 8 GB RAM, and Windows 10 operating system.

4.2. Comparisons with PSO Variants. To comprehensively evaluate the performance of the proposed algorithm, the experimental results of MPEPSO are compared with six state-of-the-art PSO variants. These compared algorithms are listed as follows:

(i) LDWPSO: linearly decreasing inertia weight PSO [16]

(ii) UPSO: unified PSO [36]

(iii) CLPSO: comprehensive learning PSO [20]

(iv) DMS-PSO-HS: dynamic multiswarm particle swarm optimizer with harmony search [26]

(v) LISP: distance-based locally informed PSO [31]

(vi) SL-PSO: social learning PSO [43]

LDWPSO, UPSO, and CLPSO are classical PSO variants studied by many researchers, and they are the component strategy in the proposed algorithm. DMS-PSO-HS, LIPS, and SL-PSO are efficient PSO variants that attract many researchers' attention. Also, these three algorithms are developed by different mythologies. DMS-PSO-HS is developed by hybridizing PSO with other algorithms. LIPS is developed by proposing a new topology structure, distancebased neighborhood. SL-PSO introduces a new searching strategy. To comprehensively evaluate the performance of MPEPSO, these six PSO variants are used for the comparisons.

Shao et al. [26] proposed the DMS-PSO-HS algorithm by hybridizing the dynamic multiswarm PSO (DMS-PSO) [44] with the harmony search (HS) algorithm [45]. DMS-PSOHS has a better performance on most multimodal and unimodal problems comparing with the DMS-PSO and HS algorithm. LIPS [31], proposed by Qu et al., is designed to solve multimodal problems. In LIPS, particles are guided by several local bests. Inspired by social learning mechanisms, Cheng and Jin [43] proposed the SL-PSO algorithm. SL-PSO works consistently well on both low-dimensional problems and large-scale problems.

To conduct a fair competition, population size in SL-PSO is determined by the built-in function in the paper [43], and for algorithms that not mentioned how to set the population size $N$, the population size is set as $N=50$, which is a widely used configuration in population-based algorithms. The parameters for the comparison algorithms are set based on the original code of papers $[16,20,26,31,36,43]$.

The parameter settings for each algorithm are listed in Table 3. 
TABLE 2: CEC 2014 test suite (search range: $[-100,100]^{D}$ ).

\begin{tabular}{|c|c|c|c|}
\hline Type & No. & Function name & Minimum \\
\hline \multirow{3}{*}{ Unimodal functions } & F1 & Rotated High Conditioned Elliptic Function & 100 \\
\hline & $\mathrm{F} 2$ & Rotated Bent Cigar Function & 200 \\
\hline & F3 & Rotated Discus Function & 300 \\
\hline \multirow{13}{*}{ Simple multimodal functions } & F4 & Shifted and Rotated Rosenbrock's Function & 400 \\
\hline & F5 & Shifted and Rotated Ackley's Function & 500 \\
\hline & F6 & Shifted and Rotated Weierstrass Function & 600 \\
\hline & F7 & Shifted and Rotated Griewank's Function & 700 \\
\hline & F8 & Shifted Rastrigin's Function & 800 \\
\hline & F9 & Shifted and Rotated Rastrigin's Function & 900 \\
\hline & F10 & Shifted Schwefel's Function & 1000 \\
\hline & F11 & Shifted and Rotated Schwefel's Function & 1100 \\
\hline & F12 & Shifted and Rotated Katsuura Function & 1200 \\
\hline & F13 & Shifted and Rotated HappyCat Function & 1300 \\
\hline & F14 & Shifted and Rotated HGBat Function & 1400 \\
\hline & F15 & Shifted and Rotated Expanded Griewank's plus Rosenbrock's Function & 1500 \\
\hline & F16 & Shifted and Rotated Expanded Scaffer's F6 Function & 1600 \\
\hline \multirow{6}{*}{ Hybrid functions } & F17 & Hybrid Function $1(N=3)$ & 1700 \\
\hline & F18 & Hybrid Function $2(N=3)$ & 1800 \\
\hline & F19 & Hybrid Function $3(N=4)$ & 1900 \\
\hline & F20 & Hybrid Function $4(N=4)$ & 2000 \\
\hline & $\mathrm{F} 21$ & Hybrid Function $5(N=5)$ & 2100 \\
\hline & F22 & Hybrid Function $6(N=5)$ & 2200 \\
\hline \multirow{8}{*}{ Composition functions } & F23 & Composition Function $1(N=5)$ & 2300 \\
\hline & F24 & Composition Function $2(N=3)$ & 2400 \\
\hline & $\mathrm{F} 25$ & Composition Function $3(N=3)$ & 2500 \\
\hline & F26 & Composition Function $4(N=5)$ & 2600 \\
\hline & $\mathrm{F} 27$ & Composition Function $5(N=5)$ & 2700 \\
\hline & $\mathrm{F} 28$ & Composition Function $6(N=5)$ & 2800 \\
\hline & F29 & Composition Function $7(N=3)$ & 2900 \\
\hline & F30 & Composition Function $8(N=3)$ & 3000 \\
\hline
\end{tabular}

In DMS-PSO-HS, HMCR represents the harmony memory consideration rate, PAR is the pitch adjustment rate, SS is the number of subswarms, and SP is the subpopulation size of each subswarm. In LIPS, nsize is the neighborhood size. In SL-PSO, $M$ is the base swarm size, $\alpha$ and $\beta$ are two coefficients to tune the learning probability and the social influence, respectively.

According to the descriptions in CLPSO and LDWPSO $[16,20]$, the inertia weight $\omega$ is linearly decreased as shown in equation (3). In MPEPSO, the inertia weight $\omega$ is also linearly decreased from 0.9 to 0.4 . In addition, a time-varying acceleration coefficients approach [30] is also adopted, that is, the acceleration coefficients are linearly changed during the computation process. According to this study [30], $c_{1}$ is linearly decreasing from 2.5 to 0.5 and $c_{2}$ is increasing from 0.5 to 2.5 as shown in Table 3 . LP $=10$ and $\lambda_{h}=0.1$ are set empirically.

4.2.1. Computational Results on 10-Dimensional Problems. In this section, the performance of MPEPSO is compared with other PSO algorithms on 10 dimensions. The rank of each algorithm, mean error, and standard deviation values on 10-dimensional problems are shown in Table 4 . The results of Wilcoxon signed-rank tests are shown in Table 5. Furthermore, the $p$ value and the test decision $h$ of the Wilcoxon signed-rank tests are shown in Table 6.
For unimodal problems (F1 F3), MPEPSO ranks first for functions F1 and F3. For simple multimodal functions (F4 F16), MPEPSO gives the best results on functions F5, F14, and F16 and provides the second-best results on functions F6, F9, F11, F13, and F15. For hybrid functions (F17 F22), MPEPSO provides the best and second-best performance for all functions except for function F18, in which CLPSO outperforms the proposed algorithm according to the Wilcoxon signed-rank tests. For composition functions (F23 F30), MPEPSO performs within the top three for all the problems except for functions F28 and F30. Statistically, UPSO and CLPSO outperform the proposed algorithm for these two functions. Overall, MPEPSO has the best performance on 10 functions and the secondbest performance on 10 functions out of 30 functions.

As shown in Table 5, according to the results of Wilcoxon signed-rank tests, MPEPSO is significantly better than LDWPSO, UPSO, CLPSO, DMS-PSO-HS, LIPS, and SLPSO on 26, 19, 12, 21, 21, and 19 functions, respectively. It is inferior to LDWPSO, UPSO, CLPSO, DMS-PSO-HS, LIPS, and SL-PSO on $0,5,10,2,4$, and 4 functions while similar to them on $4,6,8,7,5$, and 7 functions, respectively.

The rank results over 30 problems are shown in Table 7. Algorithms are ranked based on the mean error values. MPEPSO has the best rank. The average rank of MPEPSO is 2.233333, while the rank of CLPSO is 2.866667. Also, the 
TABle 3: Parameter settings.

\begin{tabular}{lc}
\hline Algorithm & Parameter settings \\
\hline LDWPSO [16] & $\omega=0.9 \sim 0.4, c_{1}=c_{2}=2$ \\
UPSO [36] & $X=0.729, c_{1}=c_{2}=2.05, u=0.1, \mu=0, \sigma=0.01$ \\
CLPSO [20] & $\omega=0.9 \sim 0.2, c=1.49445, m=5, a=0, b=0.5$ \\
DMS-PSO-HS [26] & $\mathscr{X}=0.7298, n$ size $=3$ \\
LIPS [31] & $\omega=0.729, c_{1}=c_{2}=1.49445, \mathrm{HMCR}=0.98, \mathrm{PAR}=0.01 \sim 0.99, \mathrm{SS}=10, \mathrm{SP}=5$ \\
SL-PSO [43] & $N=M+D / 10, M=100, \beta=0.01, \alpha=1$ \\
MPEPSO & $\omega=0.9 \sim 0.4, c_{1}=2.5 \sim 0.5, c_{2}=0.5 \sim 2.5, \mathrm{LP}=10, \lambda_{h}=0.1$ \\
\hline
\end{tabular}

TABLE 4: Computational results on 10-dimensional problems.

\begin{tabular}{|c|c|c|c|c|c|c|c|c|}
\hline & & LDWPSO & UPSO & CLPSO & DMS-PSO-HS & LIPS & SL-PSO & MPEPSO \\
\hline \multirow{3}{*}{$\mathrm{F} 1$} & Rank & 2 & 3 & 5 & 6 & 7 & 4 & 1 \\
\hline & Mean & $2.08 E+04$ & $3.24 E+04$ & $9.52 E+04$ & $1.35 E+05$ & $1.73 E+05$ & $3.34 E+04$ & $1.04 E+03$ \\
\hline & Std & $2.04 E+04$ & $2.55 E+04$ & $7.43 E+04$ & $3.77 E+05$ & $2.01 E+05$ & $3.78 E+04$ & $9.65 E+02$ \\
\hline \multirow{3}{*}{$\mathrm{F} 2$} & Rank & 5 & 3 & 1 & 7 & 2 & 6 & 4 \\
\hline & Mean & $3.23 E+03$ & $8.98 E+02$ & $9.96 E+00$ & $2.44 E+05$ & $5.63 E+02$ & $3.27 E+03$ & $1.04 E+03$ \\
\hline & Std & $2.15 E+03$ & $1.62 E+03$ & $1.58 E+01$ & $1.29 E+06$ & $8.39 E+02$ & $3.43 E+03$ & $1.18 E+03$ \\
\hline \multirow{3}{*}{ F3 } & Rank & 6 & 5 & 2 & 3 & 4 & 7 & 1 \\
\hline & Mean & $7.73 E+02$ & $7.21 E+02$ & $1.05 E+02$ & $2.84 E+02$ & $3.30 E+02$ & $1.61 E+03$ & $2.53 E+01$ \\
\hline & Std & $1.06 E+03$ & $8.82 E+02$ & $1.16 E+02$ & $4.12 E+02$ & $3.07 E+02$ & $1.94 E+03$ & $5.06 E+01$ \\
\hline \multirow{3}{*}{$\mathrm{F} 4$} & Rank & 4 & 1 & 2 & 3 & 6 & 7 & 5 \\
\hline & Mean & $1.84 E+01$ & $5.19 E-01$ & $5.80 E-01$ & $1.60 E+01$ & $2.48 E+01$ & $3.48 E+01$ & $2.39 E+01$ \\
\hline & Std & $1.87 E+01$ & $3.31 E-01$ & $4.73 E-01$ & $1.21 E+01$ & $1.98 E+01$ & $2.67 E-14$ & $1.57 E+01$ \\
\hline \multirow{3}{*}{ F5 } & Rank & 7 & 6 & 3 & 4 & 5 & 2 & 1 \\
\hline & Mean & $2.03 E+01$ & $2.00 E+01$ & $1.92 E+01$ & $1.93 E+01$ & $1.94 E+01$ & $1.90 E+01$ & $1.62 E+01$ \\
\hline & Std & $7.45 E-02$ & $5.02 E-02$ & $2.77 E+00$ & $3.65 E+00$ & $3.56 E+00$ & $5.16 E+00$ & $8.23 E+00$ \\
\hline \multirow{3}{*}{ F6 } & Rank & 6 & 7 & 3 & 5 & 4 & 1 & 2 \\
\hline & Mean & $1.45 E+00$ & $2.22 E+00$ & $5.59 E-01$ & $9.96 E-01$ & $8.37 E-01$ & $0.00 E+00$ & $1.19 E-02$ \\
\hline & Std & $1.29 E+00$ & $1.06 E+00$ & $2.16 E-01$ & $8.02 E-01$ & $7.87 E-01$ & $0.00 E+00$ & $4.92 E-02$ \\
\hline \multirow{3}{*}{ F7 } & Rank & 6 & 3 & 5 & 7 & 1 & 2 & 4 \\
\hline & Mean & $1.35 E-01$ & $2.48 E-02$ & $3.12 E-02$ & $1.79 E-01$ & $5.01 E-03$ & $6.49 E-03$ & $3.08 E-02$ \\
\hline & Std & $7.70 E-02$ & $1.74 E-02$ & $1.41 E-02$ & $1.88 E-01$ & $5.07 E-03$ & $7.72 E-03$ & $2.14 E-02$ \\
\hline \multirow{3}{*}{ F8 } & Rank & 4 & 7 & 1 & 2 & 6 & 5 & 3 \\
\hline & Mean & $1.49 E+00$ & $6.86 E+00$ & $0.00 E+00$ & $5.66 E-01$ & $5.34 E+00$ & $4.21 E+00$ & $9.62 E-01$ \\
\hline & Std & $1.19 E+00$ & $3.40 E+00$ & $0.00 E+00$ & $1.22 E+00$ & $2.15 E+00$ & $2.00 E+00$ & $1.03 E+00$ \\
\hline \multirow{3}{*}{ F9 } & Rank & 5 & 7 & 3 & 6 & 4 & 1 & 2 \\
\hline & Mean & $7.76 E+00$ & $1.10 E+01$ & $5.05 E+00$ & $9.54 E+00$ & $6.50 E+00$ & $4.21 E+00$ & $4.78 E+00$ \\
\hline & Std & $2.77 E+00$ & $3.29 E+00$ & $1.26 E+00$ & $3.52 E+00$ & $2.49 E+00$ & $1.90 E+00$ & $1.89 E+00$ \\
\hline \multirow{3}{*}{ F10 } & Rank & 5 & 6 & 1 & 2 & 7 & 4 & 3 \\
\hline & Mean & $8.26 E+01$ & $1.81 E+02$ & $2.48 E-02$ & $2.89 E+01$ & $2.53 E+02$ & $4.82 E+01$ & $4.22 E+01$ \\
\hline & Std & $7.94 E+01$ & $1.09 E+02$ & $3.16 E-02$ & $5.03 E+01$ & $1.39 E+02$ & $4.04 E+01$ & $5.53 E+01$ \\
\hline \multirow{3}{*}{ F11 } & Rank & 4 & 7 & 3 & 6 & 5 & 1 & 2 \\
\hline & Mean & $2.63 E+02$ & $3.81 E+02$ & $1.52 E+02$ & $3.40 E+02$ & $3.14 E+02$ & $7.40 E+01$ & $1.08 E+02$ \\
\hline & Std & $1.66 E+02$ & $1.80 E+02$ & $7.14 E+01$ & $3.01 E+02$ & $1.20 E+02$ & $9.97 E+01$ & $1.04 E+02$ \\
\hline \multirow{3}{*}{ F12 } & Rank & 6 & 2 & 5 & 4 & 1 & 7 & 3 \\
\hline & Mean & $4.50 E-01$ & $1.12 E-01$ & $2.46 E-01$ & $2.31 E-01$ & $3.59 E-02$ & $1.03 E+00$ & $2.05 E-01$ \\
\hline & Std & $3.34 E-01$ & $5.31 E-02$ & $5.20 E-02$ & $1.86 E-01$ & $2.10 E-02$ & $4.26 E-01$ & $1.71 E-01$ \\
\hline \multirow{3}{*}{ F13 } & Rank & 6 & 5 & 7 & 4 & 3 & 1 & 2 \\
\hline & Mean & $1.49 E-01$ & $1.19 E-01$ & $1.55 E-01$ & $9.99 E-02$ & $7.67 E-02$ & $4.63 E-02$ & $7.19 E-02$ \\
\hline & Std & $4.80 E-02$ & $3.69 E-02$ & $3.63 E-02$ & $6.61 E-02$ & $3.52 E-02$ & $2.28 E-02$ & $3.33 E-02$ \\
\hline \multirow{3}{*}{ F14 } & Rank & 2 & 5 & 3 & 4 & 6 & 7 & 1 \\
\hline & Mean & $1.14 E-01$ & $1.89 E-01$ & $1.62 E-01$ & $1.65 E-01$ & $3.15 E-01$ & $3.95 E-01$ & $8.78 E-02$ \\
\hline & Std & $4.20 E-02$ & $4.63 E-02$ & $4.24 E-02$ & $6.02 E-02$ & $8.22 E-02$ & $8.40 E-02$ & $3.16 E-02$ \\
\hline \multirow{3}{*}{ F15 } & Rank & 6 & 3 & 5 & 7 & 1 & 4 & 2 \\
\hline & Mean & $1.01 E+00$ & $7.42 E-01$ & $9.45 E-01$ & $1.13 E+00$ & $5.86 E-01$ & $8.51 E-01$ & $6.79 E-01$ \\
\hline & Std & $4.14 E-01$ & $2.71 E-01$ & $1.62 E-01$ & $5.49 E-01$ & $1.42 E-01$ & $2.03 E-01$ & $1.60 E-01$ \\
\hline
\end{tabular}


TABle 4: Continued.

\begin{tabular}{|c|c|c|c|c|c|c|c|c|}
\hline & & LDWPSO & UPSO & CLPSO & DMS-PSO-HS & LIPS & SL-PSO & MPEPSO \\
\hline \multirow{3}{*}{ F16 } & Rank & 3 & 6 & 2 & 4 & 7 & 5 & 1 \\
\hline & Mean & $2.02 E+00$ & $2.48 E+00$ & $1.88 E+00$ & $2.20 E+00$ & $2.59 E+00$ & $2.45 E+00$ & $1.20 E+00$ \\
\hline & Std & $6.56 E-01$ & $3.59 E-01$ & $3.46 E-01$ & $5.19 E-01$ & $3.75 E-01$ & $3.68 E-01$ & $5.78 E-01$ \\
\hline \multirow{3}{*}{ F17 } & Rank & 3 & 2 & 5 & 4 & 7 & 6 & 1 \\
\hline & Mean & $3.82 E+03$ & $2.72 E+03$ & $4.29 E+03$ & $4.16 E+03$ & $1.81 E+04$ & $9.57 E+03$ & $8.92 E+02$ \\
\hline & Std & $3.00 E+03$ & $1.83 E+03$ & $3.52 E+03$ & $1.35 E+04$ & $2.39 E+04$ & $1.18 E+04$ & $9.33 E+02$ \\
\hline \multirow{3}{*}{ F18 } & Rank & 7 & 2 & 1 & 5 & 4 & 6 & 3 \\
\hline & Mean & $1.21 E+04$ & $4.47 E+02$ & $2.45 E+01$ & $3.51 E+03$ & $1.65 E+03$ & $7.93 E+03$ & $9.82 E+02$ \\
\hline & Std & $9.91 E+03$ & $4.52 E+02$ & $1.94 E+01$ & $3.71 E+03$ & $2.48 E+03$ & $4.20 E+03$ & $1.06 E+03$ \\
\hline \multirow{3}{*}{ F19 } & Rank & 5 & 6 & 2 & 4 & 7 & 3 & 1 \\
\hline & Mean & $1.42 E+00$ & $1.43 E+00$ & $3.69 E-01$ & $1.02 E+00$ & $2.03 E+00$ & $1.02 E+00$ & $2.77 E-01$ \\
\hline & Std & $6.36 E-01$ & $3.37 E-01$ & $1.69 E-01$ & $7.16 E-01$ & $5.77 E-01$ & $5.82 E-01$ & $3.83 E-01$ \\
\hline \multirow{3}{*}{ F20 } & Rank & 3 & 4 & 1 & 6 & 5 & 7 & 2 \\
\hline & Mean & $3.03 E+02$ & $3.33 E+02$ & $1.65 E+01$ & $9.34 E+02$ & $6.47 E+02$ & $3.58 E+03$ & $5.27 E+01$ \\
\hline & Std & $8.33 E+02$ & $3.18 E+02$ & $3.01 E+01$ & $1.63 E+03$ & $8.35 E+02$ & $2.46 E+03$ & $6.38 E+01$ \\
\hline \multirow{3}{*}{$\mathrm{F} 21$} & Rank & 2 & 3 & 4 & 5 & 6 & 7 & 1 \\
\hline & Mean & $1.12 E+02$ & $6.80 E+02$ & $6.85 E+02$ & $7.40 E+02$ & $1.01 E+03$ & $6.43 E+03$ & $3.30 E+01$ \\
\hline & Std & $1.02 E+02$ & $8.30 E+02$ & $5.69 E+02$ & $1.01 E+03$ & $1.38 E+03$ & $5.59 E+03$ & $4.70 E+01$ \\
\hline \multirow{3}{*}{ F22 } & Rank & 5 & 6 & 1 & 3 & 7 & 4 & 2 \\
\hline & Mean & $1.25 E+01$ & $2.21 E+01$ & $1.01 E+00$ & $7.85 E+00$ & $5.20 E+01$ & $1.03 E+01$ & $7.32 E+00$ \\
\hline & Std & $1.00 E+01$ & $2.03 E+00$ & $1.87 E+00$ & $1.03 E+01$ & $4.44 E+01$ & $1.21 E+01$ & $9.52 E+00$ \\
\hline \multirow{3}{*}{ F23 } & Rank & 2 & 2 & 1 & 7 & 2 & 2 & 2 \\
\hline & Mean & $3.29 E+02$ & $3.29 E+02$ & $2.94 E+02$ & $3.29 E+02$ & $3.29 E+02$ & $3.29 E+02$ & $3.29 E+02$ \\
\hline & Std & $2.38 E-13$ & $2.89 E-13$ & $9.13 E+01$ & $7.83 E-02$ & $5.25 E-13$ & $2.27 E-13$ & $2.89 E-13$ \\
\hline \multirow{3}{*}{ F24 } & Rank & 4 & 7 & 3 & 5 & 6 & 1 & 2 \\
\hline & Mean & $1.19 E+02$ & $1.20 E+02$ & $1.13 E+02$ & $1.19 E+02$ & $1.19 E+02$ & $1.10 E+02$ & $1.11 E+02$ \\
\hline & Std & $5.29 E+00$ & $3.94 E+00$ & $1.82 E+00$ & $7.14 E+00$ & $7.96 E+00$ & $2.83 E+00$ & $2.43 E+00$ \\
\hline \multirow{3}{*}{ F25 } & Rank & 7 & 5 & 2 & 3 & 4 & 6 & 1 \\
\hline & Mean & $1.87 E+02$ & $1.73 E+02$ & $1.39 E+02$ & $1.56 E+02$ & $1.71 E+02$ & $1.81 E+02$ & $1.35 E+02$ \\
\hline & Std & $3.16 E+01$ & $2.44 E+01$ & $1.08 E+01$ & $2.69 E+01$ & $2.84 E+01$ & $3.39 E+01$ & $2.79 E+01$ \\
\hline \multirow{3}{*}{ F26 } & Rank & 6 & 4 & 7 & 5 & 3 & 1 & 2 \\
\hline & Mean & $1.00 E+02$ & $1.00 E+02$ & $1.00 E+02$ & $1.00 E+02$ & $1.00 E+02$ & $1.00 E+02$ & $1.00 E+02$ \\
\hline & Std & $4.82 E-02$ & $3.45 E-02$ & $3.15 E-02$ & $6.05 E-02$ & $4.66 E-02$ & $1.66 E-02$ & $2.18 E-02$ \\
\hline \multirow{3}{*}{ F27 } & Rank & 5 & 7 & 2 & 3 & 6 & 4 & 1 \\
\hline & Mean & $2.37 E+02$ & $3.22 E+02$ & $8.31 E+01$ & $1.44 E+02$ & $2.88 E+02$ & $2.07 E+02$ & $5.16 E+01$ \\
\hline & Std & $1.72 E+02$ & $1.61 E+02$ & $1.38 E+02$ & $1.86 E+02$ & $9.90 E+01$ & $1.48 E+02$ & $1.31 E+02$ \\
\hline \multirow{3}{*}{ F28 } & Rank & 7 & 1 & 2 & 6 & 3 & 4 & 5 \\
\hline & Mean & $4.81 E+02$ & $3.06 E+02$ & $3.65 E+02$ & $4.37 E+02$ & $3.95 E+02$ & $4.06 E+02$ & $4.22 E+02$ \\
\hline & Std & $6.33 E+01$ & $6.02 E-02$ & $8.25 E+00$ & $1.13 E+02$ & $1.24 E+02$ & $4.10 E+01$ & $5.35 E+01$ \\
\hline \multirow{3}{*}{ F29 } & Rank & 7 & 1 & 2 & 4 & 6 & 5 & 3 \\
\hline & Mean & $2.00 E+05$ & $2.04 E+02$ & $2.69 E+02$ & $3.31 E+02$ & $8.21 E+02$ & $4.96 E+02$ & $2.87 E+02$ \\
\hline & Std & $7.61 E+05$ & $1.09 E+00$ & $2.50 E+01$ & $1.17 E+02$ & $1.42 E+03$ & $1.72 E+02$ & $3.62 E+01$ \\
\hline \multirow{3}{*}{ F30 } & Rank & 5 & 1 & 2 & 6 & 7 & 3 & 4 \\
\hline & Mean & $8.58 E+02$ & $2.62 E+02$ & $5.88 E+02$ & $9.48 E+02$ & $1.20 E+03$ & $6.51 E+02$ & $7.33 E+02$ \\
\hline & Std & $3.46 E+02$ & $3.34 E+01$ & $6.05 E+01$ & $2.44 E+02$ & $3.15 E+02$ & $2.00 E+02$ & $1.86 E+02$ \\
\hline
\end{tabular}

average ranks of other algorithms are larger than 4 . Therefore, MPEPSO performs well for the CEC 2014 test suite on 10-dimensional problems in comparison with the other six PSO variants.

In addition, the best values of MPEPSO and other algorithms are shown in Table 8. MPEPSO obtains better values on 10 problems. The worst values of these algorithms are shown in Table 9. MPEPSO obtains the best results in 8 problems.

The median convergence graphs of six functions are shown in Figure 2. These functions are selected from four types of functions in the CEC 2014 test suite. For unimodal problems, the convergence graphs of $\mathrm{F} 1$ and $\mathrm{F} 3$ are shown in Figures 2(a) and 2(b). As shown in the figure, MPEPSO performs well for both functions. Also, the convergence speed of MPEPSO on F1 is much faster than other algorithms. However, the result obtained by MPEPSO is close to the result obtained by CLPSO on F3. For simple multimodal functions, the convergence graph of F16 is shown in Figure 2(c). It can be observed that MPEPSO has a faster convergence speed than other algorithms. For hybrid problems, F17 and F21 are shown in Figures 2(d) and 2(e). 
TABLE 5: Wilcoxon signed-rank test for 30 runs on 10-dimensional problems.

\begin{tabular}{|c|c|c|c|c|c|c|}
\hline & LDWPSO & UPSO & CLPSO & DMS-PSO-HS & LIPS & SL-PSO \\
\hline F1 & + & + & + & + & + & + \\
\hline F2 & + & $=$ & - & $=$ & - & + \\
\hline F3 & + & + & + & + & + & + \\
\hline $\mathrm{F} 4$ & $=$ & - & - & - & $=$ & + \\
\hline F5 & + & $=$ & $=$ & $=$ & $=$ & + \\
\hline F6 & + & + & + & + & + & - \\
\hline F7 & + & $=$ & $=$ & + & - & - \\
\hline F8 & + & + & - & $=$ & + & + \\
\hline F9 & + & + & $=$ & + & + & $=$ \\
\hline F10 & + & + & - & - & + & $=$ \\
\hline F11 & + & + & $=$ & + & + & $=$ \\
\hline F12 & + & - & $=$ & $=$ & - & + \\
\hline F13 & + & + & + & + & $=$ & - \\
\hline F14 & + & + & + & + & + & + \\
\hline F15 & + & $=$ & + & + & - & + \\
\hline F16 & + & + & + & + & + & + \\
\hline F17 & + & + & + & + & + & + \\
\hline F18 & + & $=$ & - & + & $=$ & + \\
\hline F19 & + & + & $=$ & + & + & + \\
\hline F20 & $=$ & + & - & + & + & + \\
\hline F21 & + & + & + & + & + & + \\
\hline F22 & $=$ & + & - & $=$ & + & $=$ \\
\hline $\mathrm{F} 23$ & + & $=$ & $=$ & + & + & + \\
\hline F24 & + & + & + & + & + & $=$ \\
\hline F25 & + & + & $=$ & + & + & + \\
\hline F26 & + & + & + & + & + & - \\
\hline F27 & + & + & + & + & + & + \\
\hline F28 & + & - & - & $=$ & $=$ & $=$ \\
\hline F29 & + & - & - & $=$ & + & + \\
\hline F30 & $=$ & - & - & + & + & $=$ \\
\hline$+/=/-$ & $26 / 4 / 0$ & $\begin{array}{c}19 / 6 / \\
5\end{array}$ & $\begin{array}{c}12 / 8 / \\
10\end{array}$ & $21 / 7 / 2$ & $\begin{array}{l}21 / \\
5 / 4\end{array}$ & $19 / 7 / 4$ \\
\hline
\end{tabular}

For both functions, MPEPSO obtains the best results and converges fast at the early stage on F17, while DMS-PSO-HS also performs well on F17. For composition functions, the convergence graphs of F24 are selected. MPEPSO has a very fast convergence speed and obtains the best result.

4.2.2. Computational Results on 30-Dimensional Problems. In this section, the performance of MPEPSO is compared with other PSO algorithms on 30 dimensions. The computational results are shown in Table 10, and the results of Wilcoxon signed-rank tests are shown in Table 11. Also, the $p$ value and the test decision $h$ of the Wilcoxon signed-rank tests are shown in Table 12.

For unimodal functions $(\mathrm{F} 1 \sim \mathrm{F} 3)$, MPEPSO provides the best solutions for all three functions. For simple multimodal functions (F4 F16), MPEPSO ranks first for functions F4 and F14 F16 and ranks second for functions F6, F9, and F13. For hybrid functions (F17 F22), MPEPSO gives the best solutions for all functions except for function F18. Statistically, CLPSO outperforms MPEPSO on this function. For composition functions (F23 F30), MPEPSO performs within the top three for all the functions. MPEPSO has the best performance on 12 functions and the secondbest performance on 7 functions out of 30 functions.

As shown in Table 11, according to the results of Wilcoxon signed-rank tests, MPEPSO is significantly better than LDWPSO, UPSO, CLPSO, DMS-PSO-HS, LIPS, and SLPSO on $26,20,17,28,24$, and 16 functions, respectively. It is inferior to LDWPSO, UPSO, CLPSO, DMS-PSO-HS, LIPS, and SL-PSO on $0,7,9,1,3$, and 7 functions while similar to them on 4, 3, 4, 1, 3 and 7 functions, respectively.

As shown in Table 13, the average rank of MPEPSO is 2.2. The average ranks of other algorithms are much larger than the average rank of MPEPSO. MPEPSO ranks first for the CEC 2014 test suite on 30-dimensional problems in comparison with the other six PSO variants.

Furthermore, Table 14 shows the best values of seven algorithms on 30-dimensional problems. MPEPSO ranks first on 12 problems. The worst values of these algorithms are shown in Table 15. MPEPSO obtains the best results on 10 problems.

The median convergence graphs of six functions are shown in Figure 3. The same functions are selected as in Section 4.2. Overall, the convergence behaviors of MPEPSO on 30 dimensions and 10 dimensions are similar. However, for hybrid function F21, MPEPSO performs better on 30 dimensions than 10 dimensions. All the algorithms have similar performance on F25, and MPESPO performs worse than UPSO, SL-PSO, and CLPSO.

4.3. Comparisons with Other Metaheuristic Algorithms. To further investigate the effectiveness of MPEPSO, the performance of MPEPSO is compared with that of other metaheuristic algorithms. These compared algorithms are listed as follows:

(i) CS: cuckoo search [46]

(ii) FA: firefly algorithm [47]

(iii) SCA: sine cosine algorithm [48]

(iv) GCMBO: monarch butterfly optimization with greedy strategy and crossover operator [49]

(v) L-SHADE: success-history-based adaptive DE with linear population size reduction [50]

CS [46] and FA [47] are classical nature-inspired metaheuristic algorithms that have received considerable scholarly attention. L-SHADE [50] is the winner of the CEC 2014 competition. These algorithms also have been successfully applied to solve real-world problems, such as underwater glider path planning [51]. A recent study indicates that FA is the most successful among nature-inspired algorithms on the CEC 2014 test suite, while CS has better performance compared with FA on 10-dimensional problems [52]. This study also shows that L-SHADE is still one of the most effective algorithms on the CEC 2014 test suite [52]. We also compare our algorithm with two recently proposed algorithms, SCA [48] and GCMBO [49].

The parameter settings for each algorithm are listed in Table 16. The parameters for CS and FA are set based on the 
TABLE 6: The $p$ value and $h$ of the Wilcoxon signed-rank test on 10 -dimensional problems.

\begin{tabular}{|c|c|c|c|c|c|c|c|c|c|c|c|c|}
\hline & \multicolumn{2}{|c|}{ LDWPSO } & \multicolumn{2}{|c|}{ UPSO } & \multicolumn{2}{|c|}{ CLPSO } & \multicolumn{2}{|c|}{ DMS-PSO-HS } & \multicolumn{2}{|l|}{ LIPS } & \multicolumn{2}{|c|}{ SL-PSO } \\
\hline & $p$ value & $h$ & $p$ value & $h$ & $p$ value & $h$ & $p$ value & $h$ & $p$ value & $h$ & $p$ value & $h$ \\
\hline $\mathrm{F} 1$ & $5.75 E-06$ & 1 & $2.35 E-06$ & 1 & $1.73 E-06$ & 1 & $1.73 E-06$ & 1 & $1.73 E-06$ & 1 & $2.88 E-06$ & 1 \\
\hline F2 & $2.84 E-05$ & 1 & $1.31 E-01$ & 0 & $2.13 E-06$ & 1 & $1.71 E-01$ & 0 & $3.87 E-02$ & 1 & $7.73 E-03$ & 1 \\
\hline F3 & $1.73 E-06$ & 1 & $4.73 E-06$ & 1 & $3.72 E-05$ & 1 & $7.51 E-05$ & 1 & $1.73 E-06$ & 1 & $3.52 E-06$ & 1 \\
\hline F4 & $7.66 E-01$ & 0 & $6.34 E-06$ & 1 & $7.69 E-06$ & 1 & $3.00 E-02$ & 1 & $3.71 E-01$ & 0 & $1.75 E-02$ & 1 \\
\hline F5 & $3.00 E-02$ & 1 & $2.99 E-01$ & 0 & $2.89 E-01$ & 0 & $1.65 E-01$ & 0 & $1.65 E-01$ & 0 & $1.04 E-02$ & 1 \\
\hline F6 & $2.13 E-06$ & 1 & $1.73 E-06$ & 1 & $1.73 E-06$ & 1 & $1.73 E-06$ & 1 & $1.92 E-06$ & 1 & $1.23 E-05$ & 1 \\
\hline F7 & $1.73 E-06$ & 1 & $2.21 E-01$ & 0 & $6.88 E-01$ & 0 & $5.22 E-06$ & 1 & $3.51 E-06$ & 1 & $7.69 E-06$ & 1 \\
\hline F8 & $4.83 E-02$ & 1 & $1.73 E-06$ & 1 & $1.36 E-04$ & 1 & $1.71 E-01$ & 0 & $1.69 E-06$ & 1 & $3.53 E-06$ & 1 \\
\hline F9 & $2.08 E-04$ & 1 & $2.88 E-06$ & 1 & $3.39 E-01$ & 0 & $3.52 E-06$ & 1 & $1.07 E-02$ & 1 & $2.34 E-01$ & 0 \\
\hline F10 & $5.83 E-03$ & 1 & $2.60 E-06$ & 1 & $1.73 E-06$ & 1 & $3.00 E-02$ & 1 & $4.29 E-06$ & 1 & $3.29 E-01$ & 0 \\
\hline F11 & $2.05 E-04$ & 1 & $1.02 E-05$ & 1 & $7.19 E-02$ & 0 & $7.71 E-04$ & 1 & $6.98 E-06$ & 1 & $1.20 E-01$ & 0 \\
\hline F12 & $4.20 E-04$ & 1 & $2.30 E-02$ & 1 & $1.53 E-01$ & 0 & $2.89 E-01$ & 0 & $1.80 E-05$ & 1 & $3.88 E-06$ & 1 \\
\hline F13 & $3.52 E-06$ & 1 & $4.07 E-05$ & 1 & $1.92 E-06$ & 1 & $2.70 E-02$ & 1 & $4.41 E-01$ & 0 & $2.58 E-03$ & 1 \\
\hline F14 & $2.56 E-02$ & 1 & $2.35 E-06$ & 1 & $6.34 E-06$ & 1 & $1.24 E-05$ & 1 & $1.73 E-06$ & 1 & $1.73 E-06$ & 1 \\
\hline F15 & $4.53 E-04$ & 1 & $3.39 E-01$ & 0 & $1.97 E-05$ & 1 & $1.48 E-04$ & 1 & $3.16 E-02$ & 1 & $7.71 E-04$ & 1 \\
\hline F16 & $3.32 E-04$ & 1 & $1.73 E-06$ & 1 & $7.51 E-05$ & 1 & $8.47 E-06$ & 1 & $1.92 E-06$ & 1 & $1.73 E-06$ & 1 \\
\hline F17 & $2.16 E-05$ & 1 & $6.32 E-05$ & 1 & $9.32 E-06$ & 1 & $3.87 E-02$ & 1 & $2.35 E-06$ & 1 & $3.18 E-06$ & 1 \\
\hline F18 & $9.32 E-06$ & 1 & $5.71 E-02$ & 0 & $3.18 E-06$ & 1 & $6.84 E-03$ & 1 & $1.85 E-01$ & 0 & $3.52 E-06$ & 1 \\
\hline F19 & $1.73 E-06$ & 1 & $2.13 E-06$ & 1 & $1.53 E-01$ & 0 & $1.06 E-04$ & 1 & $1.73 E-06$ & 1 & $8.47 E-06$ & 1 \\
\hline F20 & $2.45 E-01$ & 0 & $6.34 E-06$ & 1 & $8.31 E-04$ & 1 & $1.89 E-04$ & 1 & $6.98 E-06$ & 1 & $1.73 E-06$ & 1 \\
\hline $\mathrm{F} 21$ & $7.16 E-04$ & 1 & $1.73 E-06$ & 1 & $1.73 E-06$ & 1 & $6.98 E-06$ & 1 & $2.60 E-06$ & 1 & $1.73 E-06$ & 1 \\
\hline F22 & $2.13 E-01$ & 0 & $6.98 E-06$ & 1 & $1.48 E-02$ & 1 & $8.77 E-01$ & 0 & $1.73 E-06$ & 1 & $4.17 E-01$ & 0 \\
\hline F23 & $9.63 E-07$ & 1 & 1 & 0 & $5.71 E-02$ & 0 & $1.73 E-06$ & 1 & $4.33 E-06$ & 1 & $3.91 E-03$ & 1 \\
\hline F24 & $6.98 E-06$ & 1 & $1.92 E-06$ & 1 & $4.45 E-05$ & 1 & $2.60 E-05$ & 1 & $5.75 E-06$ & 1 & $5.04 E-01$ & 0 \\
\hline F25 & $2.83 E-04$ & 1 & $2.84 E-05$ & 1 & $5.98 E-02$ & 0 & $2.96 E-03$ & 1 & $2.61 E-04$ & 1 & $2.05 E-04$ & 1 \\
\hline F26 & $9.32 E-06$ & 1 & $2.35 E-06$ & 1 & $1.73 E-06$ & 1 & $8.19 E-05$ & 1 & $2.60 E-05$ & 1 & $3.85 E-03$ & 1 \\
\hline F27 & $1.48 E-04$ & 1 & $4.29 E-06$ & 1 & $2.77 E-03$ & 1 & $1.60 E-04$ & 1 & $1.02 E-05$ & 1 & $8.94 E-04$ & 1 \\
\hline F28 & $6.16 E-04$ & 1 & $1.73 E-06$ & 1 & $5.79 E-05$ & 1 & $2.37 E-01$ & 0 & $6.73 E-01$ & 0 & $3.39 E-01$ & 0 \\
\hline F29 & $1.73 E-06$ & 1 & $1.73 E-06$ & 1 & $4.49 E-02$ & 1 & $4.65 E-01$ & 0 & $2.60 E-06$ & 1 & $3.88 E-06$ & 1 \\
\hline F30 & $3.18 E-01$ & 0 & $1.73 E-06$ & 1 & $1.74 E-04$ & 1 & $3.59 E-04$ & 1 & $4.73 E-06$ & 1 & $1.06 E-01$ & 0 \\
\hline
\end{tabular}

TABLe 7: The rank of the seven algorithms on 10-dimensional problems.

\begin{tabular}{|c|c|c|c|c|c|c|c|}
\hline Rank & LDWPSO & UPSO & CLPSO & DMS-PSO-HS & LIPS & SL-PSO & MPEPSO \\
\hline Total rank & 145 & 127 & 86 & 140 & 142 & 123 & 67 \\
\hline Ave. rank & 4.833333 & 4.233333 & 2.866667 & 4.666667 & 4.733333 & 4.1 & 2.233333 \\
\hline Final rank & 7 & 4 & 2 & 5 & 6 & 3 & 1 \\
\hline
\end{tabular}

study on CEC 2014 [52]. The parameters for SCA, GCMBO, and L-SHADE are set based on the original paper [48-50].

4.3.1. Computational Results on 10-Dimensional Problems. In this section, the performance of MPEPSO is compared with that of other metaheuristic algorithms on 10 dimensions. The rank of each algorithm, mean error, and standard deviation values on 10-dimensional problems are shown in Table 17. The results of Wilcoxon signed-rank tests are shown in Table 18.

For unimodal functions (F1 F3), CS and L-SHADE provide better solutions comparing with MPEPSO, while MPEPSO performances better than other algorithms. For simple multimodal functions (F4 F16), MPEPSO ranks first for function F16 and ranks second for functions F4 F7, F9, F11, F14, and F15. For hybrid functions (F17 F22), MPEPSO gives the second-best solutions on function F19 and F22, while L-SHADE provides the best solutions on all the functions. For composition functions
(F23 F30), MPEPSO performs best on function F25. MPEPSO has the best performance on 2 functions and the second-best performance on 14 functions out of 30 functions.

As shown in Table 18, according to the results of Wilcoxon signed-rank tests, MPEPSO is significantly better than CS, FA, SCA, GCMBO, and L-SHADE on 13, 19, 30, 29, and 0 functions, respectively. It is inferior to CS, FA, SCA, GCMBO, and L-SHADE on 12, 2, 0, 0, and 22 functions while similar to them on $5,9,0,1$, and 8 functions, respectively.

As shown in Table 19, the average rank of MPEPSO is 2.5. L-SHADE ranks first for the CEC 2014 test suite on 10dimensional problems.

4.3.2. Computational Results on 30-Dimensional Problems. In this section, the performance of MPEPSO is compared with that of other metaheuristic algorithms on 30 
TABLE 8: Best values of function error values for 30 runs on 10-dimensional problems.

\begin{tabular}{|c|c|c|c|c|c|c|c|}
\hline & LDWPSO & UPSO & CLPSO & DMS-PSO-HS & LIPS & SL-PSO & MPEPSO \\
\hline $\mathrm{F} 1$ & $5.02 E+02$ & $3.30 E+02$ & $1.36 E+04$ & $2.66 E+03$ & $4.77 E+03$ & $1.04 E+03$ & $1.05 E+01$ \\
\hline $\mathrm{F} 2$ & $1.93 E+02$ & $5.20 E-01$ & $6.01 E-02$ & $2.52 E-01$ & $1.93 E-01$ & $4.54 E+00$ & $2.31 E+00$ \\
\hline F3 & $4.76 E+01$ & $1.10 E+01$ & $2.61 E+00$ & $1.70 E+00$ & $1.40 E+01$ & $5.08 E+00$ & $2.90 E-02$ \\
\hline $\mathrm{F} 4$ & $1.53 E-02$ & $3.32 E-04$ & $7.64 E-02$ & $2.71 E-01$ & $2.52 E-06$ & $3.48 E+01$ & $2.30 E-02$ \\
\hline F5 & $2.01 E+01$ & $2.00 E+01$ & $8.63 E+00$ & $3.06 E-04$ & $5.21 E-01$ & $0.00 E+00$ & $0.00 E+00$ \\
\hline F6 & $0.00 E+00$ & $4.58 E-02$ & $1.90 E-01$ & $1.36 E-02$ & $1.60 E-05$ & $0.00 E+00$ & $0.00 E+00$ \\
\hline F7 & $3.70 E-02$ & $1.14 E-13$ & $4.22 E-03$ & $1.23 E-02$ & $0.00 E+00$ & $0.00 E+00$ & $7.40 E-03$ \\
\hline F8 & $0.00 E+00$ & $1.99 E+00$ & $0.00 E+00$ & $0.00 E+00$ & $9.95 E-01$ & $9.95 E-01$ & $0.00 E+00$ \\
\hline F9 & $3.98 E+00$ & $4.97 E+00$ & $2.15 E+00$ & $3.98 E+00$ & $2.98 E+00$ & $0.00 E+00$ & $9.95 E-01$ \\
\hline F10 & $3.48 E+00$ & $6.89 E+00$ & $2.71 E-06$ & $6.25 E-02$ & $3.48 E+00$ & $1.87 E-01$ & $1.87 E-01$ \\
\hline F11 & $6.83 E+00$ & $1.69 E+01$ & $6.27 E+01$ & $1.29 E+01$ & $4.68 E+01$ & $1.87 E-01$ & $3.66 E+00$ \\
\hline F12 & $5.35 E-02$ & $2.01 E-02$ & $1.34 E-01$ & $4.60 E-02$ & $1.99 E-03$ & $2.71 E-02$ & $1.38 E-02$ \\
\hline F13 & $6.82 E-02$ & $6.75 E-02$ & $8.10 E-02$ & $2.16 E-02$ & $2.12 E-02$ & $1.36 E-02$ & $1.48 E-02$ \\
\hline F14 & $2.84 E-02$ & $1.07 E-01$ & $8.62 E-02$ & $5.50 E-02$ & $1.44 E-01$ & $1.82 E-01$ & $3.27 E-02$ \\
\hline F15 & $4.22 E-01$ & $2.86 E-01$ & $6.24 E-01$ & $2.49 E-01$ & $3.00 E-01$ & $4.76 E-01$ & $3.78 E-01$ \\
\hline F16 & $3.87 E-01$ & $1.38 E+00$ & $8.50 E-01$ & $1.37 E+00$ & $1.83 E+00$ & $1.66 E+00$ & $2.34 E-01$ \\
\hline F17 & $1.81 E+02$ & $3.62 E+02$ & $3.93 E+02$ & $1.52 E+02$ & $1.38 E+03$ & $8.89 E+02$ & $2.67 E+01$ \\
\hline F18 & $1.42 E+02$ & $3.62 E+01$ & $5.08 E+00$ & $5.42 E+00$ & $3.92 E+01$ & $1.97 E+02$ & $1.22 E+01$ \\
\hline F19 & $1.84 E-01$ & $2.12 E-01$ & $8.04 E-02$ & $8.44 E-02$ & $1.00 E+00$ & $1.69 E-01$ & $1.94 E-02$ \\
\hline F20 & $3.93 E+00$ & $2.37 E+01$ & $2.97 E+00$ & $4.61 E+00$ & $3.50 E+01$ & $7.29 E+02$ & $3.31 E+00$ \\
\hline F21 & $3.09 E+00$ & $1.27 E+01$ & $6.59 E+01$ & $5.16 E-01$ & $4.08 E+01$ & $1.45 E+00$ & $3.04 E-01$ \\
\hline F22 & $4.47 E-04$ & $1.85 E+01$ & $2.78 E-01$ & $7.18 E-02$ & $1.98 E+01$ & $1.27 E-03$ & $2.09 E-02$ \\
\hline F23 & $3.29 E+02$ & $3.29 E+02$ & $2.63 E+01$ & $3.29 E+02$ & $3.29 E+02$ & $3.29 E+02$ & $3.29 E+02$ \\
\hline F24 & $1.09 E+02$ & $1.11 E+02$ & $1.10 E+02$ & $1.08 E+02$ & $1.09 E+02$ & $1.05 E+02$ & $1.06 E+02$ \\
\hline F25 & $1.13 E+02$ & $1.29 E+02$ & $1.20 E+02$ & $1.14 E+02$ & $1.24 E+02$ & $1.16 E+02$ & $1.00 E+02$ \\
\hline F26 & $1.00 E+02$ & $1.00 E+02$ & $1.00 E+02$ & $1.00 E+02$ & $1.00 E+02$ & $1.00 E+02$ & $1.00 E+02$ \\
\hline F27 & $1.70 E+00$ & $3.67 E+00$ & $3.37 E+00$ & $2.12 E+00$ & $5.58 E+00$ & $9.23 E-01$ & $9.59 E-01$ \\
\hline F28 & $3.72 E+02$ & $3.06 E+02$ & $3.57 E+02$ & $1.02 E+02$ & $1.00 E+02$ & $3.69 E+02$ & $3.57 E+02$ \\
\hline F29 & $2.93 E+02$ & $2.03 E+02$ & $2.28 E+02$ & $2.19 E+02$ & $2.82 E+02$ & $2.68 E+02$ & $2.34 E+02$ \\
\hline F30 & $5.43 E+02$ & $2.26 E+02$ & $5.00 E+02$ & $5.57 E+02$ & $6.55 E+02$ & $4.81 E+02$ & $5.23 E+02$ \\
\hline
\end{tabular}

TABLE 9: Worst values of function error values for 30 runs on 10-dimensional problems.

\begin{tabular}{|c|c|c|c|c|c|c|c|}
\hline & LDWPSO & UPSO & CLPSO & DMS-PSO-HS & LIPS & SL-PSO & MPEPSO \\
\hline $\mathrm{F} 1$ & $8.32 E+04$ & $1.05 E+05$ & $4.11 E+05$ & $2.07 E+06$ & $6.29 E+05$ & $1.31 E+05$ & $4.40 E+03$ \\
\hline F2 & $9.66 E+03$ & $7.76 E+03$ & $7.45 E+01$ & $7.09 E+06$ & $3.91 E+03$ & $1.14 E+04$ & $4.22 E+03$ \\
\hline F3 & $5.54 E+03$ & $3.41 E+03$ & $4.62 E+02$ & $1.55 E+03$ & $1.08 E+03$ & $7.45 E+03$ & $2.34 E+02$ \\
\hline $\mathrm{F} 4$ & $3.92 E+01$ & $1.18 E+00$ & $2.34 E+00$ & $2.94 E+01$ & $4.54 E+01$ & $3.48 E+01$ & $3.48 E+01$ \\
\hline F5 & $2.04 E+01$ & $2.03 E+01$ & $2.02 E+01$ & $2.02 E+01$ & $2.00 E+01$ & $2.05 E+01$ & $2.05 E+01$ \\
\hline F6 & $5.51 E+00$ & $3.78 E+00$ & $1.01 E+00$ & $3.27 E+00$ & $2.53 E+00$ & $0.00 E+00$ & $2.70 E-01$ \\
\hline F7 & $3.22 E-01$ & $6.64 E-02$ & $5.77 E-02$ & $9.71 E-01$ & $1.48 E-02$ & $2.71 E-02$ & $8.61 E-02$ \\
\hline F8 & $3.98 E+00$ & $1.49 E+01$ & $0.00 E+00$ & $6.01 E+00$ & $8.95 E+00$ & $8.95 E+00$ & $3.98 E+00$ \\
\hline F9 & $1.39 E+01$ & $1.79 E+01$ & $8.21 E+00$ & $1.89 E+01$ & $1.29 E+01$ & $7.96 E+00$ & $8.95 E+00$ \\
\hline F10 & $2.75 E+02$ & $4.50 E+02$ & $8.45 E-02$ & $1.42 E+02$ & $4.79 E+02$ & $1.71 E+02$ & $1.32 E+02$ \\
\hline F11 & $6.95 E+02$ & $7.30 E+02$ & $2.89 E+02$ & $1.20 E+03$ & $5.93 E+02$ & $3.74 E+02$ & $3.71 E+02$ \\
\hline $\mathrm{F} 12$ & $1.06 E+00$ & $2.36 E-01$ & $3.94 E-01$ & $8.10 E-01$ & $7.51 E-02$ & $1.72 E+00$ & $5.81 E-01$ \\
\hline F13 & $2.44 E-01$ & $1.90 E-01$ & $2.23 E-01$ & $3.00 E-01$ & $1.53 E-01$ & $1.09 E-01$ & $1.70 E-01$ \\
\hline F14 & $2.50 E-01$ & $2.98 E-01$ & $2.45 E-01$ & $2.77 E-01$ & $4.72 E-01$ & $4.97 E-01$ & $1.51 E-01$ \\
\hline F15 & $1.96 E+00$ & $1.38 E+00$ & $1.26 E+00$ & $2.93 E+00$ & $9.70 E-01$ & $1.43 E+00$ & $1.08 E+00$ \\
\hline F16 & $3.13 E+00$ & $3.12 E+00$ & $2.40 E+00$ & $3.20 E+00$ & $3.35 E+00$ & $3.15 E+00$ & $2.28 E+00$ \\
\hline F17 & $1.06 E+04$ & $7.54 E+03$ & $1.58 E+04$ & $7.47 E+04$ & $1.10 E+05$ & $5.00 E+04$ & $3.64 E+03$ \\
\hline F18 & $2.74 E+04$ & $2.09 E+03$ & $7.96 E+01$ & $1.07 E+04$ & $1.19 E+04$ & $1.54 E+04$ & $4.11 E+03$ \\
\hline F19 & $2.57 E+00$ & $1.92 E+00$ & $8.70 E-01$ & $3.35 E+00$ & $3.19 E+00$ & $3.03 E+00$ & $1.03 E+00$ \\
\hline F20 & $3.58 E+03$ & $1.21 E+03$ & $1.69 E+02$ & $6.13 E+03$ & $3.14 E+03$ & $1.12 E+04$ & $2.71 E+02$ \\
\hline F21 & $4.17 E+02$ & $3.91 E+03$ & $2.58 E+03$ & $4.08 E+03$ & $6.57 E+03$ & $2.11 E+04$ & $1.79 E+02$ \\
\hline F22 & $2.13 E+01$ & $2.78 E+01$ & $1.04 E+01$ & $3.75 E+01$ & $1.43 E+02$ & $3.74 E+01$ & $2.11 E+01$ \\
\hline $\mathrm{F} 23$ & $3.29 E+02$ & $3.29 E+02$ & $3.29 E+02$ & $3.30 E+02$ & $3.29 E+02$ & $3.29 E+02$ & $3.29 E+02$ \\
\hline F24 & $1.30 E+02$ & $1.28 E+02$ & $1.17 E+02$ & $1.35 E+02$ & $1.45 E+02$ & $1.17 E+02$ & $1.15 E+02$ \\
\hline F25 & $2.02 E+02$ & $2.00 E+02$ & $1.62 E+02$ & $2.01 E+02$ & $2.02 E+02$ & $2.02 E+02$ & $2.01 E+02$ \\
\hline F26 & $1.00 E+02$ & $1.00 E+02$ & $1.00 E+02$ & $1.00 E+02$ & $1.00 E+02$ & $1.00 E+02$ & $1.00 E+02$ \\
\hline F27 & $4.09 E+02$ & $4.32 E+02$ & $3.55 E+02$ & $4.02 E+02$ & $3.70 E+02$ & $3.28 E+02$ & $4.00 E+02$ \\
\hline F28 & $5.80 E+02$ & $3.06 E+02$ & $3.97 E+02$ & $6.78 E+02$ & $5.33 E+02$ & $4.87 E+02$ & $4.97 E+02$ \\
\hline F29 & $3.19 E+06$ & $2.08 E+02$ & $3.19 E+02$ & $6.00 E+02$ & $8.26 E+03$ & $8.71 E+02$ & $3.77 E+02$ \\
\hline F30 & $1.90 E+03$ & $3.37 E+02$ & $8.13 E+02$ & $1.47 E+03$ & $2.01 E+03$ & $1.09 E+03$ & $1.27 E+03$ \\
\hline
\end{tabular}



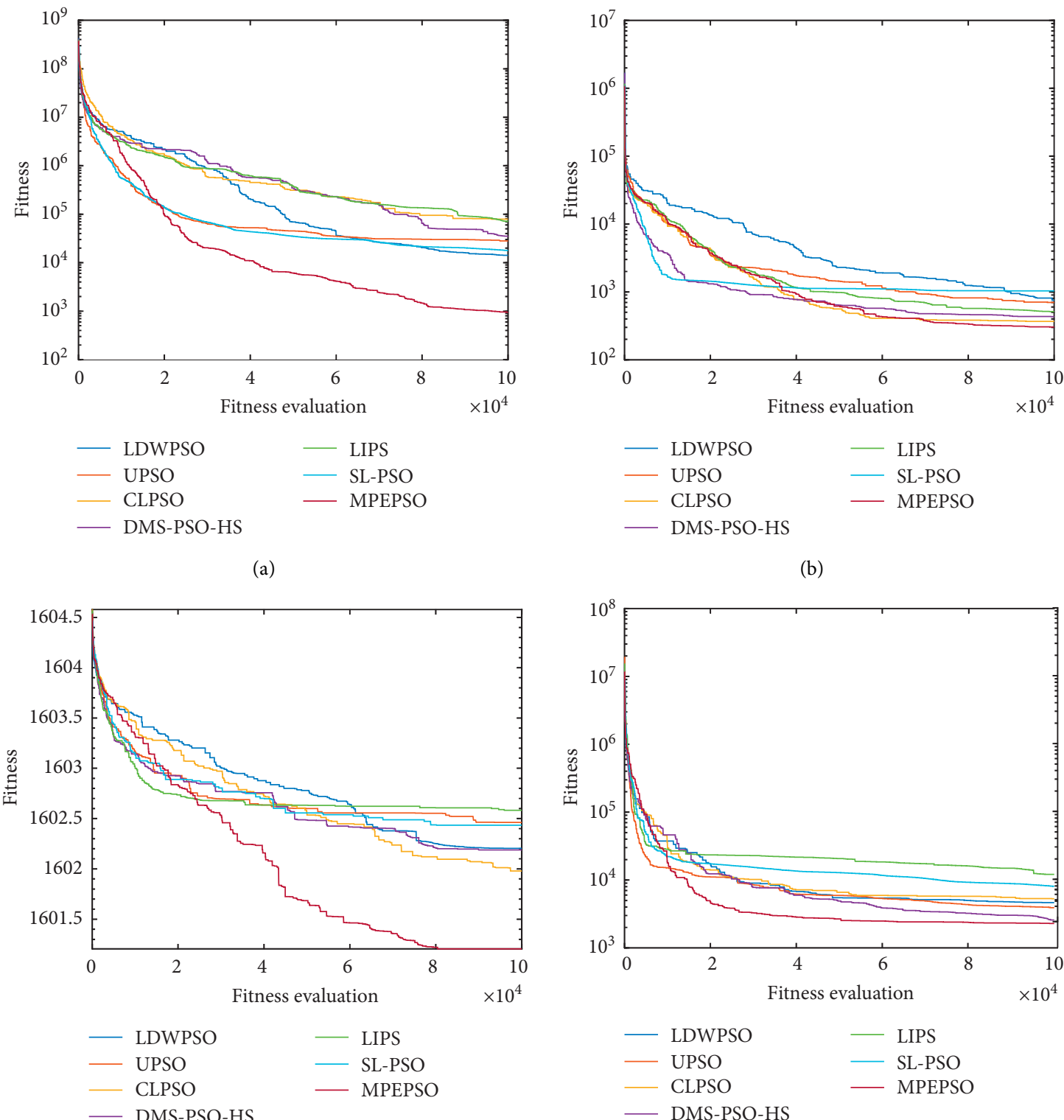

(c)
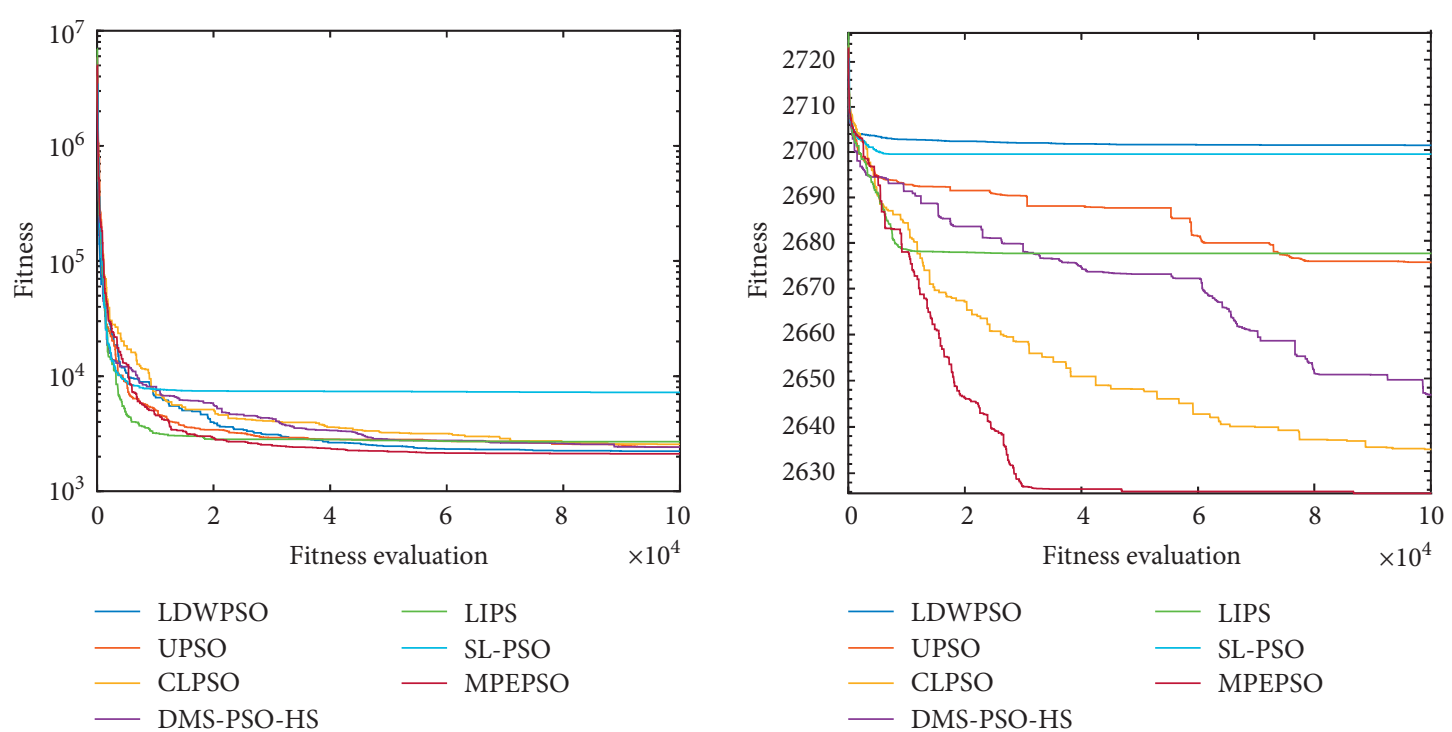

(e)

(f)

Figure 2: Median convergence graphs of six functions on 10-dimensional CEC2014 test suite: (a) F1, (b) F3, (c) F16, (d) F17, (e) F21, and (f) F25. 
TABLE 10: Computational results on 30-dimensional problems.

\begin{tabular}{|c|c|c|c|c|c|c|c|c|}
\hline & & LDWPSO & UPSO & CLPSO & DMS-PSO-HS & LIPS & SL-PSO & MPEPSO \\
\hline \multirow{3}{*}{$\mathrm{F} 1$} & Rank & 4 & 3 & 5 & 7 & 6 & 2 & 1 \\
\hline & Mean & $5.92 E+06$ & $9.93 E+05$ & $8.59 E+06$ & $4.43 E+07$ & $3.34 E+07$ & $4.27 E+05$ & $6.33 E+04$ \\
\hline & Std & $6.24 E+06$ & $6.73 E+05$ & $3.41 E+06$ & $4.96 E+07$ & $2.80 E+07$ & $2.95 E+05$ & $4.66 E+04$ \\
\hline \multirow{3}{*}{$\mathrm{F} 2$} & Rank & 3 & 5 & 2 & 7 & 4 & 6 & 1 \\
\hline & Mean & $7.54 E+01$ & $5.38 E+03$ & $3.17 E+01$ & $2.77 E+08$ & $1.78 E+03$ & $1.07 E+04$ & $2.16 E-05$ \\
\hline & Std & $1.18 E+02$ & $8.76 E+03$ & $6.54 E+01$ & $5.24 E+08$ & $4.00 E+03$ & $9.26 E+03$ & $4.62 E-05$ \\
\hline \multirow{3}{*}{ F3 } & Rank & 2 & 4 & 3 & 7 & 5 & 6 & 1 \\
\hline & Mean & $1.88 E+02$ & $5.17 E+02$ & $2.21 E+02$ & $1.01 E+04$ & $3.27 E+03$ & $6.87 E+03$ & $3.66 E-02$ \\
\hline & Std & $2.36 E+02$ & $5.68 E+02$ & $2.95 E+02$ & $1.22 E+04$ & $3.93 E+03$ & $5.97 E+03$ & $8.17 E-02$ \\
\hline \multirow{3}{*}{ F4 } & Rank & 5 & 2 & 4 & 6 & 7 & 3 & 1 \\
\hline & Mean & $1.52 E+02$ & $2.16 E+01$ & $7.12 E+01$ & $1.71 E+02$ & $2.17 E+02$ & $3.24 E+01$ & $2.47 E+00$ \\
\hline & Std & $3.35 E+01$ & $1.11 E+01$ & $1.46 E+01$ & $5.03 E+01$ & $7.69 E+01$ & $3.05 E+01$ & $1.18 E+01$ \\
\hline \multirow{3}{*}{ F5 } & Rank & 6 & 4 & 3 & 2 & 1 & 7 & 5 \\
\hline & Mean & $2.09 E+01$ & $2.04 E+01$ & $2.04 E+01$ & $2.03 E+01$ & $2.00 E+01$ & $2.09 E+01$ & $2.05 E+01$ \\
\hline & Std & $8.63 E-02$ & $1.83 E-01$ & $4.69 E-02$ & $2.41 E-01$ & $2.89 E-04$ & $4.57 E-02$ & $4.55 E-01$ \\
\hline \multirow{3}{*}{ F6 } & Rank & 3 & 7 & 4 & 6 & 5 & 1 & 2 \\
\hline & Mean & $1.17 E+01$ & $2.45 E+01$ & $1.23 E+01$ & $2.12 E+01$ & $1.36 E+01$ & $9.08 E-01$ & $5.54 E+00$ \\
\hline & Std & $2.84 E+00$ & $2.22 E+00$ & $1.21 E+00$ & $5.30 E+00$ & $2.67 E+00$ & $9.96 E-01$ & $2.30 E+00$ \\
\hline \multirow{3}{*}{ F7 } & Rank & 6 & 2 & 1 & 7 & 3 & 4 & 5 \\
\hline & Mean & $1.27 E-02$ & $1.13 E-03$ & $6.21 E-05$ & $3.44 E+00$ & $1.15 E-03$ & $1.23 E-03$ & $9.68 E-03$ \\
\hline & Std & $1.43 E-02$ & $3.90 E-03$ & $5.72 E-05$ & $4.25 E+00$ & $3.02 E-03$ & $2.80 E-03$ & $1.13 E-02$ \\
\hline \multirow{3}{*}{ F8 } & Rank & 3 & 7 & 1 & 5 & 6 & 2 & 4 \\
\hline & Mean & $1.79 E+01$ & $8.07 E+01$ & $1.17 E-13$ & $4.87 E+01$ & $5.00 E+01$ & $1.65 E+01$ & $2.01 E+01$ \\
\hline & Std & $5.45 E+00$ & $1.19 E+01$ & $2.08 E-14$ & $3.46 E+01$ & $1.44 E+01$ & $3.75 E+00$ & $7.46 E+00$ \\
\hline \multirow{3}{*}{ F9 } & Rank & 5 & 6 & 3 & 7 & 4 & 1 & 2 \\
\hline & Mean & $6.53 E+01$ & $9.38 E+01$ & $5.02 E+01$ & $9.87 E+01$ & $6.21 E+01$ & $1.77 E+01$ & $3.87 E+01$ \\
\hline & Std & $1.43 E+01$ & $2.17 E+01$ & $6.70 E+00$ & $4.07 E+01$ & $1.45 E+01$ & $9.17 E+00$ & $1.01 E+01$ \\
\hline \multirow{3}{*}{ F10 } & Rank & 4 & 7 & 1 & 5 & 6 & 2 & 3 \\
\hline & Mean & $6.09 E+02$ & $2.46 E+03$ & $5.49 E-01$ & $8.16 E+02$ & $1.90 E+03$ & $3.58 E+02$ & $4.63 E+02$ \\
\hline & Std & $2.11 E+02$ & $3.22 E+02$ & $4.07 E-01$ & $7.46 E+02$ & $3.75 E+02$ & $2.48 E+02$ & $1.86 E+02$ \\
\hline \multirow{3}{*}{ F11 } & Rank & 5 & 6 & 2 & 7 & 4 & 1 & 3 \\
\hline & Mean & $2.61 E+03$ & $3.09 E+03$ & $2.08 E+03$ & $3.83 E+03$ & $2.52 E+03$ & $9.59 E+02$ & $2.49 E+03$ \\
\hline & Std & $7.55 E+02$ & $4.29 E+02$ & $2.85 E+02$ & $1.30 E+03$ & $3.52 E+02$ & $4.78 E+02$ & $4.47 E+02$ \\
\hline \multirow{3}{*}{ F12 } & Rank & 6 & 2 & 3 & 5 & 1 & 7 & 4 \\
\hline & Mean & $1.72 E+00$ & $2.95 E-01$ & $3.22 E-01$ & $8.27 E-01$ & $1.61 E-01$ & $2.20 E+00$ & $5.01 E-01$ \\
\hline & Std & $5.08 E-01$ & $8.01 E-02$ & $5.05 E-02$ & $4.06 E-01$ & $4.66 E-02$ & $5.09 E-01$ & $6.08 E-01$ \\
\hline \multirow{3}{*}{$\mathrm{F} 13$} & Rank & 7 & 4 & 5 & 6 & 3 & 1 & 2 \\
\hline & Mean & $4.34 E-01$ & $2.91 E-01$ & $3.17 E-01$ & $3.74 E-01$ & $2.81 E-01$ & $1.68 E-01$ & $1.72 E-01$ \\
\hline & Std & $1.03 E-01$ & $6.75 E-02$ & $3.36 E-02$ & $1.02 E-01$ & $6.20 E-02$ & $3.36 E-02$ & $4.65 E-02$ \\
\hline \multirow{3}{*}{ F14 } & Rank & 6 & 4 & 3 & 5 & 2 & 7 & 1 \\
\hline & Mean & $3.21 E-01$ & $2.63 E-01$ & $2.49 E-01$ & $3.19 E-01$ & $2.36 E-01$ & $3.98 E-01$ & $2.27 E-01$ \\
\hline & Std & $1.07 E-01$ & $5.04 E-02$ & $2.62 E-02$ & $1.19 E-01$ & $4.47 E-02$ & $6.50 E-02$ & $4.38 E-02$ \\
\hline \multirow{3}{*}{ F15 } & Rank & 3 & 4 & 5 & 7 & 6 & 2 & 1 \\
\hline & Mean & $6.42 E+00$ & $7.14 E+00$ & $7.84 E+00$ & $6.35 E+01$ & $9.03 E+00$ & $4.53 E+00$ & $3.75 E+00$ \\
\hline & Std & $1.70 E+00$ & $1.84 E+00$ & $8.66 E-01$ & $1.43 E+02$ & $3.13 E+00$ & $3.56 E+00$ & $1.03 E+00$ \\
\hline \multirow{3}{*}{ F16 } & Rank & 3 & 6 & 2 & 5 & 4 & 7 & 1 \\
\hline & Mean & $1.11 E+01$ & $1.18 E+01$ & $1.00 E+01$ & $1.16 E+01$ & $1.15 E+01$ & $1.21 E+01$ & $9.65 E+00$ \\
\hline & Std & $8.70 E-01$ & $3.40 E-01$ & $3.42 E-01$ & $8.05 E-01$ & $5.27 E-01$ & $3.58 E-01$ & $7.22 E-01$ \\
\hline \multirow{3}{*}{ F17 } & Rank & 6 & 3 & 7 & 5 & 4 & 2 & 1 \\
\hline & Mean & $5.79 E+05$ & $1.51 E+05$ & $1.07 E+06$ & $5.68 E+05$ & $1.81 E+05$ & $9.67 E+04$ & $2.35 E+04$ \\
\hline & Std & $5.08 E+05$ & $8.13 E+04$ & $5.39 E+05$ & $5.98 E+05$ & $1.31 E+05$ & $6.60 E+04$ & $1.72 E+04$ \\
\hline & Rank & 6 & 3 & 1 & 7 & 2 & 5 & 4 \\
\hline F18 & Mean & $2.01 E+04$ & $1.10 E+03$ & $7.20 E+01$ & $1.81 E+05$ & $4.00 E+02$ & $1.95 E+03$ & $1.44 E+03$ \\
\hline & Std & $6.99 E+04$ & $1.05 E+03$ & $2.57 E+01$ & $8.71 E+05$ & $7.62 E+02$ & $2.33 E+03$ & $2.24 E+03$ \\
\hline
\end{tabular}


Table 10: Continued.

\begin{tabular}{|c|c|c|c|c|c|c|c|c|}
\hline & & LDWPSO & UPSO & CLPSO & DMS-PSO-HS & LIPS & SL-PSO & MPEPSO \\
\hline \multirow{3}{*}{ F19 } & Rank & 4 & 5 & 3 & 6 & 7 & 2 & 1 \\
\hline & Mean & $8.31 E+00$ & $1.12 E+01$ & $7.47 E+00$ & $1.64 E+01$ & $2.64 E+01$ & $6.81 E+00$ & $4.29 E+00$ \\
\hline & Std & $2.70 E+00$ & $2.51 E+00$ & $7.01 E-01$ & $1.31 E+01$ & $2.08 E+01$ & $9.04 E-01$ & $1.24 E+00$ \\
\hline \multirow{3}{*}{ F20 } & Rank & 2 & 4 & 3 & 5 & 6 & 7 & 1 \\
\hline & Mean & $4.93 E+02$ & $4.80 E+03$ & $3.55 E+03$ & $4.94 E+03$ & $1.35 E+04$ & $2.00 E+04$ & $1.99 E+02$ \\
\hline & Std & $2.19 E+02$ & $3.13 E+03$ & $1.91 E+03$ & $3.55 E+03$ & $7.07 E+03$ & $1.52 E+04$ & $1.20 E+02$ \\
\hline \multirow{3}{*}{$\mathrm{F} 21$} & Rank & 6 & 2 & 5 & 7 & 4 & 3 & 1 \\
\hline & Mean & $1.24 E+05$ & $5.24 E+04$ & $8.72 E+04$ & $3.38 E+05$ & $7.72 E+04$ & $7.53 E+04$ & $1.08 E+04$ \\
\hline & Std & $1.37 E+05$ & $4.33 E+04$ & $5.35 E+04$ & $5.17 E+05$ & $6.06 E+04$ & $6.20 E+04$ & $7.04 E+03$ \\
\hline \multirow{3}{*}{ F22 } & Rank & 4 & 6 & 3 & 7 & 5 & 2 & 1 \\
\hline & Mean & $2.52 E+02$ & $3.00 E+02$ & $2.02 E+02$ & $3.93 E+02$ & $2.73 E+02$ & $1.47 E+02$ & $1.46 E+02$ \\
\hline & Std & $1.16 E+02$ & $1.17 E+02$ & $7.30 E+01$ & $1.21 E+02$ & $1.14 E+02$ & $8.72 E+01$ & $5.08 E+01$ \\
\hline \multirow{3}{*}{ F23 } & Rank & 5 & 1 & 4 & 6 & 7 & 2 & 2 \\
\hline & Mean & $3.16 E+02$ & $3.14 E+02$ & $3.15 E+02$ & $3.19 E+02$ & $3.23 E+02$ & $3.15 E+02$ & $3.15 E+02$ \\
\hline & Std & $1.85 E-01$ & $0.00 E+00$ & $2.06 E-04$ & $5.50 E+00$ & $5.16 E+00$ & $1.51 E-12$ & $5.72 E-13$ \\
\hline \multirow{3}{*}{ F24 } & Rank & 5 & 3 & 1 & 7 & 6 & 4 & 2 \\
\hline & Mean & $2.32 E+02$ & $2.29 E+02$ & $2.24 E+02$ & $2.39 E+02$ & $2.35 E+02$ & $2.31 E+02$ & $2.25 E+02$ \\
\hline & Std & $6.07 E+00$ & $5.60 E+00$ & $4.78 E+00$ & $8.09 E+00$ & $5.76 E+00$ & $5.82 E+00$ & $2.65 E+00$ \\
\hline \multirow{3}{*}{ F25 } & Rank & 5 & 1 & 4 & 7 & 6 & 2 & 3 \\
\hline & Mean & $2.08 E+02$ & $2.01 E+02$ & $2.08 E+02$ & $2.19 E+02$ & $2.15 E+02$ & $2.05 E+02$ & $2.08 E+02$ \\
\hline & Std & $1.92 E+00$ & $2.17 E-01$ & $7.72 E-01$ & $6.06 E+00$ & $2.69 E+00$ & $1.31 E+00$ & $2.39 E+00$ \\
\hline \multirow{3}{*}{ F26 } & Rank & 5 & 1 & 2 & 7 & 6 & 4 & 3 \\
\hline & Mean & $1.14 E+02$ & $1.00 E+02$ & $1.00 E+02$ & $1.30 E+02$ & $1.14 E+02$ & $1.14 E+02$ & $1.07 E+02$ \\
\hline & Std & $3.45 E+01$ & $7.25 E-02$ & $7.26 E-02$ & $4.58 E+01$ & $3.14 E+01$ & $3.45 E+01$ & $2.53 E+01$ \\
\hline \multirow{3}{*}{ F27 } & Rank & 6 & 7 & 3 & 4 & 5 & 1 & 2 \\
\hline & Mean & $5.85 E+02$ & $8.54 E+02$ & $4.12 E+02$ & $4.94 E+02$ & $5.06 E+02$ & $3.73 E+02$ & $4.11 E+02$ \\
\hline & Std & $1.05 E+02$ & $1.99 E+02$ & $4.28 E+00$ & $1.25 E+02$ & $1.17 E+02$ & $4.97 E+01$ & $4.93 E+01$ \\
\hline \multirow{3}{*}{ F28 } & Rank & 5 & 1 & 2 & 6 & 7 & 4 & 3 \\
\hline & Mean & $1.06 E+03$ & $4.27 E+02$ & $8.89 E+02$ & $1.55 E+03$ & $1.63 E+03$ & $9.28 E+02$ & $9.26 E+02$ \\
\hline & Std & $1.88 E+02$ & $2.40 E+01$ & $3.70 E+01$ & $6.55 E+02$ & $4.82 E+02$ & $1.19 E+02$ & $5.51 E+01$ \\
\hline \multirow{3}{*}{ F29 } & Rank & 7 & 1 & 2 & 6 & 5 & 4 & 3 \\
\hline & Mean & $2.39 E+06$ & $2.11 E+02$ & $9.21 E+02$ & $3.07 E+04$ & $2.59 E+03$ & $1.72 E+03$ & $1.07 E+03$ \\
\hline & Std & $7.37 E+06$ & $2.76 E+00$ & $8.56 E+01$ & $5.86 E+04$ & $5.81 E+03$ & $4.88 E+02$ & $2.56 E+02$ \\
\hline \multirow{3}{*}{ F30 } & Rank & 5 & 1 & 4 & 6 & 7 & 3 & 2 \\
\hline & Mean & $3.81 E+03$ & $6.61 E+02$ & $3.60 E+03$ & $1.62 E+04$ & $3.67 E+04$ & $3.17 E+03$ & $1.85 E+03$ \\
\hline & Std & $1.16 E+03$ & $1.78 E+02$ & $9.01 E+02$ & $2.18 E+04$ & $1.98 E+04$ & $1.03 E+03$ & $5.08 E+02$ \\
\hline
\end{tabular}

dimensions. The rank of each algorithm, mean error, and standard deviation values on 10-dimensional problems are shown in Table 20. The results of Wilcoxon signed-rank tests are shown in Table 21.

For unimodal functions (F1 F3), L-SHADE provides the best solutions on all functions. MPEPSO gives the second-best solutions on functions $\mathrm{F} 1$ and F2. For simple multimodal functions (F4 F16), MPEPSO ranks first for function F14 and ranks second for functions F4 and F16. For hybrid functions (F17 F22), MPEPSO gives the second-best solutions on function F19 and F22, while L-SHADE provides the best solutions on all the functions. For composition functions (F23 F30), MPEPSO performs best on function F23. MPEPSO has the best performance on 2 functions and the second-best performance on 8 functions out of 30 functions.

As shown in Table 21, according to the results of Wilcoxon signed-rank tests, MPEPSO is significantly better than CS, FA, SCA, GCMBO, and L-SHADE on 16, 17, 28, 28, and 0 functions, respectively. It is inferior to CS, FA, SCA, GCMBO, and L-SHADE on 11, 10, 2, 2, and 27 functions while similar to them on $3,3,0,0$, and 3 functions, respectively.

As shown in Table 22, the average rank of MPEPSO is 2.5. L-SHADE ranks first for the CEC 2014 test suite on 30dimensional problems.

4.4. Computational Time. According to the instructions given in [41], the computational time of the algorithms is compared on 10 and 30 dimensions. This method is widely used to evaluate the algorithm complexity, such as [34, 53-55].

The computational time is calculated as follows:

(1) Execute the test program that described in [41] to obtain the computing time $T_{0}$.

(2) Record the computing time for 200,000 evaluations of F18 in CEC 2014 test suite. The computing time is represented by $T_{1}$. 
TABLE 11: Wilcoxon signed-rank test for 30 runs on 30-dimensional problems.

\begin{tabular}{|c|c|c|c|c|c|c|}
\hline & LDWPSO & UPSO & CLPSO & DMS-PSO-HS & LIPS & SL-PSO \\
\hline F1 & + & + & + & + & + & + \\
\hline $\mathrm{F} 2$ & + & + & + & + & + & + \\
\hline F3 & + & + & + & + & + & + \\
\hline $\mathrm{F} 4$ & + & + & + & + & + & + \\
\hline F5 & + & $=$ & - & - & - & + \\
\hline F6 & + & + & + & + & + & - \\
\hline F7 & $=$ & - & - & + & - & - \\
\hline F8 & $=$ & + & - & + & + & $=$ \\
\hline F9 & + & + & + & + & + & - \\
\hline F10 & + & + & - & + & + & - \\
\hline F11 & $=$ & + & - & + & $=$ & - \\
\hline F12 & + & $=$ & $=$ & + & - & + \\
\hline F13 & + & + & + & + & + & $=$ \\
\hline F14 & + & + & $=$ & + & $=$ & + \\
\hline F15 & + & + & + & + & + & $=$ \\
\hline F16 & + & + & + & + & + & + \\
\hline F17 & + & + & + & + & + & + \\
\hline F18 & + & $=$ & - & $=$ & $=$ & $=$ \\
\hline F19 & + & + & + & + & + & + \\
\hline F20 & + & + & + & + & + & + \\
\hline F21 & + & + & + & + & + & + \\
\hline F22 & + & + & + & + & + & $=$ \\
\hline F23 & + & - & + & + & + & + \\
\hline F24 & + & + & $=$ & + & + & + \\
\hline F25 & $=$ & - & $=$ & + & + & - \\
\hline F26 & + & - & - & + & + & $=$ \\
\hline F27 & + & + & + & + & + & - \\
\hline F28 & + & - & - & + & + & $=$ \\
\hline F29 & + & - & - & + & + & + \\
\hline F30 & + & - & + & + & + & + \\
\hline$+1=1-$ & $26 / 4 / 0$ & $20 / 3 / 7$ & $17 / 4 / 9$ & $28 / 1 / 1$ & $24 / 3 / 3$ & $16 / 7 / 7$ \\
\hline
\end{tabular}

TABLE 12: The $p$ value and $h$ of the Wilcoxon signed-rank test on 30-dimensional problems.

\begin{tabular}{|c|c|c|c|c|c|c|c|c|c|c|c|c|}
\hline & \multicolumn{2}{|c|}{ LDWPSO } & \multicolumn{2}{|c|}{ UPSO } & \multicolumn{2}{|c|}{ CLPSO } & \multicolumn{2}{|c|}{ DMS-PSO-HS } & \multicolumn{2}{|l|}{ LIPS } & \multicolumn{2}{|c|}{ SL-PSO } \\
\hline & $p$ value & $h$ & $p$ value & $h$ & $p$ value & $h$ & $p$ value & $h$ & $p$ value & $h$ & $p$ value & $h$ \\
\hline F1 & $1.73 E-06$ & 1 & $1.73 E-06$ & 1 & $1.73 E-06$ & 1 & $1.73 E-06$ & 1 & $1.73 E-06$ & 1 & $1.92 E-06$ & 1 \\
\hline F2 & $1.73 E-06$ & 1 & $1.73 E-06$ & 1 & $1.73 E-06$ & 1 & $1.73 E-06$ & 1 & $1.73 E-06$ & 1 & $1.73 E-06$ & 1 \\
\hline F3 & $1.73 E-06$ & 1 & $1.73 E-06$ & 1 & $1.73 E-06$ & 1 & $1.73 E-06$ & 1 & $1.73 E-06$ & 1 & $1.73 E-06$ & 1 \\
\hline F4 & $1.73 E-06$ & 1 & $2.84 E-05$ & 1 & $1.73 E-06$ & 1 & $1.73 E-06$ & 1 & $1.73 E-06$ & 1 & $8.47 E-06$ & 1 \\
\hline F5 & $4.68 E-03$ & 1 & $9.37 E-02$ & 0 & $4.39 E-03$ & 1 & $2.18 E-02$ & 1 & $2.60 E-06$ & 1 & $3.41 E-05$ & 1 \\
\hline F6 & $2.35 E-06$ & 1 & $1.73 E-06$ & 1 & $1.73 E-06$ & 1 & $1.73 E-06$ & 1 & $1.73 E-06$ & 1 & $1.92 E-06$ & 1 \\
\hline F7 & $4.65 E-01$ & 0 & $4.23 E-04$ & 1 & $2.07 E-02$ & 1 & $1.92 E-06$ & 1 & $3.00 E-02$ & 1 & $2.31 E-04$ & 1 \\
\hline F8 & $3.39 E-01$ & 0 & $1.73 E-06$ & 1 & $1.73 E-06$ & 1 & $6.32 E-05$ & 1 & $2.13 E-06$ & 1 & $5.29 E-02$ & 0 \\
\hline F9 & $4.73 E-06$ & 1 & $1.92 E-06$ & 1 & $2.61 E-04$ & 1 & $2.35 E-06$ & 1 & $3.52 E-06$ & 1 & $2.60 E-06$ & 1 \\
\hline F10 & $4.99 E-03$ & 1 & $1.73 E-06$ & 1 & $1.73 E-06$ & 1 & $3.68 E-02$ & 1 & $1.73 E-06$ & 1 & $4.95 E-02$ & 1 \\
\hline F11 & $6.14 E-01$ & 0 & $3.41 E-05$ & 1 & $4.53 E-04$ & 1 & $2.37 E-05$ & 1 & $4.78 E-01$ & 0 & $1.73 E-06$ & 1 \\
\hline F12 & $4.86 E-05$ & 1 & $6.44 E-01$ & 0 & $9.75 E-01$ & 0 & $2.58 E-03$ & 1 & $5.22 E-06$ & 1 & $2.88 E-06$ & 1 \\
\hline F13 & $1.73 E-06$ & 1 & $3.52 E-06$ & 1 & $1.73 E-06$ & 1 & $1.73 E-06$ & 1 & $1.36 E-05$ & 1 & $7.81 E-01$ & 0 \\
\hline F14 & $2.60 E-05$ & 1 & $7.27 E-03$ & 1 & $6.56 E-02$ & 0 & $8.19 E-05$ & 1 & $3.39 E-01$ & 0 & $1.73 E-06$ & 1 \\
\hline F15 & $7.69 E-06$ & 1 & $1.73 E-06$ & 1 & $1.92 E-06$ & 1 & $1.73 E-06$ & 1 & $1.73 E-06$ & 1 & $6.44 E-01$ & 0 \\
\hline F16 & $1.92 E-06$ & 1 & $1.73 E-06$ & 1 & $2.43 E-02$ & 1 & $2.35 E-06$ & 1 & $1.73 E-06$ & 1 & $1.73 E-06$ & 1 \\
\hline F17 & $2.13 E-06$ & 1 & $1.73 E-06$ & 1 & $1.73 E-06$ & 1 & $1.73 E-06$ & 1 & $2.35 E-06$ & 1 & $1.24 E-05$ & 1 \\
\hline F18 & $2.16 E-05$ & 1 & $9.75 E-01$ & 0 & $3.11 E-05$ & 1 & $2.06 E-01$ & 0 & $6.27 E-02$ & 0 & $2.13 E-01$ & 0 \\
\hline F19 & $1.92 E-06$ & 1 & $1.73 E-06$ & 1 & $2.13 E-06$ & 1 & $1.73 E-06$ & 1 & $1.73 E-06$ & 1 & $5.22 E-06$ & 1 \\
\hline F20 & $1.97 E-05$ & 1 & $1.73 E-06$ & 1 & $1.73 E-06$ & 1 & $1.73 E-06$ & 1 & $1.73 E-06$ & 1 & $1.73 E-06$ & 1 \\
\hline F21 & $2.35 E-06$ & 1 & $2.60 E-06$ & 1 & $1.73 E-06$ & 1 & $1.73 E-06$ & 1 & $7.69 E-06$ & 1 & $1.92 E-06$ & 1 \\
\hline F22 & $2.83 E-04$ & 1 & $3.11 E-05$ & 1 & $1.59 E-03$ & 1 & $1.73 E-06$ & 1 & $3.41 E-05$ & 1 & $5.72 E-01$ & 0 \\
\hline F23 & $1.73 E-06$ & 1 & $1.25 E-06$ & 1 & $1.73 E-06$ & 1 & $2.88 E-06$ & 1 & $1.73 E-06$ & 1 & $5.23 E-06$ & 1 \\
\hline
\end{tabular}


TABle 12: Continued.

\begin{tabular}{|c|c|c|c|c|c|c|c|c|c|c|c|c|}
\hline & \multicolumn{2}{|c|}{ LDWPSO } & \multicolumn{2}{|c|}{ UPSO } & \multicolumn{2}{|c|}{ CLPSO } & \multicolumn{2}{|c|}{ DMS-PSO-HS } & \multicolumn{2}{|c|}{ LIPS } & \multicolumn{2}{|c|}{ SL-PSO } \\
\hline & $p$ value & $h$ & $p$ value & $h$ & $p$ value & $h$ & $p$ value & $h$ & $p$ value & $h$ & $p$ value & $h$ \\
\hline F24 & $1.25 E-04$ & 1 & $4.53 E-04$ & 1 & $3.60 E-01$ & 0 & $1.73 E-06$ & 1 & $3.88 E-06$ & 1 & $1.36 E-05$ & 1 \\
\hline F25 & $7.86 E-02$ & 0 & $1.73 E-06$ & 1 & $7.97 E-01$ & 0 & $1.92 E-06$ & 1 & $1.73 E-06$ & 1 & $1.11 E-03$ & 1 \\
\hline F26 & $1.89 E-04$ & 1 & $3.88 E-04$ & 1 & $3.59 E-04$ & 1 & $1.64 E-05$ & 1 & $2.22 E-04$ & 1 & $1.71 E-01$ & 0 \\
\hline F27 & $3.18 E-06$ & 1 & $1.73 E-06$ & 1 & $1.11 E-02$ & 1 & $1.60 E-04$ & 1 & $1.36 E-04$ & 1 & $4.99 E-03$ & 1 \\
\hline F28 & $1.89 E-04$ & 1 & $1.73 E-06$ & 1 & $1.71 E-03$ & 1 & $3.52 E-06$ & 1 & $1.73 E-06$ & 1 & $2.29 E-01$ & 0 \\
\hline F29 & $4.68 E-03$ & 1 & $1.73 E-06$ & 1 & $2.11 E-03$ & 1 & $3.72 E-05$ & 1 & $8.31 E-04$ & 1 & $7.69 E-06$ & 1 \\
\hline F30 & $3.18 E-06$ & 1 & $1.73 E-06$ & 1 & $2.60 E-06$ & 1 & $2.60 E-06$ & 1 & $1.73 E-06$ & 1 & $5.79 E-05$ & 1 \\
\hline
\end{tabular}

TABLE 13: The rank of the seven algorithms on 30-dimensional problems.

\begin{tabular}{lccccccc}
\hline Rank & LDWPSO & UPSO & CLPSO & DMS-PSO-HS & LIPS & SL-PSO & MPEPSO \\
\hline Total rank & 142 & 112 & 91 & 180 & 144 & 104 \\
Ave. rank & 4.733333 & 3.733333 & 3.033333 & 6 & 4.8 & 3.466667 & 2.2 \\
Final rank & 5 & 4 & 2 & 7 & 6 & 3 \\
\hline
\end{tabular}

TABLE 14: Best values of function error values for 30 runs on 30-dimensional problems.

\begin{tabular}{|c|c|c|c|c|c|c|c|}
\hline & LDWPSO & UPSO & CLPSO & DMS-PSO-HS & LIPS & SL-PSO & MPEPSO \\
\hline $\mathrm{F} 1$ & $4.85 E+05$ & $2.82 E+05$ & $1.59 E+06$ & $1.36 E+06$ & $3.87 E+05$ & $8.75 E+04$ & $1.06 E+04$ \\
\hline $\mathrm{F} 2$ & $3.50 E-03$ & $1.88 E-02$ & $4.89 E-02$ & $1.10 E+04$ & $1.14 E-01$ & $1.23 E+02$ & $7.07 E-11$ \\
\hline F3 & $2.21 E+00$ & $9.11 E+00$ & $4.54 E+00$ & $3.28 E+00$ & $1.23 E+02$ & $1.34 E+02$ & $3.57 E-06$ \\
\hline $\mathrm{F} 4$ & $7.74 E+01$ & $1.43 E+00$ & $3.44 E+01$ & $7.60 E+01$ & $7.54 E+01$ & $2.58 E+00$ & $1.39 E-03$ \\
\hline F5 & $2.06 E+01$ & $2.01 E+01$ & $2.02 E+01$ & $2.00 E+01$ & $2.00 E+01$ & $2.08 E+01$ & $2.00 E+01$ \\
\hline F6 & $7.80 E+00$ & $2.01 E+01$ & $1.02 E+01$ & $1.27 E+01$ & $7.44 E+00$ & $0.00 E+00$ & $2.11 E+00$ \\
\hline F7 & $3.41 E-13$ & $0.00 E+00$ & $3.46 E-06$ & $8.49 E-03$ & $6.82 E-13$ & $1.14 E-13$ & $1.14 E-13$ \\
\hline F8 & $8.95 E+00$ & $5.67 E+01$ & $1.14 E-13$ & $3.99 E+00$ & $2.79 E+01$ & $9.95 E+00$ & $8.95 E+00$ \\
\hline F9 & $3.98 E+01$ & $5.87 E+01$ & $3.56 E+01$ & $3.57 E+01$ & $3.68 E+01$ & $4.97 E+00$ & $2.29 E+01$ \\
\hline F10 & $1.37 E+02$ & $1.86 E+03$ & $1.79 E-01$ & $9.37 E+00$ & $1.25 E+03$ & $1.29 E+01$ & $1.25 E+02$ \\
\hline F11 & $1.73 E+03$ & $2.07 E+03$ & $1.55 E+03$ & $1.73 E+03$ & $1.78 E+03$ & $2.59 E+02$ & $1.65 E+03$ \\
\hline F12 & $3.13 E-01$ & $1.27 E-01$ & $2.11 E-01$ & $3.26 E-01$ & $7.32 E-02$ & $5.52 E-01$ & $1.56 E-01$ \\
\hline F13 & $2.27 E-01$ & $1.59 E-01$ & $2.60 E-01$ & $2.31 E-01$ & $1.38 E-01$ & $1.00 E-01$ & $8.65 E-02$ \\
\hline F14 & $1.83 E-01$ & $1.34 E-01$ & $1.86 E-01$ & $1.43 E-01$ & $1.45 E-01$ & $2.38 E-01$ & $1.39 E-01$ \\
\hline F15 & $3.59 E+00$ & $4.77 E+00$ & $6.17 E+00$ & $8.49 E+00$ & $4.62 E+00$ & $1.89 E+00$ & $1.99 E+00$ \\
\hline F16 & $8.52 E+00$ & $1.09 E+01$ & $9.39 E+00$ & $9.56 E+00$ & $1.04 E+01$ & $1.11 E+01$ & $7.78 E+00$ \\
\hline F17 & $3.70 E+04$ & $4.14 E+04$ & $1.89 E+05$ & $8.41 E+04$ & $2.18 E+04$ & $1.17 E+04$ & $4.57 E+03$ \\
\hline F18 & $3.88 E+02$ & $1.48 E+02$ & $3.49 E+01$ & $5.80 E+01$ & $7.53 E+01$ & $3.56 E+01$ & $2.02 E+01$ \\
\hline F19 & $4.58 E+00$ & $6.24 E+00$ & $6.04 E+00$ & $7.87 E+00$ & $5.25 E+00$ & $4.96 E+00$ & $2.10 E+00$ \\
\hline F20 & $2.02 E+02$ & $7.91 E+02$ & $1.17 E+03$ & $9.12 E+02$ & $1.60 E+03$ & $4.04 E+03$ & $6.51 E+01$ \\
\hline F21 & $9.01 E+03$ & $8.90 E+03$ & $2.15 E+04$ & $4.03 E+04$ & $3.41 E+03$ & $6.21 E+03$ & $1.15 E+03$ \\
\hline F22 & $2.29 E+01$ & $3.85 E+01$ & $2.47 E+01$ & $1.65 E+02$ & $3.83 E+01$ & $2.16 E+01$ & $2.35 E+01$ \\
\hline F23 & $3.16 E+02$ & $3.14 E+02$ & $3.15 E+02$ & $3.15 E+02$ & $3.17 E+02$ & $3.15 E+02$ & $3.15 E+02$ \\
\hline F24 & $2.24 E+02$ & $2.24 E+02$ & $2.02 E+02$ & $2.27 E+02$ & $2.26 E+02$ & $2.23 E+02$ & $2.23 E+02$ \\
\hline F25 & $2.06 E+02$ & $2.00 E+02$ & $2.06 E+02$ & $2.06 E+02$ & $2.09 E+02$ & $2.03 E+02$ & $2.03 E+02$ \\
\hline F26 & $1.00 E+02$ & $1.00 E+02$ & $1.00 E+02$ & $1.00 E+02$ & $1.00 E+02$ & $1.00 E+02$ & $1.00 E+02$ \\
\hline F27 & $4.02 E+02$ & $4.04 E+02$ & $4.04 E+02$ & $4.03 E+02$ & $4.05 E+02$ & $3.00 E+02$ & $3.26 E+02$ \\
\hline F28 & $8.32 E+02$ & $3.99 E+02$ & $8.24 E+02$ & $9.16 E+02$ & $1.02 E+03$ & $7.81 E+02$ & $8.33 E+02$ \\
\hline F29 & $6.35 E+02$ & $2.06 E+02$ & $7.96 E+02$ & $7.58 E+02$ & $7.14 E+02$ & $1.07 E+03$ & $6.99 E+02$ \\
\hline F30 & $1.50 E+03$ & $3.18 E+02$ & $1.74 E+03$ & $7.21 E+02$ & $7.22 E+03$ & $1.36 E+03$ & $7.06 E+02$ \\
\hline
\end{tabular}

(3) The complete computing time for the algorithm with 200,000 evaluations of F18 is $T_{2}$.

(4) Execute step 3 five times to calculate the mean value of $T_{2}$, which is recorded as $\widehat{T}_{2}$.

The computational time of the algorithms is evaluated by $\left(\widehat{T}_{2}-T_{1}\right) / T_{0}$. The computational times of seven algorithms on 10 and 30 dimensions are shown in Tables 23 and 24, respectively. The computational time of each algorithm is ranked based on the value of $\left(\widehat{T}_{2}-T_{1}\right) / T_{0}$. The computational time of MPEPSO ranks third in both experiments on 10 and 30 dimensions. The computational cost of MPEPSO is higher than that of SL-PSO and LDWPSO, while lower than that of other algorithms. UPSO 
TABLE 15: Worst values of function error values for 30 runs on 30-dimensional problems.

\begin{tabular}{|c|c|c|c|c|c|c|c|}
\hline & LDWPSO & UPSO & CLPSO & DMS-PSO-HS & LIPS & SL-PSO & MPEPSO \\
\hline $\mathrm{F} 1$ & $2.47 E+07$ & $2.89 E+06$ & $1.66 E+07$ & $1.91 E+08$ & $8.49 E+07$ & $1.24 E+06$ & $1.91 E+05$ \\
\hline F2 & $4.17 E+02$ & $4.30 E+04$ & $3.47 E+02$ & $1.74 E+09$ & $1.75 E+04$ & $3.29 E+04$ & $2.08 E-04$ \\
\hline F3 & $8.82 E+02$ & $2.27 E+03$ & $1.23 E+03$ & $5.96 E+04$ & $1.83 E+04$ & $2.53 E+04$ & $4.10 E-01$ \\
\hline $\mathrm{F} 4$ & $2.11 E+02$ & $7.41 E+01$ & $8.90 E+01$ & $2.89 E+02$ & $3.75 E+02$ & $8.20 E+01$ & $6.50 E+01$ \\
\hline F5 & $2.10 E+01$ & $2.08 E+01$ & $2.04 E+01$ & $2.09 E+01$ & $2.00 E+01$ & $2.10 E+01$ & $2.10 E+01$ \\
\hline F6 & $1.88 E+01$ & $2.89 E+01$ & $1.45 E+01$ & $3.00 E+01$ & $1.86 E+01$ & $3.52 E+00$ & $1.22 E+01$ \\
\hline F7 & $6.39 E-02$ & $1.92 E-02$ & $2.44 E-04$ & $1.89 E+01$ & $9.86 E-03$ & $7.40 E-03$ & $3.19 E-02$ \\
\hline F8 & $3.38 E+01$ & $1.11 E+02$ & $2.27 E-13$ & $1.37 E+02$ & $9.05 E+01$ & $2.40 E+01$ & $3.58 E+01$ \\
\hline F9 & $8.95 E+01$ & $1.43 E+02$ & $6.27 E+01$ & $2.08 E+02$ & $9.15 E+01$ & $6.04 E+01$ & $6.47 E+01$ \\
\hline F10 & $9.65 E+02$ & $3.03 E+03$ & $1.46 E+00$ & $3.27 E+03$ & $2.66 E+03$ & $9.83 E+02$ & $7.84 E+02$ \\
\hline F11 & $5.55 E+03$ & $3.69 E+03$ & $2.56 E+03$ & $6.73 E+03$ & $3.19 E+03$ & $2.04 E+03$ & $3.49 E+03$ \\
\hline F12 & $2.34 E+00$ & $4.24 E-01$ & $4.01 E-01$ & $1.75 E+00$ & $2.72 E-01$ & $2.74 E+00$ & $2.39 E+00$ \\
\hline $\mathrm{F} 13$ & $6.23 E-01$ & $4.65 E-01$ & $4.01 E-01$ & $6.29 E-01$ & $4.24 E-01$ & $2.33 E-01$ & $2.70 E-01$ \\
\hline F14 & $7.08 E-01$ & $3.75 E-01$ & $2.97 E-01$ & $7.30 E-01$ & $3.18 E-01$ & $4.97 E-01$ & $3.01 E-01$ \\
\hline F15 & $9.73 E+00$ & $1.27 E+01$ & $9.42 E+00$ & $7.44 E+02$ & $1.84 E+01$ & $1.38 E+01$ & $7.19 E+00$ \\
\hline F16 & $1.21 E+01$ & $1.24 E+01$ & $1.08 E+01$ & $1.27 E+01$ & $1.23 E+01$ & $1.27 E+01$ & $1.09 E+01$ \\
\hline F17 & $2.17 E+06$ & $3.74 E+05$ & $2.60 E+06$ & $2.50 E+06$ & $4.71 E+05$ & $2.31 E+05$ & $7.52 E+04$ \\
\hline F18 & $3.88 E+05$ & $4.70 E+03$ & $1.26 E+02$ & $4.77 E+06$ & $4.32 E+03$ & $9.29 E+03$ & $1.05 E+04$ \\
\hline F19 & $1.69 E+01$ & $1.52 E+01$ & $8.79 E+00$ & $6.81 E+01$ & $8.76 E+01$ & $8.82 E+00$ & $6.82 E+00$ \\
\hline F20 & $1.22 E+03$ & $1.27 E+04$ & $9.15 E+03$ & $1.77 E+04$ & $3.09 E+04$ & $5.71 E+04$ & $5.55 E+02$ \\
\hline $\mathrm{F} 21$ & $6.70 E+05$ & $2.30 E+05$ & $2.40 E+05$ & $2.70 E+06$ & $2.66 E+05$ & $2.49 E+05$ & $2.86 E+04$ \\
\hline F22 & $5.46 E+02$ & $4.90 E+02$ & $3.59 E+02$ & $6.46 E+02$ & $4.42 E+02$ & $3.38 E+02$ & $2.68 E+02$ \\
\hline F23 & $3.16 E+02$ & $3.14 E+02$ & $3.15 E+02$ & $3.38 E+02$ & $3.36 E+02$ & $3.15 E+02$ & $3.15 E+02$ \\
\hline F24 & $2.43 E+02$ & $2.45 E+02$ & $2.27 E+02$ & $2.59 E+02$ & $2.44 E+02$ & $2.42 E+02$ & $2.35 E+02$ \\
\hline F25 & $2.13 E+02$ & $2.01 E+02$ & $2.09 E+02$ & $2.31 E+02$ & $2.21 E+02$ & $2.09 E+02$ & $2.12 E+02$ \\
\hline F26 & $2.00 E+02$ & $1.00 E+02$ & $1.01 E+02$ & $2.02 E+02$ & $2.00 E+02$ & $2.00 E+02$ & $2.00 E+02$ \\
\hline F27 & $7.61 E+02$ & $1.03 E+03$ & $4.21 E+02$ & $7.97 E+02$ & $8.91 E+02$ & $4.54 E+02$ & $6.31 E+02$ \\
\hline F28 & $1.66 E+03$ & $5.21 E+02$ & $9.62 E+02$ & $2.95 E+03$ & $2.88 E+03$ & $1.42 E+03$ & $1.12 E+03$ \\
\hline F29 & $2.71 E+07$ & $2.16 E+02$ & $1.09 E+03$ & $2.93 E+05$ & $3.25 E+04$ & $2.76 E+03$ & $1.81 E+03$ \\
\hline F30 & $5.65 E+03$ & $1.01 E+03$ & $6.06 E+03$ & $1.00 E+05$ & $8.99 E+04$ & $5.18 E+03$ & $2.89 E+03$ \\
\hline
\end{tabular}
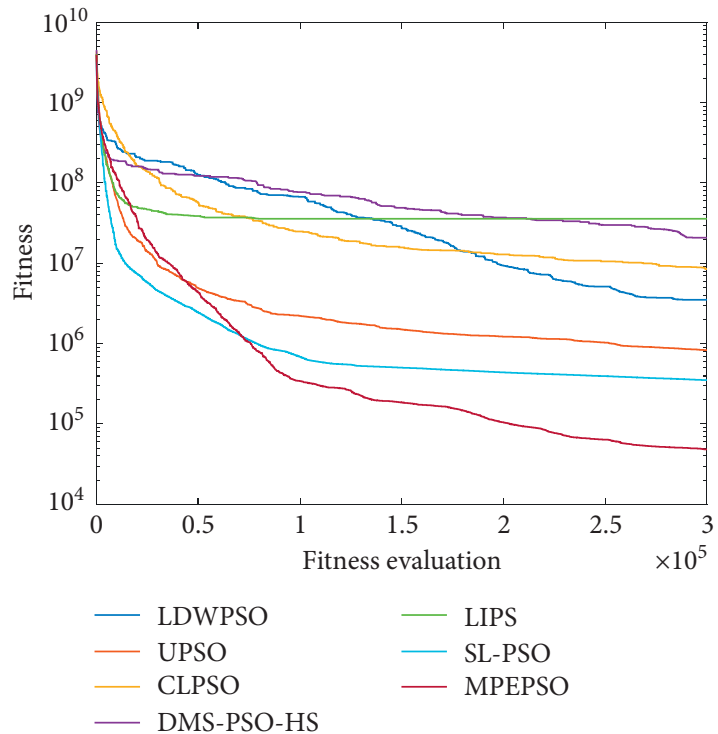

(a)

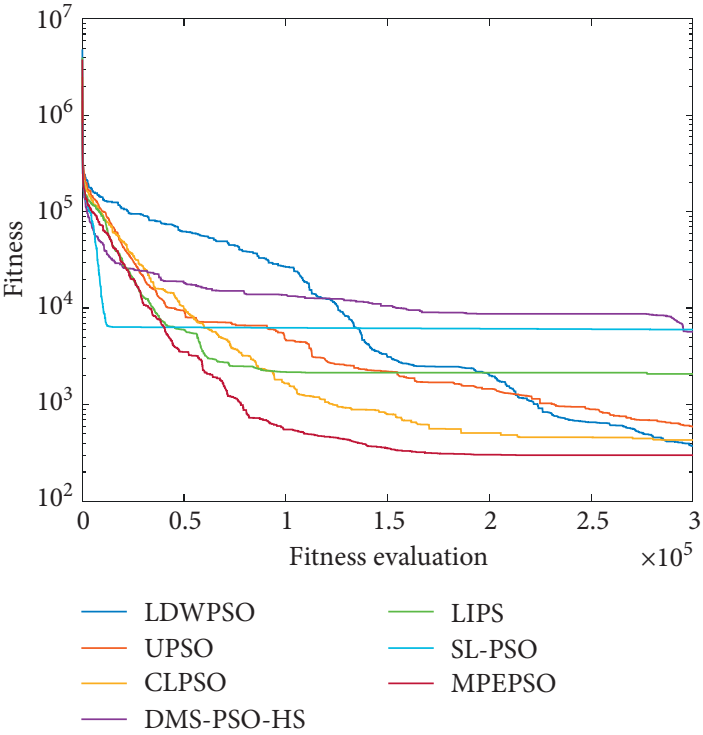

(b)

Figure 3: Continued. 

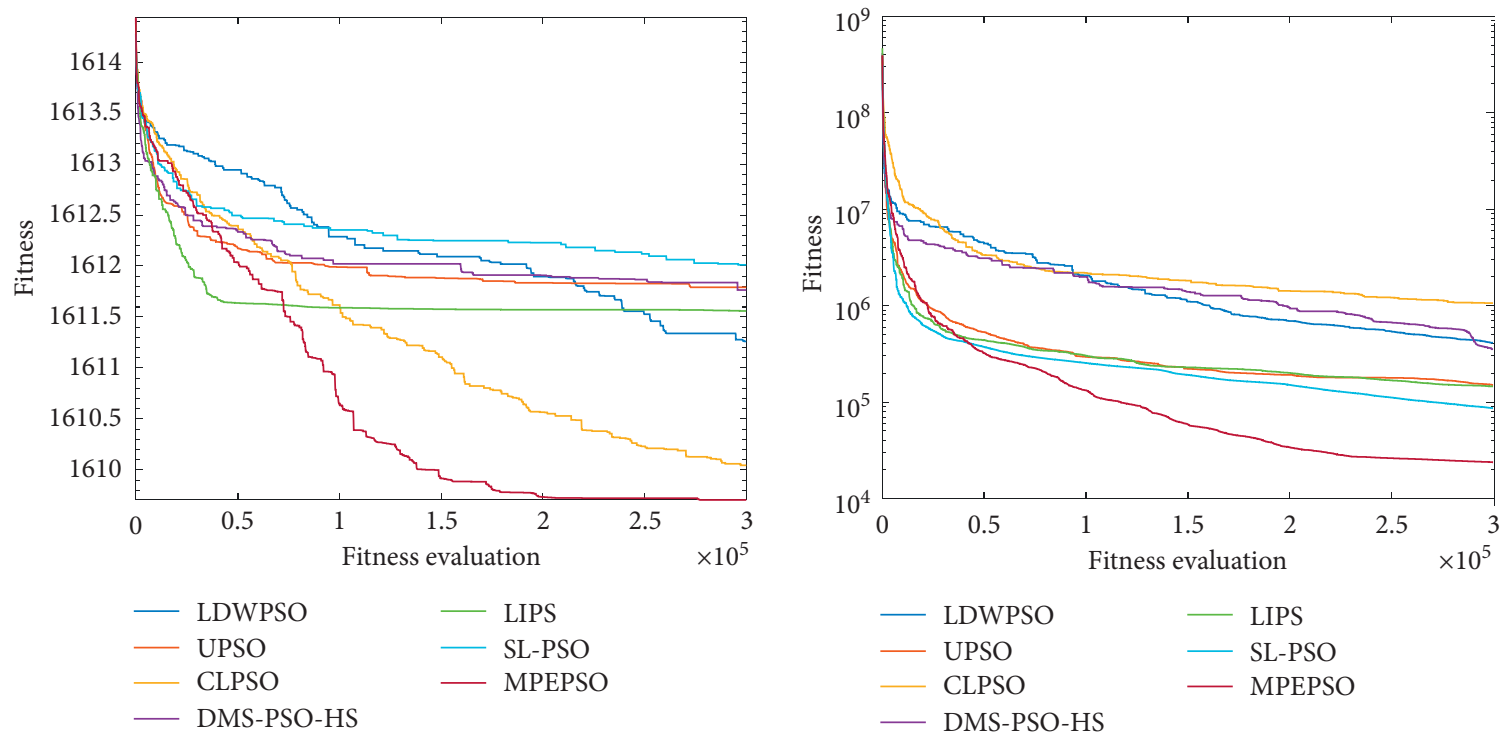

(c)
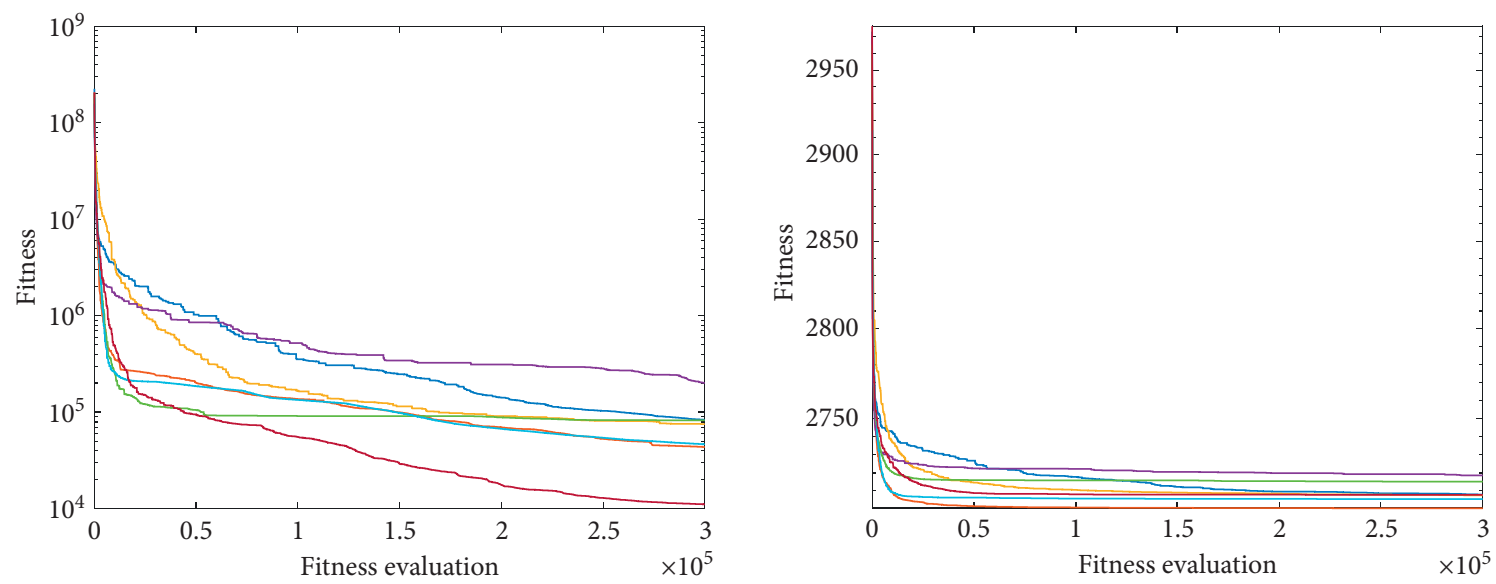

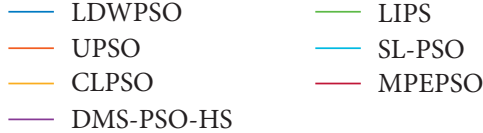

(e) (d)

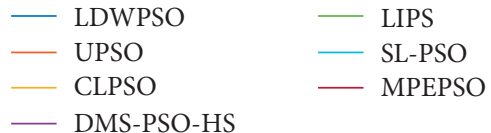

(f)

Figure 3: Median convergence graphs of six functions on 30-dimensional CEC2014 test suite: (a) F1, (b) F3, (c) F16, (d) F17, (e) F21, and (f) F25.

Table 16: Parameter settings.

\begin{tabular}{lc}
\hline Algorithm & Parameter settings \\
\hline CS [46] & $N=15, p_{a}=0.25, \lambda=1.5$ \\
FA [47] & $N=50, \alpha=0.5, \gamma=1, \beta_{0}=1, \beta_{\min }=0.2$ \\
SCA [48] & $N=30, a=2$ \\
GCMBO [49] & $N=50, S_{\max }=1.0, \mathrm{BAR}=5 / 12$, peri $=1.2, p=5 / 12$ \\
L-SHADE [50] & $N=18 D \sim 4, r^{\text {arc }}=2.6, p=0.11, H=6$ \\
\hline
\end{tabular}

and CLPSO have higher computational costs compared with LDWPSO and MPEPSO. Because MPEPSO adaptively uses the searching strategies in LDWPSO, UPSO, and CLPSO, it is reasonable that MPEPSO ranks in the middle of the component algorithms. The results show that MPEPSO has a competitive performance on the tests of computational time. 
TABLE 17: Computational results on 10-dimensional problems.

\begin{tabular}{|c|c|c|c|c|c|c|c|}
\hline & & CS & FA & SCA & GCMBO & L-SHADE & MPEPSO \\
\hline \multirow{3}{*}{$\mathrm{F} 1$} & Rank & 2 & 4 & 5 & 6 & 1 & 3 \\
\hline & Mean & $2.59 E-06$ & $2.91 E+05$ & $5.76 E+06$ & $1.16 E+07$ & 0 & $1.04 E+03$ \\
\hline & Std & $5.31 E-06$ & $2.73 E+05$ & $2.25 E+06$ & $1.46 E+07$ & 0 & $9.65 E+02$ \\
\hline \multirow{3}{*}{$\mathrm{F} 2$} & Rank & 2 & 4 & 5 & 6 & 1 & 3 \\
\hline & Mean & $4.07 E-13$ & $2.01 E+03$ & $5.04 E+08$ & $5.89 E+08$ & 0 & $1.04 E+03$ \\
\hline & Std & $2.23 E-12$ & $2.51 E+03$ & $1.77 E+08$ & $5.08 E+08$ & 0 & $1.18 E+03$ \\
\hline \multirow{3}{*}{ F3 } & Rank & 1 & 4 & 5 & 6 & 1 & 3 \\
\hline & Mean & 0 & $1.70 E+03$ & $3.19 E+03$ & $1.33 E+04$ & 0 & $2.53 E+01$ \\
\hline & Std & 0 & $1.77 E+03$ & $1.86 E+03$ & $1.01 E+04$ & 0 & $5.06 E+01$ \\
\hline \multirow{3}{*}{$\mathrm{F} 4$} & Rank & 1 & 4 & 5 & 6 & 3 & 2 \\
\hline & Mean & $4.70 E+00$ & $3.28 E+01$ & $5.45 E+01$ & $9.70 E+01$ & $3.14 E+01$ & $2.39 E+01$ \\
\hline & Std & $1.18 E+01$ & $7.72 E+00$ & $1.97 E+01$ & $5.02 E+01$ & $1.02 E+01$ & $1.57 E+01$ \\
\hline \multirow{3}{*}{ F5 } & Rank & 5 & 3 & 6 & 4 & 1 & 2 \\
\hline & Mean & $2.01 E+01$ & $1.93 E+01$ & $2.04 E+01$ & $2.01 E+01$ & $1.51 E+01$ & $1.62 E+01$ \\
\hline & Std & $4.53 E-02$ & $3.65 E+00$ & $7.27 E-02$ & $7.22 E-02$ & $8.26 E+00$ & $8.23 E+00$ \\
\hline \multirow{3}{*}{ F6 } & Rank & 4 & 3 & 5 & 6 & 1 & 2 \\
\hline & Mean & $2.04 E+00$ & $5.81 E-02$ & $6.26 E+00$ & $6.74 E+00$ & 0 & $1.19 E-02$ \\
\hline & Std & $1.20 E+00$ & $1.94 E-02$ & $9.30 E-01$ & $1.56 E+00$ & 0 & $4.92 E-02$ \\
\hline \multirow{3}{*}{ F7 } & Rank & 4 & 3 & 6 & 5 & 1 & 2 \\
\hline & Mean & $4.40 E-02$ & $3.12 E-02$ & $8.59 E+00$ & $3.64 E+00$ & $2.30 E-03$ & $3.08 E-02$ \\
\hline & Std & $1.54 E-02$ & $2.41 E-02$ & $2.45 E+00$ & $1.45 E+00$ & $6.87 E-03$ & $2.14 E-02$ \\
\hline \multirow{3}{*}{ F8 } & Rank & 2 & 4 & 6 & 5 & 1 & 3 \\
\hline & Mean & $1.93 E-05$ & $6.33 E+00$ & $3.62 E+01$ & $1.50 E+01$ & 0 & $9.62 E-01$ \\
\hline & Std & $1.06 E-04$ & $2.75 E+00$ & $5.06 E+00$ & $4.86 E+00$ & 0 & $1.03 E+00$ \\
\hline \multirow{3}{*}{ F9 } & Rank & 4 & 3 & 6 & 5 & 1 & 2 \\
\hline & Mean & $1.26 E+01$ & $6.07 E+00$ & $3.96 E+01$ & $3.49 E+01$ & $2.29 E+00$ & $4.78 E+00$ \\
\hline & Std & $4.91 E+00$ & $3.49 E+00$ & $6.95 E+00$ & $1.07 E+01$ & $9.83 E-01$ & $1.89 E+00$ \\
\hline \multirow{3}{*}{ F10 } & Rank & 2 & 5 & 6 & 4 & 1 & 3 \\
\hline & Mean & $1.91 E+01$ & $2.71 E+02$ & $8.75 E+02$ & $2.08 E+02$ & $1.46 E-02$ & $4.22 E+01$ \\
\hline & Std & $1.07 E+01$ & $2.13 E+02$ & $2.02 E+02$ & $1.32 E+02$ & $2.69 E-02$ & $5.53 E+01$ \\
\hline \multirow{3}{*}{ F11 } & Rank & 4 & 3 & 6 & 5 & 1 & 2 \\
\hline & Mean & $5.51 E+02$ & $2.23 E+02$ & $1.20 E+03$ & $9.86 E+02$ & $3.26 E+01$ & $1.08 E+02$ \\
\hline & Std & $1.63 E+02$ & $2.36 E+02$ & $1.65 E+02$ & $3.01 E+02$ & $4.64 E+01$ & $1.04 E+02$ \\
\hline \multirow{3}{*}{ F12 } & Rank & 3 & 1 & 6 & 5 & 2 & 4 \\
\hline & Mean & $1.90 E-01$ & $1.56 E-02$ & $1.09 E+00$ & $5.99 E-01$ & $6.74 E-02$ & $2.05 E-01$ \\
\hline & Std & $4.48 E-02$ & $1.48 E-02$ & $1.95 E-01$ & $2.39 E-01$ & $1.78 E-02$ & $1.71 E-01$ \\
\hline \multirow{3}{*}{ F13 } & Rank & 4 & 2 & 6 & 5 & 1 & 3 \\
\hline & Mean & $2.16 E-01$ & $6.16 E-02$ & $5.37 E-01$ & $4.21 E-01$ & $4.64 E-02$ & $7.19 E-02$ \\
\hline & Std & $5.71 E-02$ & $2.09 E-02$ & $1.02 E-01$ & $1.59 E-01$ & $1.54 E-02$ & $3.33 E-02$ \\
\hline \multirow{3}{*}{ F14 } & Rank & 3 & 4 & 5 & 6 & 1 & 2 \\
\hline & Mean & $1.86 E-01$ & $2.40 E-01$ & $8.41 E-01$ & $3.02 E+00$ & $7.53 E-02$ & $8.78 E-02$ \\
\hline & Std & $4.44 E-02$ & $7.00 E-02$ & $3.48 E-01$ & $2.26 E+00$ & $2.73 E-02$ & $3.16 E-02$ \\
\hline \multirow{3}{*}{ F15 } & Rank & 4 & 3 & 5 & 6 & 1 & 2 \\
\hline & Mean & $1.14 E+00$ & $8.07 E-01$ & $6.99 E+00$ & $7.34 E+00$ & $3.73 E-01$ & $6.79 E-01$ \\
\hline & Std & $3.05 E-01$ & $2.91 E-01$ & $1.53 E+00$ & $4.14 E+00$ & $5.91 E-02$ & $1.60 E-01$ \\
\hline \multirow{3}{*}{ F16 } & Rank & 4 & 3 & 5 & 6 & 2 & 1 \\
\hline & Mean & $2.91 E+00$ & $2.78 E+00$ & $3.21 E+00$ & $3.33 E+00$ & $1.25 E+00$ & $1.20 E+00$ \\
\hline & Std & $2.17 E-01$ & $4.26 E-01$ & $2.62 E-01$ & $3.16 E-01$ & $3.30 E-01$ & $5.78 E-01$ \\
\hline \multirow{3}{*}{ F17 } & Rank & 2 & 4 & 5 & 6 & 1 & 3 \\
\hline & Mean & $2.59 E+01$ & $2.14 E+03$ & $1.62 E+04$ & $1.36 E+06$ & $1.05 E+00$ & $8.92 E+02$ \\
\hline & Std & $2.85 E+01$ & $1.50 E+03$ & $1.33 E+04$ & $1.70 E+06$ & $1.15 E+00$ & $9.33 E+02$ \\
\hline \multirow{3}{*}{ F18 } & Rank & 2 & 4 & 5 & 6 & 1 & 3 \\
\hline & Mean & $1.20 E+00$ & $7.22 E+03$ & $1.48 E+04$ & $3.71 E+06$ & $2.28 E-01$ & $9.82 E+02$ \\
\hline & Std & $5.77 E-01$ & $7.60 E+03$ & $1.53 E+04$ & $1.11 E+07$ & $1.94 E-01$ & $1.06 E+03$ \\
\hline
\end{tabular}


Table 17: Continued.

\begin{tabular}{|c|c|c|c|c|c|c|c|}
\hline & & $\mathrm{CS}$ & FA & SCA & GCMBO & L-SHADE & MPEPSO \\
\hline \multirow{3}{*}{ F19 } & Rank & 3 & 4 & 5 & 6 & 1 & 2 \\
\hline & Mean & $1.15 E+00$ & $1.99 E+00$ & $4.68 E+00$ & $5.87 E+00$ & $7.97 E-02$ & $2.77 E-01$ \\
\hline & Std & $2.86 E-01$ & $5.99 E-01$ & $6.54 E-01$ & $4.38 E+00$ & $4.43 E-02$ & $3.83 E-01$ \\
\hline \multirow{3}{*}{ F20 } & Rank & 2 & 4 & 5 & 6 & 1 & 3 \\
\hline & Mean & $9.04 E-01$ & $7.77 E+02$ & $1.21 E+03$ & $6.53 E+03$ & $1.42 E-01$ & $5.27 E+01$ \\
\hline & Std & $5.00 E-01$ & $1.33 E+03$ & $1.67 E+03$ & $9.36 E+03$ & $1.17 E-01$ & $6.38 E+01$ \\
\hline \multirow{3}{*}{ F 21} & Rank & 2 & 4 & 5 & 6 & 1 & 3 \\
\hline & Mean & $9.91 E-01$ & $2.57 E+03$ & $5.72 E+03$ & $9.06 E+05$ & $4.11 E-01$ & $3.30 E+01$ \\
\hline & Std & $6.30 E-01$ & $2.82 E+03$ & $3.67 E+03$ & $8.61 E+05$ & $2.99 E-01$ & $4.70 E+01$ \\
\hline \multirow{3}{*}{$\mathrm{F} 22$} & Rank & 3 & 4 & 5 & 6 & 1 & 2 \\
\hline & Mean & $8.83 E+00$ & $2.99 E+01$ & $5.67 E+01$ & $7.20 E+01$ & $8.18 E-02$ & $7.32 E+00$ \\
\hline & Std & $7.33 E+00$ & $9.16 E+00$ & $2.38 E+01$ & $7.88 E+01$ & $3.06 E-02$ & $9.52 E+00$ \\
\hline \multirow{3}{*}{ F23 } & Rank & 2 & 1 & 6 & 5 & 2 & 2 \\
\hline & Mean & $3.29 E+02$ & $3.18 E+02$ & $3.39 E+02$ & $3.34 E+02$ & $3.29 E+02$ & $3.29 E+02$ \\
\hline & Std & $2.89 E-13$ & $6.01 E+01$ & $3.15 E+00$ & $3.84 E+01$ & $2.89 E-13$ & $2.89 E-13$ \\
\hline \multirow{3}{*}{ F 24} & Rank & 4 & 3 & 5 & 6 & 1 & 2 \\
\hline & Mean & $1.20 E+02$ & $1.13 E+02$ & $1.49 E+02$ & $1.54 E+02$ & $1.08 E+02$ & $1.11 E+02$ \\
\hline & Std & $5.92 E+00$ & $4.05 E+00$ & $8.00 E+00$ & $1.63 E+01$ & $1.25 E+00$ & $2.43 E+00$ \\
\hline \multirow{3}{*}{ F25 } & Rank & 3 & 4 & 5 & 6 & 2 & 1 \\
\hline & Mean & $1.48 E+02$ & $1.68 E+02$ & $1.92 E+02$ & $2.00 E+02$ & $1.40 E+02$ & $1.35 E+02$ \\
\hline & Std & $2.34 E+01$ & $4.05 E+01$ & $1.86 E+01$ & $1.23 E+01$ & $4.35 E+01$ & $2.79 E+01$ \\
\hline \multirow{3}{*}{ F26 } & Rank & 4 & 3 & 6 & 5 & 1 & 2 \\
\hline & Mean & $1.00 E+02$ & $1.00 E+02$ & $1.01 E+02$ & $1.01 E+02$ & $1.00 E+02$ & $1.00 E+02$ \\
\hline & Std & $5.56 E-02$ & $2.50 E-02$ & $1.45 E-01$ & $2.27 E-01$ & $1.55 E-02$ & $2.18 E-02$ \\
\hline \multirow{3}{*}{$\mathrm{F} 27$} & Rank & 4 & 3 & 5 & 6 & 1 & 2 \\
\hline & Mean & $2.15 E+02$ & $1.32 E+02$ & $2.21 E+02$ & $3.52 E+02$ & $4.78 E+01$ & $5.16 E+01$ \\
\hline & Std & $1.80 E+02$ & $1.51 E+02$ & $1.98 E+02$ & $1.73 E+02$ & $1.22 E+02$ & $1.31 E+02$ \\
\hline \multirow{3}{*}{$\mathrm{F} 28$} & Rank & 1 & 3 & 5 & 6 & 2 & 4 \\
\hline & Mean & $3.76 E+02$ & $3.99 E+02$ & $4.61 E+02$ & $5.19 E+02$ & $3.80 E+02$ & $4.22 E+02$ \\
\hline & Std & $5.12 E+00$ & $5.03 E+01$ & $4.76 E+01$ & $1.22 E+02$ & $3.11 E+01$ & $5.35 E+01$ \\
\hline \multirow{3}{*}{ F29 } & Rank & 1 & 4 & 5 & 6 & 2 & 3 \\
\hline & Mean & $2.15 E+02$ & $6.00 E+02$ & $2.46 E+03$ & $6.32 E+04$ & $2.22 E+02$ & $2.87 E+02$ \\
\hline & Std & $1.91 E+01$ & $1.17 E+02$ & $1.95 E+03$ & $3.14 E+05$ & $5.08 E-01$ & $3.62 E+01$ \\
\hline \multirow{3}{*}{ F30 } & Rank & 2 & 3 & 5 & 6 & 1 & 4 \\
\hline & Mean & $5.09 E+02$ & $7.17 E+02$ & $1.25 E+03$ & $2.26 E+03$ & $4.64 E+02$ & $7.33 E+02$ \\
\hline & Std & $5.96 E+01$ & $1.90 E+02$ & $3.32 E+02$ & $1.91 E+03$ & $6.79 E+00$ & $1.86 E+02$ \\
\hline
\end{tabular}

4.5. Application to Engineering Design Problems. In this section, MPEPSO is applied to solve four real-world engineering design problems including the tension/compression spring design problem, the pressure vessel design problem, the cantilever beam design problem, and the gear train design problem. To solve these four constrained problems, the penalty function method is used to handle the constraints. The idea of the penalty function method is to incorporate constraints into the objective function, and the constrained problems can be transformed into an unconstrained problem. When a certain constraint is violated, we multiply the constraint by a penalty value and added the result to the objective function. In this paper, the penalty is set at a large value, $10 E+10$, for these four problems.

The performance of MPEPSO is compared with that of two recently proposed novel metaheuristic algorithms and one recently proposed PSO variant. These algorithms are listed as follows: (i) SCA: sine cosine algorithm [48]

(ii) WOA: whale optimization algorithm [56]

(iii) BLPSO: biogeography-based learning particle swarm optimization [27]

SCA is a population-based optimization algorithm that has received considerable attention. SCA uses a mathematical model based on sine and cosine functions to reach better solutions [48]. WOA is a nature-inspired optimization algorithm that has been studied by many researchers. WOA is inspired by the behavior of humpback whales [56]. BLPSO is a recently proposed PSO variant that introduced the migration operator of the biogeography-based optimization (BBO) algorithm [57] into CLPSO.

To conduct a fair competition, the parameters for the comparison algorithms are the same as the original codes of the papers $[27,48,56]$. MPEPSO keeps the same parameter setting. The parameter settings for each algorithm are listed in Table 25. 
TABLE 18: Wilcoxon signed-rank test for 30 runs on 10-dimensional problems.

\begin{tabular}{|c|c|c|c|c|c|}
\hline & $\mathrm{CS}$ & FA & SCA & GCMBO & L-SHADE \\
\hline F1 & - & + & + & + & - \\
\hline $\mathrm{F} 2$ & - & $=$ & + & + & - \\
\hline F3 & - & + & + & + & - \\
\hline $\mathrm{F} 4$ & - & + & + & + & $=$ \\
\hline F5 & $=$ & $=$ & + & $=$ & $=$ \\
\hline F6 & + & + & + & + & - \\
\hline F7 & + & $=$ & + & + & - \\
\hline F8 & - & + & + & + & - \\
\hline F9 & + & $=$ & + & + & - \\
\hline F10 & $=$ & + & + & + & - \\
\hline F11 & + & + & + & + & - \\
\hline F12 & $=$ & - & + & + & - \\
\hline F13 & + & $=$ & + & + & - \\
\hline F14 & + & + & + & + & $=$ \\
\hline F15 & + & $=$ & + & + & - \\
\hline F16 & + & + & + & + & $=$ \\
\hline F17 & - & + & + & + & - \\
\hline F18 & - & + & + & + & - \\
\hline F19 & + & + & + & + & - \\
\hline F20 & - & + & + & + & - \\
\hline $\mathrm{F} 21$ & - & + & + & + & - \\
\hline F22 & $=$ & + & + & + & - \\
\hline F23 & $=$ & - & + & + & $=$ \\
\hline F24 & + & + & + & + & - \\
\hline F25 & + & + & + & + & $=$ \\
\hline F26 & + & $=$ & + & + & $=$ \\
\hline F27 & + & + & + & + & $=$ \\
\hline F28 & - & $=$ & + & + & - \\
\hline F29 & - & + & + & + & - \\
\hline F30 & - & $=$ & + & + & - \\
\hline$+1=1-$ & $13 / 5 / 12$ & $19 / 9 / 2$ & $30 / 0 / 0$ & $29 / 1 / 0$ & $0 / 8 / 22$ \\
\hline
\end{tabular}

TABLE 19: The rank of the six algorithms on 10-dimensional problems.

\begin{tabular}{|c|c|c|c|c|c|c|}
\hline Rank & $\mathrm{CS}$ & FA & SCA & GCMBO & L-SHADE & MPEPSO \\
\hline Total rank & 84 & 101 & 160 & 168 & 38 & 75 \\
\hline Ave. rank & 2.8 & 3.366667 & 5.333333 & 5.6 & 1.266667 & 2.5 \\
\hline Final rank & 6 & 3 & 4 & 5 & 1 & 2 \\
\hline
\end{tabular}

In SCA, constant $a$ is used to control the range of sine and cosine. In WOA, $\vec{r}$ is a random vector in $[0,1]$ and parameter $\vec{a}$ is linearly decreased from 2 to 0 to control the coefficient vector $\vec{A}$. In BLPSO, $I$ is the maximum immigration rate and $E$ is the maximum emigration rate.

The maximum number of function evaluations is set to $10,000 \mathrm{D}$. For each problem, all algorithms run 30 times independently.

4.5.1. Tension/Compression Spring Design Problem. Coil springs are used in various real-world applications. The purpose of the spring design problem is to design a minimum-mass spring [58]. As shown in Figure 4 [59], for a given applied load $P$, three design variables, wire diameter $d\left(x_{1}\right)$, mean coil diameter $D\left(x_{2}\right)$, number of active coils $N\left(x_{3}\right)$, need to be optimized to design a minimum-mass spring.
This is a nonlinear programming problem and the final formulation for the spring design problem is shown as follows [60]:

$$
\begin{aligned}
& \min f(x)=\left(x_{3}+2\right) x_{2} x_{1}^{2}, \\
& \text { s.t. } g_{1}(x)=1-\frac{x_{2}^{3} x_{3}}{71,785 x_{1}^{4}} \leq 0, \\
& g_{2}(x)=\frac{4 x_{2}^{2}-x_{1} x_{2}}{12,566\left(x_{2} x_{1}^{3}-x_{1}^{4}\right)}+\frac{1}{5,108 x_{1}^{2}}-1 \leq 0, \\
& g_{3}(x)=1-\frac{140.45 x_{1}}{x_{2}^{2} x_{3}} \leq 0, \\
& g_{4}(x)=\frac{x_{1}+x_{2}}{1.5}-1 \leq 0,
\end{aligned}
$$


TABle 20: Computational results on 30-dimensional problems.

\begin{tabular}{|c|c|c|c|c|c|c|c|}
\hline & & CS & FA & SCA & GCMBO & L-SHADE & MPEPSO \\
\hline \multirow{3}{*}{$\mathrm{F} 1$} & Rank & 3 & 4 & 6 & 5 & 1 & 2 \\
\hline & Mean & $7.37 E+05$ & $2.19 E+06$ & $2.40 E+08$ & $2.33 E+08$ & $8.53 E-15$ & $6.33 E+04$ \\
\hline & Std & $4.66 E+05$ & $1.01 E+06$ & $7.92 E+07$ & $2.15 E+08$ & $7.08 E-15$ & $4.66 E+04$ \\
\hline \multirow{3}{*}{ F2 } & Rank & 5 & 3 & 6 & 4 & 1 & 2 \\
\hline & Mean & $1.00 E+10$ & $7.83 E+03$ & $1.64 E+10$ & $7.01 E+09$ & 0 & $2.16 E-05$ \\
\hline & Std & $0.00 E+00$ & $8.57 E+03$ & $2.72 E+09$ & $3.57 E+09$ & 0 & $4.62 E-05$ \\
\hline \multirow{3}{*}{ F3 } & Rank & 2 & 4 & 5 & 6 & 1 & 3 \\
\hline & Mean & $1.15 E-11$ & $5.47 E+03$ & $3.73 E+04$ & $5.80 E+04$ & 0 & $3.66 E-02$ \\
\hline & Std & $5.18 E-11$ & $2.62 E+03$ & $5.12 E+03$ & $8.17 E+04$ & 0 & $8.17 E-02$ \\
\hline \multirow{3}{*}{$\mathrm{F} 4$} & Rank & 3 & 4 & 5 & 6 & 1 & 2 \\
\hline & Mean & $2.21 E+01$ & $8.02 E+01$ & $9.72 E+02$ & $1.70 E+03$ & $5.12 E-14$ & $2.47 E+00$ \\
\hline & Std & $3.20 E+01$ & $1.10 E+01$ & $2.30 E+02$ & $8.66 E+02$ & $2.73 E-14$ & $1.18 E+01$ \\
\hline \multirow{3}{*}{ F5 } & Rank & 5 & 1 & 6 & 3 & 2 & 4 \\
\hline & Mean & $2.08 E+01$ & $2.00 E+01$ & $2.09 E+01$ & $2.04 E+01$ & $2.01 E+01$ & $2.05 E+01$ \\
\hline & Std & $6.58 E-02$ & $4.09 E-04$ & $5.67 E-02$ & $9.13 E-02$ & $4.75 E-02$ & $4.55 E-01$ \\
\hline \multirow{3}{*}{ F6 } & Rank & 4 & 2 & 6 & 5 & 1 & 3 \\
\hline & Mean & $2.40 E+01$ & $6.28 E-01$ & $3.37 E+01$ & $2.82 E+01$ & 0 & $5.54 E+00$ \\
\hline & Std & $2.42 E+00$ & $5.09 E-01$ & $2.47 E+00$ & $2.51 E+00$ & 0 & $2.30 E+00$ \\
\hline \multirow{3}{*}{ F7 } & Rank & 3 & 2 & 6 & 5 & 1 & 4 \\
\hline & Mean & $5.33 E-03$ & $3.19 E-03$ & $1.34 E+02$ & $3.82 E+01$ & 0 & $9.68 E-03$ \\
\hline & Std & $1.04 E-02$ & $2.46 E-03$ & $3.10 E+01$ & $1.96 E+01$ & 0 & $1.13 E-02$ \\
\hline \multirow{3}{*}{ F8 } & Rank & 2 & 4 & 6 & 5 & 1 & 3 \\
\hline & Mean & $1.30 E+01$ & $2.87 E+01$ & $2.37 E+02$ & $7.17 E+01$ & $1.14 E-13$ & $2.01 E+01$ \\
\hline & Std & $5.57 E+00$ & $9.30 E+00$ & $1.89 E+01$ & $1.99 E+01$ & 0 & $7.46 E+00$ \\
\hline \multirow{3}{*}{ F9 } & Rank & 4 & 2 & 6 & 5 & 1 & 3 \\
\hline & Mean & $1.41 E+02$ & $2.73 E+01$ & $2.66 E+02$ & $1.97 E+02$ & $6.60 E+00$ & $3.87 E+01$ \\
\hline & Std & $3.34 E+01$ & $8.72 E+00$ & $2.04 E+01$ & $4.53 E+01$ & $1.34 E+00$ & $1.01 E+01$ \\
\hline \multirow{3}{*}{ F10 } & Rank & 2 & 4 & 6 & 5 & 1 & 3 \\
\hline & Mean & $2.11 E+02$ & $1.31 E+03$ & $5.98 E+03$ & $1.42 E+03$ & $5.55 E-03$ & $4.63 E+02$ \\
\hline & Std & $1.35 E+02$ & $4.82 E+02$ & $5.15 E+02$ & $5.25 E+02$ & $1.08 E-02$ & $1.86 E+02$ \\
\hline \multirow{3}{*}{ F11 } & Rank & 4 & 2 & 6 & 5 & 1 & 3 \\
\hline & Mean & $3.63 E+03$ & $1.63 E+03$ & $6.91 E+03$ & $4.78 E+03$ & $1.25 E+03$ & $2.49 E+03$ \\
\hline & Std & $2.64 E+02$ & $6.33 E+02$ & $4.27 E+02$ & $5.55 E+02$ & $1.56 E+02$ & $4.47 E+02$ \\
\hline \multirow{3}{*}{ F12 } & Rank & 5 & 1 & 6 & 4 & 2 & 3 \\
\hline & Mean & $6.63 E-01$ & $9.91 E-03$ & $2.48 E+00$ & $6.29 E-01$ & $1.54 E-01$ & $5.01 E-01$ \\
\hline & Std & $1.51 E-01$ & $3.71 E-03$ & $2.90 E-01$ & $1.93 E-01$ & $2.25 E-02$ & $6.08 E-01$ \\
\hline \multirow{3}{*}{ F13 } & Rank & 4 & 2 & 6 & 5 & 1 & 3 \\
\hline & Mean & $3.87 E-01$ & $1.58 E-01$ & $2.85 E+00$ & $7.38 E-01$ & $1.22 E-01$ & $1.72 E-01$ \\
\hline & Std & $7.55 E-02$ & $3.84 E-02$ & $3.99 E-01$ & $4.53 E-01$ & $1.39 E-02$ & $4.65 E-02$ \\
\hline \multirow{3}{*}{ F14 } & Rank & 4 & 3 & 6 & 5 & 2 & 1 \\
\hline & Mean & $2.91 E-01$ & $2.75 E-01$ & $4.65 E+01$ & $1.74 E+01$ & $2.40 E-01$ & $2.27 E-01$ \\
\hline & Std & $1.14 E-01$ & $4.06 E-02$ & $1.03 E+01$ & $6.03 E+00$ & $2.68 E-02$ & $4.38 E-02$ \\
\hline \multirow{3}{*}{ F15 } & Rank & 4 & 2 & 6 & 5 & 1 & 3 \\
\hline & Mean & $2.04 E+01$ & $3.39 E+00$ & $2.21 E+03$ & $2.10 E+03$ & $2.15 E+00$ & $3.75 E+00$ \\
\hline & Std & $5.67 E+00$ & $5.82 E-01$ & $1.69 E+03$ & $7.87 E+03$ & $2.16 E-01$ & $1.03 E+00$ \\
\hline \multirow{3}{*}{ F16 } & Rank & 5 & 3 & 6 & 4 & 1 & 2 \\
\hline & Mean & $1.23 E+01$ & $1.16 E+01$ & $1.26 E+01$ & $1.19 E+01$ & $8.55 E+00$ & $9.65 E+00$ \\
\hline & Std & $2.28 E-01$ & $5.29 E-01$ & $2.66 E-01$ & $6.52 E-01$ & $3.60 E-01$ & $7.22 E-01$ \\
\hline \multirow{3}{*}{ F17 } & Rank & 2 & 4 & 5 & 6 & 1 & 3 \\
\hline & Mean & $2.43 E+03$ & $1.42 E+05$ & $6.19 E+06$ & $3.20 E+07$ & $2.39 E+02$ & $2.35 E+04$ \\
\hline & Std & $1.22 E+03$ & $6.72 E+04$ & $3.15 E+06$ & $2.10 E+07$ & $1.04 E+02$ & $1.72 E+04$ \\
\hline \multirow{3}{*}{ F18 } & Rank & 2 & 4 & 5 & 6 & 1 & 3 \\
\hline & Mean & $8.92 E+01$ & $3.13 E+03$ & $1.61 E+08$ & $2.45 E+08$ & $6.31 E+00$ & $1.44 E+03$ \\
\hline & Std & $3.71 E+01$ & $2.87 E+03$ & $8.48 E+07$ & $2.30 E+08$ & $2.55 E+00$ & $2.24 E+03$ \\
\hline
\end{tabular}


TABle 20: Continued.

\begin{tabular}{|c|c|c|c|c|c|c|c|}
\hline & & CS & FA & SCA & GCMBO & L-SHADE & MPEPSO \\
\hline \multirow{3}{*}{ F19 } & Rank & 3 & 4 & 5 & 6 & 1 & 2 \\
\hline & Mean & $8.37 E+00$ & $9.01 E+00$ & $8.62 E+01$ & $9.31 E+01$ & $3.74 E+00$ & $4.29 E+00$ \\
\hline & Std & $1.59 E+00$ & $1.83 E+00$ & $1.96 E+01$ & $4.06 E+01$ & $6.75 E-01$ & $1.24 E+00$ \\
\hline \multirow{3}{*}{ F20 } & Rank & 2 & 4 & 5 & 6 & 1 & 3 \\
\hline & Mean & $1.25 E+02$ & $4.33 E+02$ & $1.24 E+04$ & $4.84 E+04$ & $2.72 E+00$ & $1.99 E+02$ \\
\hline & Std & $6.26 E+01$ & $3.24 E+02$ & $5.39 E+03$ & $2.69 E+04$ & $1.03 E+00$ & $1.20 E+02$ \\
\hline \multirow{3}{*}{ F21 } & Rank & 2 & 4 & 5 & 6 & 1 & 3 \\
\hline & Mean & $9.13 E+02$ & $7.14 E+04$ & $1.34 E+06$ & $7.64 E+06$ & $1.09 E+02$ & $1.08 E+04$ \\
\hline & Std & $2.98 E+02$ & $5.38 E+04$ & $7.38 E+05$ & $1.27 E+07$ & $7.95 E+01$ & $7.04 E+03$ \\
\hline \multirow{3}{*}{ F22 } & Rank & 4 & 3 & 5 & 6 & 1 & 2 \\
\hline & Mean & $2.83 E+02$ & $2.22 E+02$ & $7.58 E+02$ & $8.66 E+02$ & $2.37 E+01$ & $1.46 E+02$ \\
\hline & Std & $1.29 E+02$ & $9.30 E+01$ & $1.35 E+02$ & $2.55 E+02$ & $4.02 E+00$ & $5.08 E+01$ \\
\hline \multirow{3}{*}{ F23 } & Rank & 1 & 4 & 5 & 6 & 1 & 1 \\
\hline & Mean & $3.15 E+02$ & $3.15 E+02$ & $3.72 E+02$ & $3.78 E+02$ & $3.15 E+02$ & $3.15 E+02$ \\
\hline & Std & $1.73 E-13$ & $3.81 E-02$ & $1.67 E+01$ & $4.30 E+01$ & $5.78 E-14$ & $5.72 E-13$ \\
\hline \multirow{3}{*}{ F24 } & Rank & 5 & 2 & 1 & 6 & 3 & 4 \\
\hline & Mean & $2.30 E+02$ & $2.10 E+02$ & $2.02 E+02$ & $2.81 E+02$ & $2.25 E+02$ & $2.25 E+02$ \\
\hline & Std & $6.27 E+00$ & $1.17 E+01$ & $1.31 E+01$ & $1.47 E+01$ & $1.40 E+00$ & $2.65 E+00$ \\
\hline \multirow{3}{*}{$\mathrm{F} 25$} & Rank & 2 & 3 & 6 & 5 & 1 & 4 \\
\hline & Mean & $2.05 E+02$ & $2.05 E+02$ & $2.26 E+02$ & $2.21 E+02$ & $2.03 E+02$ & $2.08 E+02$ \\
\hline & Std & $2.17 E+00$ & $9.26 E-01$ & $7.44 E+00$ & $5.94 E+00$ & $4.25 E-02$ & $2.39 E+00$ \\
\hline \multirow{3}{*}{ F26 } & Rank & 4 & 2 & 3 & 5 & 1 & 6 \\
\hline & Mean & $1.04 E+02$ & $1.00 E+02$ & $1.02 E+02$ & $1.06 E+02$ & $1.00 E+02$ & $1.07 E+02$ \\
\hline & Std & $1.82 E+01$ & $4.53 E-02$ & $5.71 E-01$ & $1.88 E+01$ & $1.56 E-02$ & $2.53 E+01$ \\
\hline \multirow{3}{*}{$\mathrm{F} 27$} & Rank & 4 & 2 & 5 & 6 & 1 & 3 \\
\hline & Mean & $4.23 E+02$ & $3.28 E+02$ & $7.23 E+02$ & $9.47 E+02$ & 300 & $4.11 E+02$ \\
\hline & Std & $1.10 E+02$ & $3.61 E+01$ & $3.10 E+02$ & $2.66 E+02$ & $1.19 E-13$ & $4.93 E+01$ \\
\hline \multirow{3}{*}{ F28 } & Rank & 4 & 2 & 6 & 5 & 1 & 3 \\
\hline & Mean & $1.05 E+03$ & $8.33 E+02$ & $2.03 E+03$ & $1.33 E+03$ & $8.28 E+02$ & $9.26 E+02$ \\
\hline & Std & $1.41 E+02$ & $2.33 E+01$ & $3.12 E+02$ & $1.92 E+02$ & $2.44 E+01$ & $5.51 E+01$ \\
\hline \multirow{3}{*}{ F29 } & Rank & 3 & 4 & 6 & 5 & 1 & 2 \\
\hline & Mean & $1.08 E+03$ & $2.01 E+04$ & $1.30 E+07$ & $4.89 E+06$ & $7.17 E+02$ & $1.07 E+03$ \\
\hline & Std & $7.03 E+02$ & $2.86 E+03$ & $7.29 E+06$ & $3.45 E+06$ & $3.32 E+00$ & $2.56 E+02$ \\
\hline \multirow{3}{*}{ F30 } & Rank & 3 & 4 & 6 & 5 & 1 & 2 \\
\hline & Mean & $1.94 E+03$ & $5.30 E+03$ & $2.29 E+05$ & $1.31 E+05$ & $1.15 E+03$ & $1.85 E+03$ \\
\hline & Std & $9.28 E+02$ & $1.68 E+03$ & $1.05 E+05$ & $6.43 E+04$ & $4.19 E+02$ & $5.08 E+02$ \\
\hline
\end{tabular}

$0.05 \leq x_{1} \leq 2$

$0.25 \leq x_{2} \leq 1.3$

$$
2 \leq x_{3} \leq 15
$$

The objective function (14) is to minimize the mass of the spring. Constraints (15) (18) represent the deflection constraint, the shear stress constraint, the frequency constraint, and the outer diameter constraint, respectively. Finally, constraints (19) (21) represent the minimum and maximum size limits of three decision variables.

The best, mean, worst, and standard deviation values of the tension/compression spring design problem for the 30 runs are shown in Table 26. From this table, we can find that MPEPSO outperforms SCA, WOA, and BLPSO in terms of all comparison aspects.
The best solutions of MPEPSO, SCA, WOA, and BLPSO are shown in Table 27. As shown in this table, we can find that MPEPSO obtains the minimized mass of the spring comparing with other algorithms.

4.5.2. Pressure Vessel Design Problem. In this problem, a cylindrical pressure vessel is capped at both ends by hemispherical heads. The purpose of the pressure vessel design problem is to minimize the welding, the material, and forming costs [61]. As shown in Figure 5 [59], the design variables are the thicknesses of the shell $T_{s}\left(x_{1}\right)$, the thicknesses of the head $T_{h}\left(x_{2}\right)$, the inner radius of the shell $R\left(x_{3}\right)$, and the length of the shell $L\left(x_{4}\right)$.

In this design problem, $T_{s}$ and $T_{h}$ should be integer multiples of 0.0625 inch, while $R$ and $L$ are continuous. This is a mixed-integer nonlinear programming problem and the final formulation for the pressure vessel design problem is shown as follows [62]: 
TABLE 21: Wilcoxon signed-rank test for 30 runs on 30-dimensional problems.

\begin{tabular}{|c|c|c|c|c|c|}
\hline & CS & FA & SCA & GCMBO & L-SHADE \\
\hline $\mathrm{F} 1$ & + & + & + & + & - \\
\hline F2 & + & + & + & + & - \\
\hline F3 & - & + & + & + & - \\
\hline $\mathrm{F} 4$ & + & + & + & + & - \\
\hline F5 & $=$ & - & + & - & - \\
\hline F6 & + & - & + & + & - \\
\hline F7 & - & - & + & + & - \\
\hline F8 & - & + & + & + & - \\
\hline F9 & + & - & + & + & - \\
\hline F10 & - & + & + & + & - \\
\hline F11 & + & - & + & + & - \\
\hline F12 & + & - & + & + & - \\
\hline F13 & + & $=$ & + & + & - \\
\hline F14 & + & + & + & + & $=$ \\
\hline F15 & + & $=$ & + & + & - \\
\hline F16 & + & + & + & + & - \\
\hline F17 & - & + & + & + & - \\
\hline F18 & - & + & + & + & - \\
\hline F19 & + & + & + & + & $=$ \\
\hline F20 & - & + & + & + & - \\
\hline F21 & - & + & + & + & - \\
\hline F22 & + & + & + & + & - \\
\hline F23 & - & + & + & + & - \\
\hline F24 & + & - & - & + & $=$ \\
\hline F25 & - & - & + & + & - \\
\hline F26 & - & $=$ & - & - & - \\
\hline F27 & $=$ & - & + & + & - \\
\hline F28 & + & - & + & + & - \\
\hline F29 & + & + & + & + & - \\
\hline F30 & $=$ & + & + & + & - \\
\hline$+1=1-$ & $16 / 3 / 11$ & $17 / 3 / 10$ & $28 / 0 / 2$ & $28 / 0 / 2$ & $0 / 3 / 27$ \\
\hline
\end{tabular}

TABle 22: The rank of the six algorithms on 30-dimensional problems.

\begin{tabular}{lcccccc}
\hline Rank & CS & FA & SCA & GCMBO & L-SHADE & MPEPSO \\
\hline $\begin{array}{l}\text { Total } \\
\text { rank }\end{array}$ & 84 & 101 & 160 & 168 & 38 & 75 \\
$\begin{array}{l}\text { Ave. } \\
\text { rank }\end{array}$ & 2.8 & 3.366667 & 5.333333 & 5.6 & 1.266667 & 2.5 \\
$\begin{array}{l}\text { Final } \\
\text { rank }\end{array}$ & 6 & 3 & 4 & 5 & 1 & 2 \\
\hline
\end{tabular}

TABle 23: Computational time of the algorithms on 10 dimensions (time in seconds).

\begin{tabular}{lccccc}
\hline $10 D$ & $T_{0}$ & $T_{1}$ & $\widehat{T}_{2}$ & $\left(\widehat{T}_{2}-T_{1}\right) / T_{0}$ & Rank \\
\hline LDWPSO & 0.2958 & 0.2166 & 1.6306 & 4.7803 & 2 \\
UPSO & 0.2958 & 0.2166 & 1.9436 & 5.8385 & 5 \\
CLPSO & 0.2958 & 0.2166 & 1.911 & 5.7282 & 4 \\
DMS-PSO & 0.2958 & 0.2166 & 2.0728 & 6.2752 & 6 \\
LIPS & 0.2958 & 0.2166 & 8.0522 & 26.4895 & 7 \\
SL-PSO & 0.2958 & 0.2166 & 0.8052 & 1.9899 & 1 \\
MPEPSO & 0.2958 & 0.2166 & 1.8448 & 5.5044 & 3 \\
\hline
\end{tabular}

TABLE 24: Computational time of the algorithms on 30 dimensions (time in seconds).

\begin{tabular}{lccccc}
\hline $30 \mathrm{D}$ & $T_{0}$ & $T_{1}$ & $\widehat{T}_{2}$ & $\left(\widehat{T}_{2}-T_{1}\right) / T_{0}$ & Rank \\
\hline LDWPSO & 0.2958 & 0.3554 & 2.107 & 5.9216 & 2 \\
UPSO & 0.2958 & 0.3554 & 2.3694 & 6.8087 & 4 \\
CLPSO & 0.2958 & 0.3554 & 2.4946 & 7.2319 & 5 \\
DMS-PSO & 0.2958 & 0.3554 & 3.573 & 10.8776 & 6 \\
LIPS & 0.2958 & 0.3554 & 8.8836 & 28.831 & 7 \\
SL-PSO & 0.2958 & 0.3554 & 1.1682 & 2.7478 & 1 \\
MPEPSO & 0.2958 & 0.3554 & 2.2154 & 6.288 & 3 \\
\hline
\end{tabular}

TABle 25: Parameter settings for SCA, WOA, and BLPSO.

\begin{tabular}{lc}
\hline Algorithm & Parameter settings \\
\hline SCA & $N=30, a=2$ \\
WOA & $N=30, \vec{A}=2 \vec{a} \cdot \vec{r}-\vec{a}, \vec{C}=2 \cdot \vec{r}, \vec{a}=2 \sim 0$ \\
BLPSO & $N=40, \omega=0.9 \sim 0.2, c=1.49445, I=1, E=1$ \\
\hline
\end{tabular}

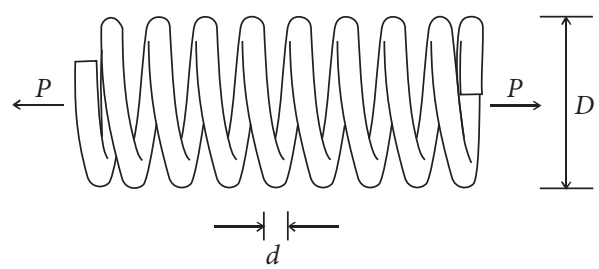

FIgURE 4: Tension/compression spring design problem.

TABLE 26: Computational results of the tension/compression spring design problem.

\begin{tabular}{lcccc}
\hline Algorithms & Best & Mean & Worst & Std \\
\hline MPEPSO & $\mathbf{0 . 0 1 2 6 6 7}$ & $\mathbf{0 . 0 1 2 8 2 9}$ & $\mathbf{0 . 0 1 3 1 1 7}$ & $\mathbf{0 . 0 0 0 1 3 6}$ \\
SCA & 0.012709 & 0.013033 & 0.014199 & 0.000264 \\
WOA & 0.012669 & 0.013649 & 0.017774 & 0.001178 \\
BLPSO & 0.012689 & 0.01309 & 0.013991 & 0.000343 \\
\hline
\end{tabular}

TABLE 27: Best results of the tension/compression spring design problem.

\begin{tabular}{|c|c|c|c|c|}
\hline \multirow{2}{*}{ Algorithms } & \multicolumn{3}{|c|}{ Optimal decision vector } & \multirow{2}{*}{ Optimum value } \\
\hline & $x_{1}$ & $x_{2}$ & $x_{3}$ & \\
\hline MPEPSO & 0.051872 & 0.361118 & 11.03631 & 0.012667 \\
\hline SCA & 0.051002 & 0.340188 & 12.36216 & 0.012709 \\
\hline WOA & 0.051233 & 0.34584 & 11.95642 & 0.012669 \\
\hline BLPSO & 0.052825 & 0.384655 & 9.821553 & 0.012689 \\
\hline
\end{tabular}

$$
\begin{aligned}
\min f(x)= & 0.6224 x_{1} x_{3} x_{4}+1.7781 x_{2} x_{3}^{2} \\
& +3.1661 x_{1}^{2} x_{4}+19.84 x_{1}^{2} x_{3}, \\
\text { s.t. } g_{1}(x)= & -x_{1}+0.0193 x_{3} \leq 0, \\
g_{2}(x)= & -x_{2}+0.00954 x_{3} \leq 0, \\
g_{3}(x)= & -\pi x_{3}^{2} x_{4}-\frac{4}{3} \pi x_{3}^{3}+1,296,000 \leq 0, \\
g_{4}(x)= & x_{4}-240 \leq 0, \\
0 \leq & x_{1}, x_{2} \leq 99, \\
10 \leq & x_{3}, x_{4} \leq 200,
\end{aligned}
$$$$
x_{1} \text { and } x_{2} \text { are integer multiples of } 0.0625 \text {. }
$$ 


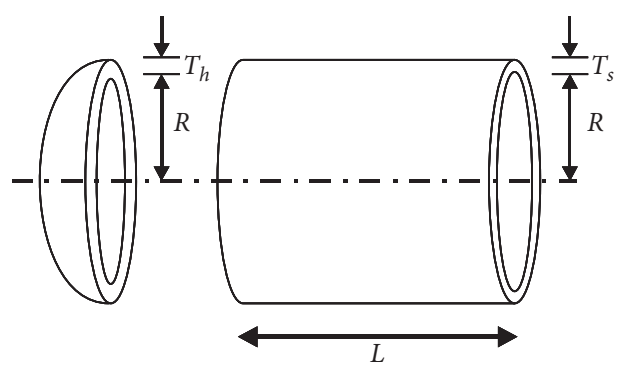

Figure 5: Pressure vessel design problem.

The best, mean, worst, and standard deviation values of the pressure vessel design problem for the 30 runs are shown in Table 28. MPEPSO outperforms SCA, WOA, and BLPSO in terms of "best" and "mean." Meanwhile, in terms of "worst" and "std," MPEPSO also provides promising results better than SCA and WOA, and the differences between MPEPSO and BLPSO are small.

Table 29 shows the best solutions of MPEPSO, SCA, WOA, and BLPSO. From Table 29, we can find that the best feasible solution provided by MPEPSO is better than other algorithms.

4.5.3. Cantilever Beam Design Problem. The cantilever beam shown is built from five elements with cross section [63]. The purpose of the cantilever beam design problem is to minimize the weight of the beam. The design variables are the heights $x_{j}$ of the five beam elements. The final formulation for the cantilever beam design problem is shown as follows:

$$
\begin{aligned}
& \min f(x)=0.0624\left(x_{1}+x_{2}+x_{3}+x_{4}+x_{5}\right), \\
& \text { s.t. } g(x)=\frac{61}{x_{1}^{3}}+\frac{37}{x_{2}^{3}}+\frac{19}{x_{3}^{3}}+\frac{7}{x_{4}^{3}}+\frac{1}{x_{5}^{3}}-1 \leq 0, \\
& 0.01 \leq x_{1}, x_{2}, x_{3}, x_{4}, x_{5} \leq 100 .
\end{aligned}
$$

The best, mean, worst, and standard deviation values of the cantilever beam design problem for the 30 runs are shown in Table 30. From this table, we can find that MPEPSO outperforms SCA, WOA, and BLPSO in terms of all comparison aspects.

Table 31 shows the best solutions of MPEPSO, SCA, WOA, and BLPSO. From Table 31, we can find that the best feasible solution provided by MPEPSO is better than other algorithms.

4.5.4. Gear Train Design Problem. The purpose of the gear train design problem is to design a gear train that the gear ratio should be close to $1 / 6.931$ [61]. The design variables are the number of teeth on four gears, $x_{1}, x_{2}, x_{3}$, and $x_{4}$. Since the number of teeth should be integers, this is an integer programming problem and the final formulation for the gear train design problem is shown as follows:
TABle 28: Computational results of the pressure vessel design problem.

\begin{tabular}{|c|c|c|c|c|c|}
\hline \multirow{2}{*}{ Algorithms } & \multicolumn{4}{|c|}{ Optimal decision vector } & \multirow{2}{*}{$\begin{array}{l}\text { Optimum } \\
\text { value }\end{array}$} \\
\hline & $x_{1}$ & $x_{2}$ & $x_{3}$ & $x_{4}$ & \\
\hline MPEPSO & 0.8125 & 0.4375 & 42.09845 & 176.6366 & 6059.714 \\
\hline SCA & 0.8125 & 0.4375 & 41.64538 & 188.6181 & 6261.169 \\
\hline WOA & 0.8125 & 0.4375 & 41.33188 & 186.3729 & 6155.302 \\
\hline BLPSO & 0.8125 & 0.4375 & 42.08892 & 176.7547 & 6060.876 \\
\hline
\end{tabular}

\begin{tabular}{lcccc}
\hline Algorithms & Best & Mean & Worst & Std \\
\hline MPEPSO & $\mathbf{6 0 5 9 . 7 1 4}$ & $\mathbf{6 2 5 1 . 2 1 8}$ & 6820.41 & 219.6223 \\
SCA & 6261.169 & 7266.512 & 8825.87 & 672.9376 \\
WOA & 6155.302 & 8486.523 & 14148.37 & 1747.194 \\
BLPSO & 6060.876 & 6270.428 & $\mathbf{6 7 7 5 . 8 0 6}$ & $\mathbf{2 0 5 . 8 8 8 8}$ \\
\hline
\end{tabular}

TABLE 29: Best results of the pressure vessel design problem.

$$
\begin{aligned}
& \min f(x)=\left(\frac{1}{6.931}-\frac{x_{2} x_{3}}{x_{1} x_{4}}\right)^{2}, \\
& \text { s.t. } 12 \leq x_{i} \leq 60, x_{i} \in Z^{+}, \forall i .
\end{aligned}
$$

The best, mean, worst, and standard deviation values of the cantilever beam design problem for the 30 runs are shown in Table 32. From this table, we can find that MPEPSO and BLPSO provide the best results in terms of "best," while MPEPSO outperforms SCA, WOA, and BLPSO in terms of "mean," "worst," and "std."

Table 33 shows the best solutions of MPEPSO, SCA, WOA, and BLPSO. From Table 33, we can find that MPEPSO and BLPSO provide the best feasible solutions.

4.6. Discussion. The performance of MPEPSO is evaluated by different types of problems including unimodal, simple multimodal, hybrid, and composition functions. The computational results of MPEPSO are compared with its component algorithms and advanced PSO algorithms. MPEPSO performs consistently well on both 10-dimensional and 30-dimensional problems of the CEC 2014 test suite.

Firstly, the computational results show that MPEPSO performs better than its component algorithms, LDWPSO, UPSO, and CLPSO. These results suggest that the multipopulation ensemble approach in PSO effectively allocates the reward subpopulation to the good performed strategy. Therefore, a good performed strategy can have more computational expense to find better solutions.

Secondly, MPEPSO is also compared with three advanced PSO variants developed by different methodologies. DMS-PSO-HS hybridizes PSO with a harmony search, LIPS introduces a new topology structure, and SL-PSO introduces a new searching strategy. The results indicate that MPEPSO can not only outperform its component algorithms, but also 
TABLE 30: Computational results of the cantilever beam design problem.

\begin{tabular}{lcccc}
\hline Algorithms & Best & Mean & Worst & Std \\
\hline MPEPSO & $\mathbf{1 . 3 3 9 9 5 6}$ & $\mathbf{1 . 3 3 9 9 5 8}$ & $\mathbf{1 . 3 3 9 9 6 1}$ & $\mathbf{9 . 9 2 E - 0 7}$ \\
SCA & 1.356647 & 1.382846 & 1.426982 & 0.017955 \\
WOA & 1.347944 & 1.436524 & 1.664954 & 0.076076 \\
BLPSO & 1.339957 & 1.339959 & 1.339965 & $2.11 E-06$ \\
\hline
\end{tabular}

TABLE 31: Best results of the cantilever beam design problem.

\begin{tabular}{lccccc}
\hline \multirow{2}{*}{ Algorithms } & \multicolumn{4}{c}{ Optimal decision vector } & Optimum \\
& $x_{1}$ & $x_{2}$ & $x_{3}$ & $x_{4}$ & value \\
\hline MPEPSO & 6.01548 & 5.308041 & 4.495519 & 3.502155 & $\mathbf{1 . 3 3 9 9 5 6}$ \\
SCA & 6.701277 & 5.313045 & 4.37163 & 3.091864 & 1.356647 \\
WOA & 5.648277 & 5.78434 & 4.452996 & 3.501799 & 1.347944 \\
BLPSO & 6.014017 & 5.30808 & 4.496492 & 3.500623 & 1.339957 \\
\hline
\end{tabular}

TABLE 32: Computational results of the gear train design problem.

\begin{tabular}{lcccc}
\hline Algorithms & Best & Mean & Worst & Std \\
\hline MPEPSO & $\mathbf{2 . 7 0 E - 1 2}$ & $\mathbf{2 . 5 9 E - \mathbf { 0 7 }}$ & $\mathbf{7 . 7 3 E - \mathbf { 0 6 }}$ & $\mathbf{1 . 4 1 E - \mathbf { 0 6 }}$ \\
SCA & $2.31 E-11$ & $1.89 E-06$ & $2.65 E-05$ & $5.82 E-06$ \\
WOA & $1.55 E-10$ & $1.57 E-05$ & $8.24 E-05$ & $2.25 E-05$ \\
BLPSO & $\mathbf{2 . 7 0 E - 1 2}$ & $4.91 E-06$ & $2.64 E-05$ & $7.89 E-06$ \\
\hline
\end{tabular}

TABle 33: Best results of the gear train design problem.

\begin{tabular}{llllll}
\hline \multirow{2}{*}{ Algorithms } & \multicolumn{5}{c}{ Optimal decision vector } \\
& $x_{1}$ & $x_{2}$ & $x_{3}$ & $x_{4}$ & Optimum value \\
\hline MPEPSO & 49 & 19 & 16 & 43 & $\mathbf{2 . 7 0 E - 1 2}$ \\
SCA & 51 & 15 & 26 & 53 & $2.31 E-11$ \\
WOA & 43 & 21 & 13 & 44 & $1.55 E-10$ \\
BLPSO & 49 & 16 & 19 & 43 & $\mathbf{2 . 7 0 E}-\mathbf{1 2}$ \\
\hline
\end{tabular}

the advanced PSO variants. Moreover, the performance of MPEPSO is compared with that of five other metaheuristic algorithms. According to [52], FA and CS have a good performance on the CEC 2014 test suite comparing with other nature-inspired algorithms. SCA and GCMBO are the most recently proposed algorithms. MPEPSO has better performance than FA, CS, SCA, and GCMBO. MPEPSO is not competitive compared with L-SHADE, the winner of the CEC 2014 competition. However, the objective of this paper is not to provide the most efficient algorithm for CEC 2014. We found that the proposed MPEPSO outperforms the component PSO algorithms. The results suggest that the multipopulation ensemble approach is a promising method, and this approach has been successfully applied to develop an efficient PSO algorithm. It significantly contributes to the development of efficient PSO algorithms. In the future, it is possible to explore the ensemble of the top performed algorithms on CEC 2014, such as L-SHADE, by the multipopulation ensemble approach.

Thirdly, the computational complexity tests show that MPEPSO uses competitive and reasonable computation costs. The nature of MPEPSO is the dynamical combination of LDWPSO, and UPSO and CLPSO. In the tests, UPSO and CLPSO have higher computational costs compared with MPEPSO, while LDWPSO has relatively lower computational costs. MPEPSO ranks 3 in the computational complexity tests on both 10 and 30 dimensions. That means the multipopulation ensemble approach in MPEPSO is not time-consuming.

Also, MPEPSO is applied to solve four real-world engineering design problems including the tension/compression spring design problem, the pressure vessel design problem, the cantilever beam design problem, and the gear train design problem. The performance of MPEPSO is compared with two recently proposed metaheuristic algorithms, SCA and WOA, and one recently proposed PSO variant, BLPSO. The results suggest that MPEPSO can obtain the best results on all the engineering design problems comparing with SCA, WOA, and BLSPO. Therefore, we confirmed the advantages of MPEPSO on solving real-world engineering design problems.

Overall, the experimental results show the advantages of the proposed algorithm, and MPEPSO is a promising algorithm.

\section{Conclusion}

In this paper, a multipopulation ensemble particle swarm optimizer (MPEPSO) is proposed to realize the ensemble of three efficient and simple PSO searching strategies, LDWPSO, UPSO, and CLPSO. The indicator subpopulations have a relatively small number of particles compared with the reward subpopulation. At the end of a learning period, the performance of each strategy is evaluated to select the best-performed strategy, and then the reward subpopulation is allocated to the corresponding strategy. By dynamically allocating the reward subpopulation to the good performed strategy at different learning periods, the appropriate PSO searching strategy will have more particles to find a better solution. Therefore, the performance of MPEPSO is enhanced. The performance of MPEPSO on the CEC 2014 test suite is compared with that of other PSO variants. The computational results show that the proposed MPEPSO can outperform the other PSO variants on these benchmark functions for both 10-dimensional and 30-dimensional problems. Furthermore, MPEPSO is applied to solve four real-world engineering design problems. MPEPSO provides better results on these problems compared with several other algorithms.

There are several future research directions. First, we realized the ensemble of three simple PSO searching strategies in this paper. The proposed algorithm is still outperformed by L-SHADE on the CEC 2014 test suite. Further research might explore the ensemble of the top performed algorithms, such as L-SHADE [50], jSO [64], and CoBiDE [65], by the multipopulation ensemble approach. Second, a limited number of PSO strategies are incorporated in the proposed algorithm. A further study could investigate the effects of using more PSO variants [66-68]. 


\author{
Abbreviations \\ PSO: $\quad$ Particle swarm optimization \\ MPEPSO: Multipopulation ensemble PSO \\ LDWPSO: Linearly decreasing inertia weight PSO \\ CLPSO: Comprehensive learning PSO \\ UPSO: Unified PSO \\ HPSO: Heterogeneous PSO \\ SL-PSO: Self-adaptive learning-based PSO \\ HCLPSO: Heterogeneous comprehensive learning PSO \\ EPSO: $\quad$ Ensemble PSO \\ DMS-PSO- Dynamic multiswarm PSO with harmony \\ HS: $\quad$ search \\ LIPS: Distance-based locally informed PSO \\ SL-PSO: Social learning PSO \\ DE: Differential evolution \\ CS: $\quad$ Cuckoo search \\ FA: $\quad$ Firefly algorithm \\ GCMBO: Monarch butterfly optimization with greedy \\ strategy and crossover operator \\ L-SHADE: Success-history-based adaptive DE with linear \\ population size reduction \\ SCA: $\quad$ Sine cosine algorithm \\ WOA: Whale optimization algorithm \\ BLPSO: Biogeography-based learning particle swarm \\ optimization.
}

\section{Notation Indices}

$i$ : Index of particles

$d$ : Index of dimension

$h$ : Index of the PSO searching strategy.

\section{Parameters}

$V_{i}^{d}: \quad$ Velocity of the $i$ th particle on the $d$ th dimension

$X_{i}^{d}$ : $\quad$ Position of the $i$ th particle on the $d$ th dimension

$N$ : $\quad$ Total population size

D: $\quad$ Dimension number of the problem

$\omega: \quad$ Inertia factor

$c: \quad$ Accelerate coefficients

pbest $_{i}$ : Previous best position of the $i$ th particle

gbest: Global best position of all the particle

rand: Random numbers in the interval of $[0,1]$

$G_{i}^{d}$ : $\quad$ Velocity of particle $i$ in the global version PSO

$L_{i}^{d}$ : $\quad$ Velocity of particle $i$ in the local version PSO

$\mathscr{X}$ : $\quad$ Constriction factor

$n$ best $_{i}$ : Personal best position of the best neighborhood of $X_{i}$

$U_{i}^{d}$ : $\quad$ Velocity of the $i$ th particle on the $d$ th dimension in UPSO

$P c_{i}: \quad$ Learning probability

$\operatorname{pop}_{h}$ : $\quad$ Set of indicator subpopulation $h$

pop $_{r}$ : Set of reward subpopulation

$N_{h}$ : $\quad$ Population size of the indicator subpopulation $h$

$N_{r}$ : Population size of the reward subpopulation

$\lambda_{h}$ : $\quad$ Proportion of pop $_{h}$ in the population

$\Delta f_{h}: \quad$ Strategy improvement

LP: $\quad$ Learning period iter: Number of iterations

FEs: $\quad$ Number of function evaluations

MaxFEs: Maximum number of function evaluations.

\section{Data Availability}

The source codes are available at https://github.com/zi-angliu/MPEPSO. The data used to support the findings of this study are available from the corresponding author upon request.

\section{Conflicts of Interest}

The authors declare no conflicts of interest.

\section{Acknowledgments}

This research was conducted by the aid of funding provided by JSPS Grant-in-Aid for Challenging Exploratory Research 17K18951.

\section{References}

[1] J. Kennedy and R. Eberhart, "Particle swarm optimization," in Proceedings of the ICNN'95-International Conference on Neural Networks, pp. 1942-1948, Perth, WA, Australia, November 1995.

[2] D. Wang, D. Tan, and L. Liu, "Particle swarm optimization algorithm: an overview," Soft Computing, vol. 22, no. 2, pp. 387-408, 2018.

[3] Q. He and L. Wang, "An effective co-evolutionary particle swarm optimization for constrained engineering design problems," Engineering Applications of Artificial Intelligence, vol. 20, no. 1, pp. 89-99, 2007.

[4] X. Zhao, Y. Zhou, and Y. Xiang, "A grouping particle swarm optimizer," Applied Intelligence, vol. 49, no. 8, pp. 2862-2873, 2019.

[5] N. Ben Guedria, "Improved accelerated PSO algorithm for mechanical engineering optimization problems," Applied Soft Computing, vol. 40, pp. 455-467, 2016.

[6] H. Garg, "A hybrid PSO-GA algorithm for constrained optimization problems," Applied Mathematics and Computation, vol. 274, pp. 292-305, 2016.

[7] Y. Zhang, D. Gong, Y. Hu, and W. Zhang, "Feature selection algorithm based on bare bones particle swarm optimization," Neurocomputing, vol. 148, pp. 150-157, 2015.

[8] Y. Zhang, D.-w. Gong, and J. Cheng, "Multi-objective particle swarm optimization approach for cost-based feature selection in classification," IEEE/ACM Transactions on Computational Biology and Bioinformatics, vol. 14, no. 1, pp. 64-75, 2017.

[9] M. A. Tawhid and K. B. Dsouza, "Hybrid binary bat enhanced particle swarm optimization algorithm for solving feature selection problems," Applied Computing and Informatics, vol. 16, no. 1-2, pp. 117-136, 2020.

[10] B. Liu, L. Wang, and Y.-H. Jin, "An effective hybrid particle swarm optimization for no-wait flow shop scheduling," The International Journal of Advanced Manufacturing Technology, vol. 31, no. 9-10, pp. 1001-1011, 2007.

[11] F. Zhao, S. Qin, G. Yang, W. Ma, C. Zhang, and H. Song, “A factorial based particle swarm optimization with a population adaptation mechanism for the no-wait flow shop scheduling problem with the makespan objective," Expert Systems with Applications, vol. 126, pp. 41-53, 2019. 
[12] H. Zhou, J. Pang, P.-K. Chen, and F.-D. Chou, "A modified particle swarm optimization algorithm for a batch-processing machine scheduling problem with arbitrary release times and non-identical job sizes," Computers \& Industrial Engineering, vol. 123, pp. 67-81, 2018.

[13] Y.-W. Chen, L.-C. Wang, A. Wang, and T.-L. Chen, "A particle swarm approach for optimizing a multi-stage closed loop supply chain for the solar cell industry," Robotics and Computer-Integrated Manufacturing, vol. 43, pp. 111-123, 2017.

[14] K. Patne, N. Shukla, S. Kiridena, and M. K. Tiwari, "Solving closed-loop supply chain problems using game theoretic particle swarm optimisation," International Journal of Production Research, vol. 56, no. 17, pp. 5836-5853, 2018.

[15] H. Soleimani and G. Kannan, "A hybrid particle swarm optimization and genetic algorithm for closed-loop supply chain network design in large-scale networks," Applied Mathematical Modelling, vol. 39, no. 14, pp. 3990-4012, 2015.

[16] Y. Shi and R. C. Eberhart, "Empirical study of particle swarm optimization," in Proceedings of the 1999 Congress on Evolutionary Computation-CEC99 (Cat. No. 99TH8406), pp. 1945-1950, Washington, DC, USA, July 1999.

[17] W. Dong and M. Zhou, "A supervised learning and control method to improve particle swarm optimization algorithms," IEEE Transactions on Systems, Man, and Cybernetics: Systems, vol. 47, no. 7, pp. 1135-1148, 2017.

[18] Y.-J. Zheng, H.-F. Ling, and Q. Guan, “Adaptive parameters for a modified comprehensive learning particle swarm optimizer," Mathematical Problems in Engineering, vol. 2012, Article ID 207318, 11 pages, 2012.

[19] J. Kennedy, "Bare bones particle swarms," in Proceedings of the 2003 IEEE Swarm Intelligence Symposium. SIS'03 (Cat. No.03EX706), pp. 80-87, Indianapolis, IN, USA, April 2003.

[20] J. J. Liang, A. K. Qin, P. N. Suganthan, and S. Baskar, "Comprehensive learning particle swarm optimizer for global optimization of multimodal functions," IEEE Transactions on Evolutionary Computation, vol. 10, no. 3, pp. 281-295, 2006.

[21] P.-Y. Yang, F.-I. Chou, J.-T. Tsai, and J.-H. Chou, "Adaptiveuniform-experimental-design-based fractional-order particle swarm optimizer with non-linear time-varying evolution," Applied Sciences, vol. 9, no. 24, p. 5537, 2019.

[22] R. Cheng and Y. Jin, "A competitive swarm optimizer for large scale optimization," IEEE Transactions on Cybernetics, vol. 45, no. 2, pp. 191-204, 2015.

[23] J. Kennedy and R. Mendes, "Population structure and particle swarm performance," in Proceedings of the 2002 Congress on Evolutionary Computation CEC'02 (Cat. No.02TH8600), pp. 1671-1676, Honolulu, HI, USA, May 2002.

[24] W. Fang, J. Sun, H. Chen, and X. Wu, "A decentralized quantum-inspired particle swarm optimization algorithm with cellular structured population," Information Sciences, vol. 330, pp. 19-48, 2016.

[25] Y.-J. Gong, J.-J. Li, Y. Zhou et al., "Genetic learning particle swarm optimization," IEEE Transactions on Cybernetics, vol. 46, no. 10, pp. 2277-2290, 2016.

[26] S.-Z. Zhao, P. N. Suganthan, Q.-K. Pan, and M. Fatih Tasgetiren, "Dynamic multi-swarm particle swarm optimizer with harmony search," Expert Systems with Applications, vol. 38, no. 4, pp. 3735-3742, 2011.

[27] X. Chen, H. Tianfield, C. Mei, W. Du, and G. Liu, "Biogeography-based learning particle swarm optimization," Soft Computing, vol. 21, no. 24, pp. 7519-7541, 2017.

[28] H.-b. Ouyang, L.-q. Gao, X.-y. Kong, S. Li, and D.-x. Zou, "Hybrid harmony search particle swarm optimization with global dimension selection," Information Sciences, vol. 346347, pp. 318-337, 2016.

[29] Y. Wang, B. Li, T. Weise, J. Wang, B. Yuan, and Q. Tian, "Selfadaptive learning based particle swarm optimization," Information Sciences, vol. 181, no. 20, pp. 4515-4538, 2011.

[30] A. Ratnaweera, S. K. Halgamuge, and H. C. Watson, "Selforganizing hierarchical particle swarm optimizer with timevarying acceleration coefficients," IEEE Transactions on Evolutionary Computation, vol. 8, no. 3, pp. 240-255, 2004.

[31] B. Y. Qu, P. N. Suganthan, and S. Das, "A distance-based locally informed particle swarm model for multimodal optimization," IEEE Transactions on Evolutionary Computation, vol. 17, no. 3, pp. 387-402, 2013.

[32] A. P. Engelbrecht, "Heterogeneous particle swarm optimization," in Swarm Intelligence, Springer Berlin Heidelberg, Berlin, Heidelberg, Germany, 2010.

[33] N. Lynn and P. N. Suganthan, "Heterogeneous comprehensive learning particle swarm optimization with enhanced exploration and exploitation," Swarm and Evolutionary Computation, vol. 24, pp. 11-24, 2015.

[34] N. Lynn and P. N. Suganthan, "Ensemble particle swarm optimizer," Applied Soft Computing, vol. 55, pp. 533-548, 2017.

[35] B. K. Kannan and S. N. Kramer, “An augmented Lagrange multiplier based method for mixed integer discrete continuous optimization and its applications to mechanical design," Journal of Mechanical Design, vol. 116, no. 2, pp. 405-411, 1994.

[36] K. E. Parsopoulos and M. N. Vrahatis, "UPSO: a unified particle swarm optimization scheme," in International Conference of Computational Methods in Sciences and Engineering 2004 (ICCMSE 2004), T. Simos and G. Maroulis, Eds., CRC Press, London, UK, 1st edition, 2019.

[37] G. Wu, R. Mallipeddi, P. N. Suganthan, R. Wang, and H. Chen, "Differential evolution with multi-population based ensemble of mutation strategies," Information Sciences, vol. 329, pp. 329-345, 2016.

[38] G. Wu, X. Shen, H. Li, H. Chen, A. Lin, and P. N. Suganthan, "Ensemble of differential evolution variants," Information Sciences, vol. 423, pp. 172-186, 2018.

[39] G. Wu, R. Mallipeddi, and P. N. Suganthan, "Ensemble strategies for population-based optimization algorithms - a survey," Swarm and Evolutionary Computation, vol. 44, pp. 695-711, 2019.

[40] R. C. Eberhart and Y. Shi, "Comparing inertia weights and constriction factors in particle swarm optimization," in Proceedings of the 2000 Congress on Evolutionary Computation CECO0 (Cat. No.00TH8512), La Jolla, CA, USA, pp. 84-88, July 2000, https://doi.org/10.1109/CEC.2000.870279.

[41] J. J. Liang, B. Y. Qu, and P. N. Suganthan, "Problem definitions and evaluation criteria for the CEC 2014 special session and competition on single objective real-parameter numerical optimization," Technical Report, Computational Intelligence Laboratory; Nanyang Technological University, Zhengzhou University, Zhengzhou, China; Singapore, 2013.

[42] P. N. Suganthan, Codes for CEC 2014 Test Suite, 2020, https:// github.com/P-N-Suganthan/CEC2014.

[43] R. Cheng and Y. Jin, "A social learning particle swarm optimization algorithm for scalable optimization," Information Sciences, vol. 291, pp. 43-60, 2015.

[44] J. J. Liang and P. N. Suganthan, "Dynamic multi-swarm particle swarm optimizer," in Proceedings of the 2005 IEEE Swarm Intelligence Symposium (SIS 2005), pp. 124-129, Pasadena, CA, USA, June 2005. 
[45] Z. W. Geem, J. H. Kim, and G. V. Loganathan, "A new heuristic optimization algorithm: harmony search," Simulation, vol. 76, pp. 60-68, 2001.

[46] X.-S. Yang, "Suash Deb, cuckoo search via Lévy flights," in Proceedings of the 2009 World Congress on Nature \& Biologically Inspired Computing (NaBIC), pp. 210-214, Coimbatore, India, December 2009.

[47] X.-S. Yang, Nature-Inspired Optimization Algorithms, Elsevier, London, UK, 1st edition, 2014.

[48] S. Mirjalili, "SCA: a Sine Cosine Algorithm for solving optimization problems," Knowledge-Based Systems, vol. 96, pp. 120-133, 2016.

[49] G.-G. Wang, S. Deb, X. Zhao, and Z. Cui, "A new monarch butterfly optimization with an improved crossover operator," Operational Research, vol. 18, no. 3, pp. 731-755, 2018.

[50] R. Tanabe and A. S. Fukunaga, "Improving the search performance of SHADE using linear population size reduction," in Proceedings of the 2014 IEEE Congress on Evolutionary Computation (CEC), pp. 1658-1665, Beijing, China, July 2014.

[51] A. Zamuda and J. D. H. Sosa, "Success history applied to expert system for underwater glider path planning using differential evolution," Expert Systems with Applications, vol. 119, pp. 155-170, 2019.

[52] P. Bujok, J. Tvrdík, and R. Poláková, “Comparison of natureinspired population-based algorithms on continuous optimisation problems," Swarm and Evolutionary Computation, vol. 50, Article ID 100490, 2019.

[53] G. Singh, K. Deep, and A. K. Nagar, "Cell-like P-systems based on rules of particle swarm optimization," Applied Mathematics and Computation, vol. 246, pp. 546-560, 2014.

[54] Q. Liu, L. Wu, W. Xiao, F. Wang, and L. Zhang, "A novel hybrid bat algorithm for solving continuous optimization problems," Applied Soft Computing, vol. 73, pp. 67-82, 2018.

[55] S. Družeta and S. Ivić, "Examination of benefits of personal fitness improvement dependent inertia for Particle Swarm Optimization," Soft Computing, vol. 21, pp. 3387-3400, 2017.

[56] S. Mirjalili and A. Lewis, "The whale optimization algorithm," Advances in Engineering Software, vol. 95, pp. 51-67, 2016.

[57] D. Simon, "Biogeography-based optimization," IEEE Transactions on Evolutionary Computation, vol. 12, no. 6, pp. 702-713, 2008.

[58] J. S. Arora, "Optimum design problem formulation," in Introduction to Optimum Design, Elsevier/Academic Press, Boston, MA, USA, 2nd edition, 2004.

[59] O. Camarena, E. Cuevas, M. Pérez-Cisneros, F. Fausto, A. González, and A. Valdivia, "Ls-II: an improved locust search algorithm for solving optimization problems," Mathematical Problems in Engineering, vol. 2018, Article ID 4148975, 15 pages, 2018.

[60] X. S. Yang and S. Deb, "Engineering optimisation by Cuckoo search," International Journal of Mathematical Modelling and Numerical Optimisation, vol. 1, no. 4, pp. 330-343, 2010.

[61] E. Sandgren, "Nonlinear integer and discrete programming in mechanical design optimization," Journal of Mechanical Design, vol. 112, no. 2, pp. 223-229, 1990.

[62] M. Luqman, M. Saeed, J. Ali, and M. F. Tabassum, "Radial artificial bee colony algorithm for constraint engineering problems," Pakistan Journal of Science, vol. 69, p. 10, 2017.

[63] K. Svanberg, "The method of moving asymptotes-a new method for structural optimization," International Journal for Numerical Methods in Engineering, vol. 24, no. 2, pp. 359-373, 1987.

[64] J. Brest, M. S. Maucec, and B. Boskovic, "Single objective realparameter optimization: algorithm jSO," in Proceedings of the
2017 IEEE Congress on Evolutionary Computation (CEC), pp. 1311-1318, Donostia-San Sebastián, Spain, June 2017.

[65] Y. Wang, H.-X. Li, T. Huang, and L. Li, "Differential evolution based on covariance matrix learning and bimodal distribution parameter setting," Applied Soft Computing, vol. 18, pp. 232-247, 2014.

[66] L. dos Santos Coelho and V. C. Mariani, "Particle swarm approach based on quantum mechanics and harmonic oscillator potential well for economic load dispatch with valvepoint effects," Energy Conversion and Management, vol. 49, no. 11 , pp. $3080-3085,2008$.

[67] L. d. S. Coelho and V. C. Mariani, "A novel chaotic particle swarm optimization approach using Hénon map and implicit filtering local search for economic load dispatch," Chaos, Solitons \& Fractals, vol. 39, no. 2, pp. 510-518, 2009.

[68] V. C. Mariani, A. R. K. Duck, F. A. Guerra, L. d. S. Coelho, and R. V. Rao, "A chaotic quantum-behaved particle swarm approach applied to optimization of heat exchangers," Applied Thermal Engineering, vol. 42, pp. 119-128, 2012. 